\title{
One-pot access to a library of dispirooxindole-pyrrolidine/pyrrolothiazole- thiochromane hybrids via three-component 1,3-dipolar cycloaddition reactions
}

Gandhi Uma Rani ${ }^{\mathrm{a}}$, Sundaravel Vivek Kumar ${ }^{\mathrm{a}}$, Chelliah Bharkavi, J. Carlos Menéndez ${ }^{\mathrm{b}}$ and

$$
\text { Subbu Perumal }{ }^{\text {a* }}
$$

${ }^{a}$ Department of Organic Chemistry, School of Chemistry, Madurai Kamaraj University, Madurai-625 021, Tamil Nadu, India

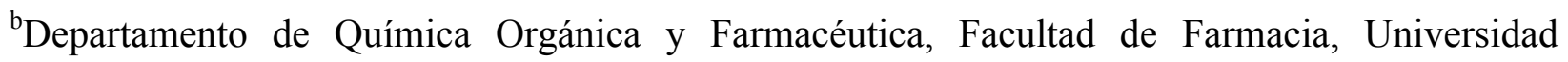
Complutense, 28040 Madrid, Spain

\section{Experimental Section}

\subsection{General methods}

The melting points were measured in open capillary tubes and are uncorrected. The ${ }^{1} \mathrm{H}$ NMR, ${ }^{13} \mathrm{C}$ NMR, DEPT, H,H-COSY and HMBC spectra were recorded on a Bruker (Avance) $300 \mathrm{MHz}$ NMR instrument using TMS as internal standard and $\mathrm{CDCl}_{3}$ as solvent. Standard Bruker software was used throughout. Chemical shifts are given in parts per million ( $\delta$-scale) and the coupling constants are given in Hertz. Silica gel-G plates (Merck) were used for TLC analysis with a mixture of petroleum ether $\left(60-80{ }^{\circ} \mathrm{C}\right)$ and ethyl acetate as eluent. Mass spectra were recorded in LCQ Fleet mass spectrometer, Thermo Fisher Instruments Limited, US. Electrospray ionisation mass spectrometry (ESI-MS) analysis was performed in the positive/negative ion mode on a liquid chromatography ion trap. Combustion microanalyses were performed by the CAI de Microanálisis Elemental, Universidad Complutense, using a Leco-932 CHNS microanalyzer. 
General procedure for the synthesis of dispirooxindole-pyrrolidine/ pyrrolothiazolethiochroman-4-one heterocyclic hybrids:

A mixture of 3-arylidenethiocroman-4-one $(1 \mathrm{mmol})$, isatin $(1 \mathrm{mmol})$ and sarcosine/ Lthioproline $(1 \mathrm{mmol})$ in $\mathrm{MeOH}(5 \mathrm{ml})$ was heated under reflux for $5-6 \mathrm{~h}$. After completion of the reaction as evident from TLC, the solvent was removed and the crude product was purified by column chromatography using petroleum ether-ethyl acetate mixture $(4: 1 \mathrm{v} / \mathrm{v})$ to afford the desired products.

\section{4'-(4-Fluorophenyl)-1'-methyldispiro[indoline-3,2'-pyrrolidine-3',3"'-thiochromane]-2,4"'-}

dione 4\{1,1,1\}: White solid. Yield: 82\%; mp (petroleum ether-ethyl acetate 1:4 v/v) =156-157 ${ }^{\circ} \mathrm{C}$; IR (KBr) v $v_{\max }: 3209,3063,2954,2845,1696,1672,1617,1587,1511,1470 \mathrm{~cm}^{-1} ;{ }^{1} \mathrm{H}$ NMR $\left(300 \mathrm{MHz}, \mathrm{CDCl}_{3}\right) \delta_{\mathrm{H}}: 2.09(\mathrm{~s}, 3 \mathrm{H}), 2.59(\mathrm{~d}, J=14.4 \mathrm{~Hz}, 1 \mathrm{H}), 3.33(\mathrm{~d}, J=14.4 \mathrm{~Hz}, 1 \mathrm{H}), 3.44$ (t, $J=8.4 \mathrm{~Hz}, 1 \mathrm{H}), 3.92(\mathrm{t}, J=9.9 \mathrm{~Hz}, 1 \mathrm{H}), 5.16(\mathrm{dd}, J=10.5,7.8 \mathrm{~Hz}, 1 \mathrm{H}), 6.57(\mathrm{t}, J=7.5 \mathrm{~Hz}, 1 \mathrm{H})$, $6.65(\mathrm{~d}, J=7.8 \mathrm{~Hz}, 1 \mathrm{H}), 6.75(\mathrm{t}, J=6.4 \mathrm{~Hz}, 2 \mathrm{H}), 6.95(\mathrm{~d}, J=7.5 \mathrm{~Hz}, 1 \mathrm{H}), 7.00(\mathrm{~d}, J=8.7 \mathrm{~Hz}$, 2H), 7.04-7.09 (m, 2H), 7.49 (t, $J=6.6 \mathrm{~Hz}, 2 \mathrm{H}), 7.69$ (br s, 1H), 8.16 (dd, $J=8.1,1.5 \mathrm{~Hz}, 1 \mathrm{H})$; ${ }^{13} \mathrm{C}$ NMR $\left(75 \mathrm{MHz}, \mathrm{CDCl}_{3}\right) \delta_{\mathrm{C}}: 34.0,34.1,46.5,56.9,59.9,74.9,109.3,115.3\left({ }^{2} J_{\mathrm{C}, \mathrm{F}}=20.9 \mathrm{~Hz}\right)$, $122.0,124.5,126.0,126.3,127.2,129.0,130.7,130.9,132.1\left({ }^{3} J_{\mathrm{C}, \mathrm{F}}=7.9 \mathrm{~Hz}\right), 132.3,133.4$, 141.6, 142.0, $162.0\left({ }^{1} J_{\mathrm{C}, \mathrm{F}}=244.3 \mathrm{~Hz}\right), 177.1,193.5$; ESI-MS: m/z. Calcd: 444.13; Found: 445.13(M+1); Anal. Calcd for $\mathrm{C}_{26} \mathrm{H}_{21} \mathrm{FN}_{2} \mathrm{O}_{2} \mathrm{~S}$ : C, 70.25; H, 4.76; N, 6.30; \%. Found C, 69.25; H, $4.69 ; \mathrm{N}, 6.22 \%$.

4'-(4-Chlorophenyl)-1'-methyldispiro[indoline-3,2'-pyrrolidine-3',3'"-thiochromane]-2,4"'-

dione 4\{2,1,1\}: White solid. Yield: 81\%; mp (petroleum ether-ethyl acetate 1:4 v/v) =188-189 ${ }^{\circ} \mathrm{C}$; IR (KBr) v $v_{\max }: 3293,2920,2854,1698,1670,1619,1587,1491 \mathrm{~cm}^{-1} ;{ }^{1} \mathrm{H}$ NMR (300 MHz, $\left.\mathrm{CDCl}_{3}\right) \delta_{\mathrm{H}}: 2.09(\mathrm{~s}, 3 \mathrm{H}), 2.61(\mathrm{~d}, J=14.1 \mathrm{~Hz}, 1 \mathrm{H}), 3.33(\mathrm{~d}, J=14.4 \mathrm{~Hz}, 1 \mathrm{H}), 3.43(\mathrm{t}, J=8.2 \mathrm{~Hz}$, $1 \mathrm{H}), 3.93(\mathrm{dd}, J=10.5,9.3 \mathrm{~Hz}, 1 \mathrm{H}), 5.16(\mathrm{dd}, J=10.5,7.8 \mathrm{~Hz}, 1 \mathrm{H}), 6.58(\mathrm{t}, J=7.5 \mathrm{~Hz}, 1 \mathrm{H})$, $6.68(\mathrm{~d}, J=7.8 \mathrm{~Hz}, 1 \mathrm{H}), 6.74(\mathrm{~d}, J=7.5 \mathrm{~Hz}, 2 \mathrm{H}), 6.94-7.07(\mathrm{~m}, 3 \mathrm{H}), 7.26-7.31(\mathrm{~m}, 2 \mathrm{H}), 7.47$ $(\mathrm{d}, J=8.4 \mathrm{~Hz}, 2 \mathrm{H}), 8.15(\mathrm{dd}, J=6.6,1.5 \mathrm{~Hz}, 1 \mathrm{H}), 8.27$ (brs, $1 \mathrm{H}) ;{ }^{13} \mathrm{C}$ NMR $\left(75 \mathrm{MHz}, \mathrm{CDCl}_{3}\right)$ $\delta_{\mathrm{C}}: 33.9,34.1,46.6,56.7,59.8,75.0,109.4,122.0,124.5,126.0,126.3,127.1,128.6,129.0$, 130.6, 130.8, 131.4, 132.3, 133.0, 136.3, 141.7, 141.9, 177.6, 193.3; ESI-MS: m/z. Calcd: 460.10; Found: $461.12(\mathrm{M}+1)$. 


\section{4'-(4-Bromophenyl)-1'-methyldispiro[indoline-3,2'-pyrrolidine-3',3'-thiochromane]-2,4"'-}

dione 4\{3,1,1\}: Whitte solid. Yield: 84\%; mp (petroleum ether-ethyl acetate 1:4 v/v) = 217-218 ${ }^{\circ} \mathrm{C}$; IR (KBr) v $v_{\max }: 3170,2861,1692,1668,1621,1586,1471 \mathrm{~cm}^{-1} ;{ }^{1} \mathrm{H} \mathrm{NMR}\left(300 \mathrm{MHz}, \mathrm{CDCl}_{3}\right)$ $\delta_{\mathrm{H}}: 2.09(\mathrm{~s}, 3 \mathrm{H}), 2.61(\mathrm{~d}, J=14.1 \mathrm{~Hz}, 1 \mathrm{H}), 3.32(\mathrm{~d}, J=14.4 \mathrm{~Hz}, 1 \mathrm{H}), 3.43(\mathrm{t}, J=8.4 \mathrm{~Hz}, 1 \mathrm{H})$, $3.91(\mathrm{t}, \mathrm{J}=9.7 \mathrm{~Hz}, 1 \mathrm{H}), 5.14(\mathrm{dd}, J=10.5,7.8 \mathrm{~Hz}, 1 \mathrm{H}), 6.57(\mathrm{t}, J=7.6 \mathrm{~Hz}, 1 \mathrm{H}), 6.65(\mathrm{~d}, J=7.8$ $\mathrm{Hz}, 1 \mathrm{H}), 6.75$ (t, $J=7.05 \mathrm{~Hz}, 2 \mathrm{H}), 6.94-7.09$ (m, 3H), 7.40 (d, $J=7.8 \mathrm{~Hz}, 2 \mathrm{H}), 7.45$ (d, $J=8.4$ $\mathrm{Hz}, 2 \mathrm{H}), 7.79$ (br s, $1 \mathrm{H}), 8.16(\mathrm{~d}, J=7.8 \mathrm{~Hz}, 1 \mathrm{H}) ;{ }^{13} \mathrm{C} \mathrm{NMR}\left(75 \mathrm{MHz}, \mathrm{CDCl}_{3}\right) \delta_{\mathrm{C}}: 34.0,34.1$, 46.7, 56.7, 59.9, 74.9, 109.3, 121.2, 122.0, 124.5, 126.0, 126.3, 127.2, 129.1, 130.6, 130.8, 130.9, 131.6, 132.3, 136.8, 141.6, 142.0, 177.2, 193.3; ESI-MS: m/z. Calcd: 504.05; Found: 505.07 $(\mathrm{M}+1), 507.11(\mathrm{M}+3)$; Anal. Calcd for $\mathrm{C}_{26} \mathrm{H}_{21} \mathrm{BrN}_{2} \mathrm{O}_{2} \mathrm{~S} \mathrm{C}, 61.79 ; \mathrm{H}, 4.19 ; \mathrm{N}, 5.54 ; \%$. Found $\mathrm{C}$, $61.68 ; \mathrm{H}, 4.34 ; \mathrm{N}, 5.50 \%$.

\section{1'-Methyl-4'-phenyldispiro[indoline-3,2'-pyrrolidine-3',3'-thiochromane]-2,4' '-dione}

4\{4,1,1\}: White solid. Yield: 79\%; mp (petroleum ether-ethyl acetate 1:4 v/v) $=214-213{ }^{\circ} \mathrm{C}$; IR (KBr) $v_{\max }: 3202,2862,1692,1670,1587,1471 \mathrm{~cm}^{-1} ;{ }^{1} \mathrm{H}$ NMR $\left(300 \mathrm{MHz}, \mathrm{CDCl}_{3}\right) \delta_{\mathrm{H}}: 2.11(\mathrm{~s}$, $3 \mathrm{H}), 2.62(\mathrm{~d}, J=14.1 \mathrm{~Hz}, 1 \mathrm{H}), 3.37-3.47(\mathrm{~m}, 2 \mathrm{H}), 4.01(\mathrm{t}, J=9.9 \mathrm{~Hz}, 1 \mathrm{H}), 5.20(\mathrm{dd}, J=10.6$, $7.9 \mathrm{~Hz}, 1 \mathrm{H}), 6.58(\mathrm{t}, J=7.6 \mathrm{~Hz}, 1 \mathrm{H}), 6.68(\mathrm{~d}, J=7.8 \mathrm{~Hz}, 1 \mathrm{H}), 6.76(\mathrm{t}, J=7.05 \mathrm{~Hz}, 2 \mathrm{H})$, 6.94-7.00 (m, 1H), 7.03-7.08 (m, 1H), 7.22-7.27 (m, 1H), $7.33(\mathrm{t}, J=7.5 \mathrm{~Hz}, 2 \mathrm{H}), 7.52(\mathrm{~d}, J=$ $7.5 \mathrm{~Hz}, 2 \mathrm{H}), 8.15-8.18(\mathrm{~m}, 1 \mathrm{H}), 8.20$ (br s, $1 \mathrm{H}) ;{ }^{13} \mathrm{C} \mathrm{NMR}\left(75 \mathrm{MHz}, \mathrm{CDCl}_{3}\right) \delta_{\mathrm{C}}: 34.0,34.2,47.3$, 56.6, 60.1, 74.9, 109.3, 122.0,124.4, 126.1, 126.3, 127.2, 128.5,129.0, 130.1, 130.7, 130.9, 132.2, 137.7, 141.7, 142.0, 177.3, 193.5; ESI-MS: m/z. Calcd: 426.14; Found: 427.18(M+1).

\section{1'-Methyl-4'-(p-tolyl)dispiro[indoline-3,2'-pyrrolidine-3',3''-thiochromane]-2,4"'-dione}

4\{5,1,1\}: White solid. Yield: 80\%; mp (petroleum ether-ethyl acetate 1:4 v/v) $=220-221{ }^{\circ} \mathrm{C}$; IR $(\mathrm{KBr}) v_{\max }: 3173,2862,1693,1674,1621,1587,1472 \mathrm{~cm}^{-1} ;{ }^{1} \mathrm{H}$ NMR $\left(300 \mathrm{MHz}, \mathrm{CDCl}_{3}\right) \delta_{\mathrm{H}}$ : $2.10(\mathrm{~s}, 3 \mathrm{H}), 2.25(\mathrm{~s}, 3 \mathrm{H}), 2.63(\mathrm{~d}, J=14.4 \mathrm{~Hz}, 1 \mathrm{H}), 3.38(\mathrm{~d}, J=14.4 \mathrm{~Hz}, 1 \mathrm{H}), 3.41$ ( dd, $J=9.0$, $7.8 \mathrm{~Hz}, 1 \mathrm{H}), 3.97,(\mathrm{dd}, J=10.8,9.3 \mathrm{~Hz}, 1 \mathrm{H}), 5.16$ ( dd, $J=10.6,7.8 \mathrm{~Hz}, 1 \mathrm{H}), 6.54$ (td, $J=7.6$, $0.9 \mathrm{~Hz}, 1 \mathrm{H}), 6.66(\mathrm{~d}, J=7.5 \mathrm{~Hz}, 1 \mathrm{H}), 6.73-6.77(\mathrm{~m}, 2 \mathrm{H}), 6.93-7.08(\mathrm{~m}, 3 \mathrm{H}), 7.13(\mathrm{~d}, J=7.8 \mathrm{~Hz}$, 2H), 7.39 (d, $J=7.8 \mathrm{~Hz}, 2 \mathrm{H}), 8.04$ (br s, $1 \mathrm{H}), 8.14-8.17(\mathrm{~m}, 1 \mathrm{H}) ;{ }^{13} \mathrm{C} \mathrm{NMR}\left(75 \mathrm{MHz}, \mathrm{CDCl}_{3}\right)$ $\delta_{\mathrm{C}}: 21.0,34.0,34.2,47.0,56.6,60.0,74.9,109.3,122.0,124.3,126.2,126.3,127.2,128.9,129.1$, 130.0, 130.7, 130.8, 132.1, 134.5, 136.8, 141.7, 142.1, 177.5, 193.6; ESI-MS: m/z. Calcd: 
440.16; Found: $441.16(\mathrm{M}+1)$; Anal. Calcd for $\mathrm{C}_{27} \mathrm{H}_{24} \mathrm{~N}_{2} \mathrm{O}_{2} \mathrm{SC}$, 73.61; H, 5.49; N, 6.36; \%. Found C, 73.28; H, 5.53; N, $6.37 \%$.

4'-(2-Chlorophenyl)-1'-methyldispiro[indoline-3,2'-pyrrolidine-3',3'-thiochromane]-2,4"dione 4\{7,1,1\}: White solid. Yield: 73\%; $\mathrm{mp}$ (petroleum ether-ethyl acetate 1:4 v/v) $=239-240$ ${ }^{\circ} \mathrm{C}$; IR (KBr) v $v_{\max }: 3304,2926,1710,1658,1619,1587,1470 \mathrm{~cm}^{-1}$; ${ }^{1} \mathrm{H}$ NMR $\left(300 \mathrm{MHz}, \mathrm{CDCl}_{3}\right)$ $\delta_{\mathrm{H}}: 2.10(\mathrm{~s}, 3 \mathrm{H}), 2.70(\mathrm{~d}, J=14.1 \mathrm{~Hz}, 1 \mathrm{H}), 3.09$ (d, $\left.J=14.1 \mathrm{~Hz}, 1 \mathrm{H}\right), 3.52$ (t, $\left.J=8.5 \mathrm{~Hz}, 1 \mathrm{H}\right)$, $4.01(\mathrm{t}, J=9.4 \mathrm{~Hz}, 1 \mathrm{H}), 5.42(\mathrm{t}, J=9.0 \mathrm{~Hz}, 1 \mathrm{H}), 6.55(\mathrm{t}, J=7.6 \mathrm{~Hz}, 1 \mathrm{H}), 6.67(\mathrm{~d}, J=7.8 \mathrm{~Hz}$, $1 \mathrm{H}), 6.75-6.78(\mathrm{~m}, 2 \mathrm{H}), 6.98(\mathrm{td}, J=7.7,1.2 \mathrm{~Hz}, 1 \mathrm{H}), 7.05-7.12(\mathrm{~m}, 2 \mathrm{H}), 7.17-7.23(\mathrm{~m}, 1 \mathrm{H})$, $7.32-7.38(\mathrm{~m}, 2 \mathrm{H}), 7.75(\mathrm{br} \mathrm{s}, 1 \mathrm{H}), 8.09(\mathrm{~d}, J=8.1 \mathrm{~Hz}, 1 \mathrm{H}), 8.29-8.32(\mathrm{~m}, 1 \mathrm{H}) ;{ }^{13} \mathrm{C}$ NMR $(75$ $\left.\mathrm{MHz}, \mathrm{CDCl}_{3}\right) \delta_{\mathrm{C}}: 33.4,34.3,44.0,56.8,59.3,75.8,109.3,122.0,124.7,125.5,126.5,126.9$, 127.4, 128.3, 129.0, 129.4, 130.9, 131.0, 131.4, 132.1, 136.0, 136.1, 141.8, 142.0, 176.8, 192.7; ESI-MS: m/z. Calcd: 460.10; Found: 459.19 (M-1).

\section{4'-(3-Fluorophenyl)-1'-methyldispiro[indoline-3,2'-pyrrolidine-3',3"-thiochromane]-2,4"'-}

dione 4\{8,1,1\}: White solid. Yield: 84\%; $\mathrm{mp}$ (acetonitrile) $=204-205{ }^{\circ} \mathrm{C}$; IR $(\mathrm{KBr}) v_{\max }: 3204$, 3035, 2867, 2787, 1694, 1674, 1619, 1587, $1473 \mathrm{~cm}^{-1}$; ${ }^{1} \mathrm{H}$ NMR (300 MHz, $\left.\mathrm{CDCl}_{3}\right) \delta_{\mathrm{H}}: 2.01(\mathrm{~s}$, $3 \mathrm{H}), 2.65(\mathrm{~d}, J=14.1 \mathrm{~Hz}, 1 \mathrm{H}), 3.37(\mathrm{~d}, J=14.4 \mathrm{~Hz}, 1 \mathrm{H}), 3.44(\mathrm{dd}, J=9.0,7.8 \mathrm{~Hz}, 1 \mathrm{H}), 3.94$ (dd, $J=10.6,9.1 \mathrm{~Hz}, 1 \mathrm{H}), 5.18$ (dd, $J=10.5,7.8 \mathrm{~Hz}, 1 \mathrm{H}), 6.57$ (td, $J=7.5,1.0 \mathrm{~Hz}, 1 \mathrm{H}), 6.66(\mathrm{~d}$, $J=7.8 \mathrm{~Hz}, 1 \mathrm{H}), 6.73-6.78(\mathrm{~m}, 2 \mathrm{H}), 6.92-7.01(\mathrm{~m}, 2 \mathrm{H}), 7.04$ (t, $J=2.2 \mathrm{~Hz}, 1 \mathrm{H}), 7.08$ (dd, $J=$ 7.2, $1.2 \mathrm{~Hz}, 1 \mathrm{H}), 7.25-7.32(\mathrm{~m}, 3 \mathrm{H}), 7.92(\mathrm{br} \mathrm{s}, 1 \mathrm{H}), 8.16$ (dd, $J=7.8,1.5 \mathrm{~Hz}, 1 \mathrm{H}) ;{ }^{13} \mathrm{C} \mathrm{NMR}$ $\left(75 \mathrm{MHz}, \mathrm{CDCl}_{3}\right) \delta_{\mathrm{C}}: 33.8,34.1,47.0,56.6,60.0,74.9,109.4,114.2\left({ }^{2} J_{\mathrm{C}, \mathrm{F}}=21.2 \mathrm{~Hz}\right), 116.9\left({ }^{2} J_{\mathrm{C}}\right.$, $\mathrm{F}=21.4 \mathrm{~Hz}), 122.0,124.5,125.9,126.3,127.1,129.0,129.8,130.6,130.9,132.3,140.4\left({ }^{3} J_{\mathrm{C}, \mathrm{F}}=\right.$ $7.0 \mathrm{~Hz}), 141.8,141.9,162.9\left({ }^{1} J_{\mathrm{C}, \mathrm{F}}=244.4 \mathrm{~Hz}\right), 177.5,193.3$; ESI-MS: m/z. Calcd: 444.13; Found: $445.15\left(\mathrm{M}^{+}\right)$.

\section{4'-(3-Bromophenyl)-1'-methyldispiro[indoline-3,2'-pyrrolidine-3',3"'thiochromane]-2,4"-}

dione 4\{9,1,1\}: White solid. Yield: 82\%; $\mathrm{mp}$ (petroleum ether-ethyl acetate 1:4 v/v) = 200-201 ${ }^{\circ} \mathrm{C}$; IR (KBr) v $v_{\max }: 3246,2939,2861,2796,1688,1616,1587,1471 \mathrm{~cm}^{-1} ;{ }^{1} \mathrm{H}$ NMR (300 $\left.\mathrm{MHz}, \mathrm{CDCl}_{3}\right) \delta_{\mathrm{H}}: 2.10(\mathrm{~s}, 3 \mathrm{H}), 2.64(\mathrm{~d}, J=14.4 \mathrm{~Hz}, 1 \mathrm{H}), 3.35(\mathrm{~d}, J=14.1 \mathrm{~Hz}, 1 \mathrm{H}), 3.43(\mathrm{t}, J=$ $8.4 \mathrm{~Hz}, 1 \mathrm{H}), 3.93(\mathrm{t}, J=9.7 \mathrm{~Hz}, 1 \mathrm{H}), 5.14(\mathrm{dd}, J=10.5,7.8 \mathrm{~Hz}, 1 \mathrm{H}), 6.57(\mathrm{t}, J=7.5 \mathrm{~Hz}, 1 \mathrm{H})$, $6.67(\mathrm{~d}, J=7.5 \mathrm{~Hz}, 1 \mathrm{H}), 6.72-6.78(\mathrm{~m}, 2 \mathrm{H}), 6.96(\mathrm{t}, J=7.6 \mathrm{~Hz}, 1 \mathrm{H}), 7.01-7.10(\mathrm{~m}, 2 \mathrm{H}), 7.20(\mathrm{t}$, $J=7.8 \mathrm{~Hz}, 1 \mathrm{H}), 7.39$ (d, $J=8.1 \mathrm{~Hz}, 1 \mathrm{H}), 7.46(\mathrm{~d}, J=7.2 \mathrm{~Hz}, 1 \mathrm{H}), 7.68(\mathrm{~s}, 1 \mathrm{H}), 7.84(\mathrm{~s}, 1 \mathrm{H})$, 
$8.16(\mathrm{dd}, J=7.5,1.8 \mathrm{~Hz}, 1 \mathrm{H}) ;{ }^{13} \mathrm{C} \mathrm{NMR}\left(75 \mathrm{MHz}, \mathrm{CDCl}_{3}\right) \delta_{\mathrm{C}}: 34.0,34.1,46.9,56.6,59.9,75.0$, $109.5,122.0,122.7,124.5,125.9,126.3,127.1,128.9,129.1,130.0,130.4,130.6,130.9,132.3$, 133.0, 140.3, 141.7, 142.0, 177.5, 193.2; ESI-MS: m/z. Calcd: 504.05; Found: 503.00 (M-1); Anal. Calcd for $\mathrm{C}_{26} \mathrm{H}_{21} \mathrm{BrN}_{2} \mathrm{O}_{2} \mathrm{SC}, 61.79 ; \mathrm{H}, 4.19 ; \mathrm{N}, 5.54 ; \%$. Found C, 59.31; H, 4.30; N, 5.27 $\%$.

1'-Methyl-4'-(thiophen-2-yl)dispiro[indoline-3,2'-pyrrolidine-3',3'-thiochromane]-2,4"-

dione 4\{12,1,1\}: White solid. Yield: 75\%; $\mathrm{mp}$ (petroleum ether-ethyl acetate 1:4 v/v) = 219-220 ${ }^{\circ} \mathrm{C}$; IR (KBr) v $v_{\max }: 3276,2942,1717,1693,1651,1519,1468 \mathrm{~cm}^{-1}$; ${ }^{1} \mathrm{H}$ NMR (300 $\left.\mathrm{MHz}, \mathrm{CDCl}_{3}\right) \delta_{\mathrm{H}}: 2.10(\mathrm{~s}, 3 \mathrm{H}), 2.48(\mathrm{~d}, J=14.1 \mathrm{~Hz}, 1 \mathrm{H}), 3.48-3.57(\mathrm{~m}, 2 \mathrm{H}), 3.93$ (t, $J=9.3 \mathrm{~Hz}$, 1H), $5.41(\mathrm{dd}, J=10.5,7.8 \mathrm{~Hz}, 1 \mathrm{H}), 6.57$ (t, $J=7.6 \mathrm{~Hz}, 1 \mathrm{H}), 6.67$ (d, $J=6.0 \mathrm{~Hz}, 1 \mathrm{H}), 6.73(\mathrm{~d}, J$ $=7.5 \mathrm{~Hz}, 1 \mathrm{H}), 6.77(\mathrm{dd}, J=7.6,1.0 \mathrm{~Hz}, 1 \mathrm{H}), 6.95(\mathrm{dd}, J=7.8,1.2 \mathrm{~Hz}, 1 \mathrm{H}), 6.99-7.01(\mathrm{~m}, 1 \mathrm{H})$, $7.04(\mathrm{dd}, J=4.3,1.6 \mathrm{~Hz}, 1 \mathrm{H}), 7.08$ (dd, $J=7.2,1.5 \mathrm{~Hz}, 1 \mathrm{H}), 7.14$ (d, $J=3.3 \mathrm{~Hz}, 1 \mathrm{H}), 7.22$ (dd, $J$ $=5.1,0.9 \mathrm{~Hz}, 1 \mathrm{H}), 8.15(\mathrm{dd}, J=7.8,1.5 \mathrm{~Hz}, 1 \mathrm{H}) 8.31($ br s, $1 \mathrm{H}) ;{ }^{13} \mathrm{C} \mathrm{NMR}\left(75 \mathrm{MHz}, \mathrm{CDCl}_{3}\right) \delta_{\mathrm{C}}$ : 32.9, 34.0, 42.7, 58.1, 59.7, 74.9, 109.3, 122.0, 124.4, 124.7, 125.9, 126.3, 127.0, 127.1, 127.3, 129.0, 130.7, 130.8, 132.3, 140.6, 141.8, 142.1, 177.2, 193.2; ESI-MS: m/z. Calcd: 432.10; Found: 431.18(M-1); Anal. Calcd forC ${ }_{24} \mathrm{H}_{20} \mathrm{~N}_{2} \mathrm{O}_{2} \mathrm{~S}_{2} \mathrm{C}$, 66.64; H, 4.66; N, 6.48 \%. Found C, 64.70; H, 4.69; N, $6.28 \%$.

4'-(4-Chlorophenyl)-1',6"'-dimethyldispiro[indoline-3,2'-pyrrolidine-3',3'"-thiochromane]2,4"-dione 4\{13,1,1\}: White solid. Yield: 77\%; mp (petroleum ether-ethyl acetate 1:4 v/v) = $237-238^{\circ} \mathrm{C}$; IR (KBr) v $v_{\max }: 3119,2972,1689,1608,1651,1523,1473,1095 \mathrm{~cm}^{-1}$; ${ }^{1} \mathrm{H}$ NMR $\left(300 \mathrm{MHz}, \mathrm{CDCl}_{3}\right) \delta_{\mathrm{H}}: 2.08(\mathrm{~s}, 3 \mathrm{H}), 2.26(\mathrm{~s}, 3 \mathrm{H}), 2.57(\mathrm{~d}, J=14.4 \mathrm{~Hz}, 1 \mathrm{H}), 3.31(\mathrm{~d}, \mathrm{~J}=14.4 \mathrm{~Hz}$, $1 \mathrm{H}), 3.42(\mathrm{t}, \mathrm{J}=8.4 \mathrm{~Hz}, 1 \mathrm{H}), 3.91(\mathrm{t}, J=9.1 \mathrm{~Hz}, 1 \mathrm{H}), 5.13(\mathrm{dd}, J=10.6,7.6 \mathrm{~Hz}, 1 \mathrm{H}), 6.56-6.67$ $(\mathrm{m}, 3 \mathrm{H}), 6.75(\mathrm{~d}, J=7.5 \mathrm{~Hz}, 1 \mathrm{H}), 6.90(\mathrm{~d}, J=8.1 \mathrm{~Hz}, 1 \mathrm{H}), 6.94-7.00(\mathrm{~m}, 1 \mathrm{H}), 7.26-7.30$ (m, 2H), $7.44(\mathrm{~d}, J=8.1 \mathrm{~Hz}, 2 \mathrm{H}), 7.77($ br s, $1 \mathrm{H}), 7.96(\mathrm{~s}, 1 \mathrm{H}) ;{ }^{13} \mathrm{C} \mathrm{NMR}\left(75 \mathrm{MHz}, \mathrm{CDCl}_{3}\right) \delta_{\mathrm{C}}: 20.7$, $34.1,34.2$, 46.8, 56.7, 60.1, 74.9, 109.2, 122.0, 126.1, 126.3, 127.2, 128.6, 129.0, 130.5, 130.8, $131.5,133.0,133.5,134.2,136.4,136.8,141.6,177.1,193.6$;

1',6"'-Dimethyl-4'-(p-tolyl)dispiro[indoline-3,2'-pyrrolidine-3',3"'-thiochromane]-2,4' '-dione 4\{14,1,1\}: White solid. Yield: 70\%; mp (petroleum ether-ethyl acetate 1:4 v/v) $=235-236^{\circ} \mathrm{C}$; IR $(\mathrm{KBr}) v_{\max }: 3016,2931,1681,1604,1475,1396,1095 \mathrm{~cm}^{-1}$; ${ }^{1} \mathrm{H}$ NMR $\left(300 \mathrm{MHz}, \mathrm{CDCl}_{3}\right) \delta_{\mathrm{H}}$ : $2.09(\mathrm{~s}, 3 \mathrm{H}), 2.25(\mathrm{~s}, 3 \mathrm{H}), 2.32(\mathrm{~s}, 3 \mathrm{H}), 2.59(\mathrm{~d}, J=14.4 \mathrm{~Hz}, 1 \mathrm{H}), 3.33-3.43(\mathrm{~m}, 2 \mathrm{H}), 3.96(\mathrm{t}, J=$ 
$10.2 \mathrm{~Hz}, 1 \mathrm{H}), 5.13(\mathrm{dd}, J=11.1,8.4 \mathrm{~Hz}, 1 \mathrm{H}), 6.56-6.77(\mathrm{~m}, 3 \mathrm{H}), 6.77(\mathrm{~d}, J=7.2 \mathrm{~Hz}, 1 \mathrm{H}), 6.89$ $(\mathrm{d}, J=7.8 \mathrm{~Hz}, 1 \mathrm{H}), 6.96(\mathrm{t}, J=7.6 \mathrm{~Hz}, 1 \mathrm{H}), 7.13(\mathrm{~d}, J=7.8 \mathrm{~Hz}, 2 \mathrm{H}), 7.37$ (d, $J=7.5 \mathrm{~Hz}, 2 \mathrm{H})$, 7.55 (br s, $1 \mathrm{H}), 7.96(\mathrm{~s}, 1 \mathrm{H}) ;{ }^{13} \mathrm{C} \mathrm{NMR}\left(75 \mathrm{MHz}, \mathrm{CDCl}_{3}\right) \delta_{\mathrm{C}}: 20.7,21.0,34.2,47.1,56.6,60.3$, 74.8, 109.1, 121.9, 126.3, 127.3, 128.9, 129.1, 130.0, 130.6, 130.8, 133.4, 134.1, 134.6, 136.8, $138.8,141.6,177.0,193.9$.

\section{5-Chloro-4'-(4-chlorophenyl)-1'-methyldispiro[indoline-3,2'-pyrrolidine-3',3"}

thiochromane]-2,4"-dione 4\{2,2,1\}: White solid. Yield: $82 \%$; $\mathrm{mp}$ (petroleum ether-ethyl acetate $1: 4 \mathrm{v} / \mathrm{v})=223-224^{\circ} \mathrm{C}$; IR (KBr) $v_{\max }: 3314,2951,2847,1708,1686,1618,1588,1473 \mathrm{~cm}^{-1}$; ${ }^{1} \mathrm{H}$ NMR $\left(300 \mathrm{MHz}, \mathrm{CDCl}_{3}\right) \delta_{\mathrm{H}}: 2.10(\mathrm{~s}, 3 \mathrm{H}), 2.60(\mathrm{~d}, J=14.1 \mathrm{~Hz}, 1 \mathrm{H}), 3.28(\mathrm{~d}, J=14.4 \mathrm{~Hz}$, 1H), $3.42(\mathrm{t}, J=8.4 \mathrm{~Hz}, 1 \mathrm{H}), 3.89$ (t, $J=9.9 \mathrm{~Hz}, 1 \mathrm{H}), 5.16(\mathrm{dd}, J=10.5,7.8 \mathrm{~Hz}, 1 \mathrm{H}), 6.58(\mathrm{~d}, J$ $=8.1,1 \mathrm{H}), 6.71(\mathrm{~d}, J=2.1 \mathrm{~Hz}, 1 \mathrm{H}), 6.79-6.74(\mathrm{~m}, 1 \mathrm{H}), 6.93(\mathrm{dd}, J=8.1,2.1 \mathrm{~Hz}, 1 \mathrm{H}), 7.07-7.14$ (m, 2H), 7.30 (d, $J=8.4 \mathrm{~Hz}, 2 \mathrm{H}), 7.45$ (d, $J=7.8 \mathrm{~Hz}, 2 \mathrm{H}), 7.74(\mathrm{br} \mathrm{s}, 1 \mathrm{H}), 8.18-8.21$ (m, $1 \mathrm{H})$; ${ }^{13} \mathrm{C}$ NMR $\left(75 \mathrm{MHz}, \mathrm{CDCl}_{3}\right) \delta_{\mathrm{C}}: 33.7,34.2,46.3,56.8,60.1,74.8,110.2,124.8,126.2,127.6$, 127.7, 127.9, 128.7, 128.9, 130.6, 130.9, 131.5, 132.7, 133.2, 136.0, 140.0, 141.3, 176.9, 193.0; ESI-MS: m/z. Calcd: 494.06; Found: 493.14 (M-1); Anal. Calcd for $\mathrm{C}_{26} \mathrm{H}_{20} \mathrm{Cl}_{2} \mathrm{~N}_{2} \mathrm{O}_{2} \mathrm{~S}$ : C, 63.03; H, 4.07; N, $5.65 \%$. Found C, 62.80; H, 4.54; N, $5.45 \%$.

\section{5-Bromo-4'-(4-chlorophenyl)-1'-methyldispiro[indoline-3,2'-pyrrolidine-3',3'--}

thiochromane]-2,4"'-dione $\mathbf{4}\{\mathbf{2 , 3 , 1}\}$ : white solid. Yield: $80 \%$; mp (petroleum ether-ethyl acetate $1: 4 \mathrm{v} / \mathrm{v})=211-212^{\circ} \mathrm{C}$; IR (KBr) $v_{\max }: 3315,2952,1708,1656,1614,1494,1473 \mathrm{~cm}^{-1} ;{ }^{1} \mathrm{H}$ NMR $\left(300 \mathrm{MHz}, \mathrm{CDCl}_{3}\right) \delta_{\mathrm{H}}: 2.10(\mathrm{~s}, 3 \mathrm{H}), 2.60(\mathrm{~d}, J=14.4 \mathrm{~Hz}, 1 \mathrm{H}), 3.27$ (d, $\left.J=14.4 \mathrm{~Hz}, 1 \mathrm{H}\right), 3.42(\mathrm{t}$, $J=8.5 \mathrm{~Hz}, 1 \mathrm{H}), 3.89$ (t, $J=9.9 \mathrm{~Hz}, 1 \mathrm{H}), 5.16(\mathrm{dd}, J=10.5,7.8 \mathrm{~Hz}, 1 \mathrm{H}), 6.53(\mathrm{~d}, J=8.1,1 \mathrm{H})$, 6.75-6.78 (m, 1H), $6.84(\mathrm{~d}, J=1.5 \mathrm{~Hz}, 1 \mathrm{H}), 7.07$ (dd, $J=8.2,1.9 \mathrm{~Hz}, 1 \mathrm{H}), 7.10-7.13(\mathrm{~m}, 2 \mathrm{H})$, $7.30(\mathrm{~d}, J=8.4 \mathrm{~Hz}, 2 \mathrm{H}), 7.45(\mathrm{~d}, J=8.1 \mathrm{~Hz}, 2 \mathrm{H}), 7.76(\mathrm{br} \mathrm{s}, 1 \mathrm{H}), 8.18-8.21(\mathrm{~m}, 1 \mathrm{H}) ;{ }^{13} \mathrm{C} \mathrm{NMR}$ $\left(75 \mathrm{MHz}, \mathrm{CDCl}_{3}\right) \delta_{\mathrm{C}}: 33.6,34.2,46.3,56.8,60.1,74.8,110.7,114.9,124.9,126.2,128.2,128.7$, 130.5, 130.6, 131.0, 131.5, 131.7, 132.7, 136.0, 140.6, 141.2, 176.7, 193.0; ESI-MS: m/z. Calcd: 538.01; Found: $539.16\left(\mathrm{M}^{+}\right)$; Anal. Calcd for $\mathrm{C}_{26} \mathrm{H}_{20} \mathrm{BrClN}_{2} \mathrm{O}_{2} \mathrm{~S}$ : C, 57.84; H, 3.73; N, 5.19; \%. Found 57.81; H, 4.41; N, $4.76 \%$.

\section{7'-(4-Fluorophenyl)-7',7a'-dihydro-1'H,3' H-dispiro[indoline-3,5'-pyrrolo[1,2-c] thiazole-}

6',3"-thiochromane]-2,4"-dione 5\{1,1,2\}: White solid. Yield: 85\%; mp (petroleum ether-ethyl acetate $1: 4 \mathrm{v} / \mathrm{v})=236-237^{\circ} \mathrm{C}$; IR $(\mathrm{KBr}) v_{\max }: 3135,2934,2815,1694,1672,1620,1588,1473$ 
$\mathrm{cm}^{-1} ;{ }^{1} \mathrm{H}$ NMR $\left(300 \mathrm{MHz}, \mathrm{CDCl}_{3}\right) \delta_{\mathrm{H}}: 2.60(\mathrm{~d}, J=14.1 \mathrm{~Hz}, 1 \mathrm{H}), 2.82(\mathrm{t}, J=8.7 \mathrm{~Hz}, 1 \mathrm{H}), 2.95$ $(\mathrm{dd}, J=9.1,5.2 \mathrm{~Hz}, 1 \mathrm{H}), 3.39-3.53(\mathrm{~m}, 3 \mathrm{H}), 4.73-4.81(\mathrm{~m}, 1 \mathrm{H}), 4.87(\mathrm{~d}, J=9.9 \mathrm{~Hz}, 1 \mathrm{H})$, 6.58-6.64 (m, 1H), $6.66(\mathrm{~d}, J=7.5 \mathrm{~Hz}, 1 \mathrm{H}),(6.76, \mathrm{dd}, J=7.9,1.0 \mathrm{~Hz}, 1 \mathrm{H}),(6.86, \mathrm{~d}, J=7.8 \mathrm{~Hz}$, 1H), 6.94-7.00 (m, 2H), 7.01-7.10 (m, 3H), 7.44 (dd, $J=8.1,5.4 \mathrm{~Hz}, 2 \mathrm{H}), 7.88$ (br s, 1H), 8.06 $(\mathrm{dd}, J=8.1,1.5 \mathrm{~Hz}, 1 \mathrm{H}) ;{ }^{13} \mathrm{C} \mathrm{NMR}\left(75 \mathrm{MHz}, \mathrm{CDCl}_{3}\right) \delta_{\mathrm{C}}: 31.1,32.8,44.2,48.8,63.9,67.2,68.9$, $108.9,114.5\left({ }^{2} J_{\mathrm{C}, \mathrm{F}}=21.1 \mathrm{~Hz}\right), 120.2,123.5,123.9,125.2,125.7,128.2,129.6\left({ }^{2} J_{\mathrm{C}, \mathrm{F}}=20.4 \mathrm{~Hz}\right)$, $130.03\left({ }^{3} J_{\mathrm{C}, \mathrm{F}}=7.7 \mathrm{~Hz}\right), 131.5,131.6,140.7,141.5,161.0\left({ }^{1} J_{\mathrm{C}, \mathrm{F}}=244.3 \mathrm{~Hz}\right), 175.5,191.5$; ESIMS: m/z. Calcd: 488.10; Found: 487.14 (M-1); Anal. Calcd for $\mathrm{C}_{27} \mathrm{H}_{21} \mathrm{FN}_{2} \mathrm{O}_{2} \mathrm{~S}_{2}$ : C, 66.37; H, $4.33 ;$ N, 5.73; \%. Found C, 65.89; H, 4.49; N, $5.68 \%$.

7'-(4-Chlorophenyl)-7',7a'-dihydro-1' $H, 3$ ' $H$-dispiro[indoline-3,5'-pyrrolo[1,2-c]thiazole6',3'"-thiochromane]-2,4"'-dione: 5\{2,1,2\}: White solid. Yield: 80\%; mp (petroleum ether-ethyl acetate $1: 4 \mathrm{v} / \mathrm{v})=228-229^{\circ} \mathrm{C}$; IR $(\mathrm{KBr}) v_{\max }: 3198,2811,1694,1673,1620,1587,1473 \mathrm{~cm}^{-1}$; ${ }^{1} \mathrm{H}$ NMR $\left(300 \mathrm{MHz}, \mathrm{CDCl}_{3}\right) \delta_{\mathrm{H}}: 2.61(\mathrm{~d}, J=14.1 \mathrm{~Hz}, 1 \mathrm{H}), 2.82(\mathrm{t}, J=8.7 \mathrm{~Hz}, 1 \mathrm{H}), 2.94(\mathrm{dd}, J=$ 9.3, 5.1 Hz, 1H), 3.39-3.52 (m, 3H), 4.73-4.81 (m, 1H), $4.86(\mathrm{~d}, J=9.9 \mathrm{~Hz}, 1 \mathrm{H}), 6.61(\mathrm{t}, J=7.6$ $\mathrm{Hz}, 1 \mathrm{H}), 6.66(\mathrm{~d}, J=7.5 \mathrm{~Hz}, 1 \mathrm{H}), 6.77$ (d, J = 7.8 Hz, 1H), (6.86, d, J=7.5 Hz, 1H), 6.95-7.09 (m, 2H), 7.07 (t, $J=7.3 \mathrm{~Hz}, 1 \mathrm{H}), 7.32(\mathrm{~d}, J=8.1 \mathrm{~Hz}, 2 \mathrm{H}), 7.41(\mathrm{~d}, J=8.1 \mathrm{~Hz}, 2 \mathrm{H}), 7.93$ (br s, $1 \mathrm{H}), 8.06(\mathrm{~d}, J=7.8 \mathrm{~Hz}, 1 \mathrm{H}) ;{ }^{13} \mathrm{C} \mathrm{NMR}\left(75 \mathrm{MHz}, \mathrm{CDCl}_{3}\right) \delta_{\mathrm{C}}: 31.8,33.4,44.9,49.9,64.8,67.7$, $69.7,109.5,121.0,124.1,124.6,125.8,126.5,128.4,128.8,130.0,130.4,130.8,132.1,132.8$, 135.0, 141.3, 141.8, 176.3, 192.1; ESI-MS: m/z. Calcd: 504.07; Found: $505.14\left(\mathrm{M}^{+}\right)$; Anal. Calcd for $\mathrm{C}_{27} \mathrm{H}_{21} \mathrm{ClN}_{2} \mathrm{O}_{2} \mathrm{~S}_{2}$ : C, 64.21; H, 4.19; N, 5.55; \%. Found C, 64.17; H, 4.15; N, 5.61 \%.

\section{7'-(4-Bromophenyl)-7',7a'-dihydro-1' $H, 3$ ' $H$-dispiro[indoline-3,5'-pyrrolo[1,2-c] thiazole-}

6',3'"-thiochromane]-2,4"-dione 5\{3,1,2\}: White solid. Yield: 82\%; mp (petroleum ether-ethyl acetate $1: 4 \mathrm{v} / \mathrm{v})=225-226{ }^{\circ} \mathrm{C}$; IR $(\mathrm{KBr}) v_{\max }: 3180,2811,1694,1673,1620,1589,1472 \mathrm{~cm}^{-1}$; ${ }^{1} \mathrm{H}$ NMR $\left(300 \mathrm{MHz}, \mathrm{CDCl}_{3}\right) \delta_{\mathrm{H}}: 2.61(\mathrm{~d}, J=14.1 \mathrm{~Hz}, 1 \mathrm{H}), 2.81(\mathrm{t}, J=8.7 \mathrm{~Hz}, 1 \mathrm{H}), 2.94(\mathrm{dd}, J=$ $5.1,9.5 \mathrm{~Hz}, 1 \mathrm{H}), 3.38-3.51(\mathrm{~m}, 3 \mathrm{H}), 4.72-4.80(\mathrm{~m}, 1 \mathrm{H}), 4.84(\mathrm{~d}, J=9.9 \mathrm{~Hz}, 1 \mathrm{H}), 6.59(\mathrm{~d}, J=7.5$ $\mathrm{Hz}, 1 \mathrm{H}), 6.64(\mathrm{~d}, J=8.4 \mathrm{~Hz}, 1 \mathrm{H}), 6.77$ (d, $J=7.8 \mathrm{~Hz}, 1 \mathrm{H}), 6.85$ (d, $J=7.8 \mathrm{~Hz}, 1 \mathrm{H}), 6.95$ (d, $J=$ $7.5 \mathrm{~Hz}, 1 \mathrm{H}), 7.00(\mathrm{~d}, J=6.9 \mathrm{~Hz}, 1 \mathrm{H}), 7.05-7.10(\mathrm{~m}, 1 \mathrm{H}), 7.35$ (d, $J=8.1 \mathrm{~Hz}, 2 \mathrm{H}), 7.47$ (d, $J=$ $8.4 \mathrm{~Hz}, 2 \mathrm{H}), 7.65$ (br s, $1 \mathrm{H}), 8.06(\mathrm{~d}, J=7.8 \mathrm{~Hz}, 1 \mathrm{H}) ;{ }^{13} \mathrm{C} \mathrm{NMR}\left(75 \mathrm{MHz}, \mathrm{CDCl}_{3}\right) \delta_{\mathrm{C}}: 32.3,33.8$, 45.4, 50.0, 65.5, 68.1, 70.1, 109.7, 121.6, 122.2, 124.6, 125.1, 126.3, 127.4, 129.4, 130.4, 130.9, 131.5, 131.9, 132.6, 135.6, 140.9, 141.6, 176.7, 192.3; ESI-MS: m/z. Calcd: 548.02 Found: 
549.09 $\left(\mathrm{M}^{+}\right)$; Anal. Calcd for $\mathrm{C}_{27} \mathrm{H}_{21} \mathrm{BrN}_{2} \mathrm{O}_{2} \mathrm{~S}_{2}$ : C, 59.02; H, 3.85; N, 5.10; \%. Found C, 57.91; $\mathrm{H}, 4.06 ; \mathrm{N}, 5.40 \%$.

\section{7'-Phenyl-7',7a'-dihydro-1' H,3' H-dispiro[indoline-3,5'-pyrrolo[1,2-c]thiazole-6',3"'-}

thiochromane]-2,4"-dione 5\{4,1,2\}: White solid. Yield: 78\%; mp (petroleum ether-ethyl acetate $1: 4 \mathrm{v} / \mathrm{v})=231-232{ }^{\circ} \mathrm{C}$; IR $(\mathrm{KBr}) v_{\max }: 3196,2926,2818,1693,1676,1587,1473 \mathrm{~cm}^{-1}$; ${ }^{1} \mathrm{H}$ NMR $\left(300 \mathrm{MHz}, \mathrm{CDCl}_{3}\right) \delta_{\mathrm{H}}: 2.62(\mathrm{~d}, J=14.1 \mathrm{~Hz}, 1 \mathrm{H}), 2.83(\mathrm{t}, J=8.4 \mathrm{~Hz}, 1 \mathrm{H}), 2.95-2.99$ (m, 1H), 3.40-3.56 (m, 3H), 4.83-4.91 (m, 2H), 6.59-6.65 (m, 2H), 6.77 (d, $J=7.8 \mathrm{~Hz}, 1 \mathrm{H})$, $6.88(\mathrm{~d}, J=7.5 \mathrm{~Hz}, 1 \mathrm{H}), 6.97-6.99$ (m, 2H), 7.04-7.09 (m, 1H), 7.29-7.39 (m, 3H), 7.46 (d, $J=$ $7.2 \mathrm{~Hz}, 2 \mathrm{H}), 7.74(\mathrm{br} \mathrm{s}, 1 \mathrm{H}), 8.06(\mathrm{~d}, J=7.8 \mathrm{~Hz}, 1 \mathrm{H}) ;{ }^{13} \mathrm{C} \mathrm{NMR}\left(75 \mathrm{MHz}, \mathrm{CDCl}_{3}\right) \delta_{\mathrm{C}}: 30.9,32.8$, 44.5, 49.7, 64.2, 66.8, 68.8, 109.2, 120.5, 124.3, 125.9, 127.0, 128.3, 128.8, 129.5, 130.0, 132.4, 135.9, 140.5, 142.0, 175.4, 191.6; ESI-MS: m/z. Calcd: 470.11; Found: 469.16 (M-1); Anal. Calcd for $\mathrm{C}_{27} \mathrm{H}_{22} \mathrm{~N}_{2} \mathrm{O}_{2} \mathrm{~S}_{2}$ : C, 68.91; H, 4.71; N, 5.95; \%. Found C, 68.93; H, 4.84; N, $5.72 \%$.

\section{7'-(p-Tolyl)-7',7a'-dihydro-1'H,3' H-dispiro[indoline-3,5'-pyrrolo[1,2-c]thiazole-6',3'"-}

thiochromane]-2,4"-dione 5\{5,1,2\}: White solid. Yield: 81\%; mp (petroleum ether-ethyl acetate $1: 4 \mathrm{v} / \mathrm{v})=243-244{ }^{\circ} \mathrm{C}$; IR (KBr) $v_{\max }: 3186,2936,1687,1679,1618,1586,1472 \mathrm{~cm}^{-1} ;{ }^{1} \mathrm{H}$ NMR $\left(300 \mathrm{MHz}, \mathrm{CDCl}_{3}\right) \delta_{\mathrm{H}}: 2.37(\mathrm{~s}, 3 \mathrm{H}), 2.63(\mathrm{~d}, J=14.4 \mathrm{~Hz}, 1 \mathrm{H}), 2.82(\mathrm{t}, J=8.4 \mathrm{~Hz}, 1 \mathrm{H}), 2.96(\mathrm{dd}$, $J=9.0,4.2 \mathrm{~Hz}, 1 \mathrm{H}), 3.39-3.55(\mathrm{~m}, 3 \mathrm{H}), 4.80-4.87(\mathrm{~m}, 2 \mathrm{H}), 6.59-6.64(\mathrm{~m}, 2 \mathrm{H}), 6.76-6.78(\mathrm{~m}$, $1 \mathrm{H}), 6.88(\mathrm{~d}, J=7.5 \mathrm{~Hz}, 1 \mathrm{H}), 6.93-7.00(\mathrm{~m}, 2 \mathrm{H}), 7.05$ (td, $J=7.4,1.6 \mathrm{~Hz}, 1 \mathrm{H}), 7.14$ (d, $J=7.8$ $\mathrm{Hz}, 2 \mathrm{H}), 7.34$ (d, $J=7.8 \mathrm{~Hz}, 2 \mathrm{H}), 7.70($ br s, $1 \mathrm{H}), 8.05$ (dd, $J=7.9,1.3 \mathrm{~Hz}, 1 \mathrm{H}) ;{ }^{13} \mathrm{C}$ NMR $(75$ $\left.\mathrm{MHz}, \mathrm{CDCl}_{3}\right) \delta_{\mathrm{C}}: 20.2,31.3,32.9,44.5,49.4,64.3,67.0,69.1,109.0,120.4,123.6,124.3,125.4$, 126.0, 128.3, 128.5, 128.7, 129.7, 129.9, 131.5, 132.7, 136.1, 140.9, 141.6, 175.8, 191.8; ESIMS: m/z. Calcd: 484.13; Found: 483.22 (M-1).

7'-(4-Methoxyphenyl)-7',7a'-dihydro-1' $H, 3^{\prime} H$-dispiro[indoline-3,5'-pyrrolo[1,2-c]thiazole$\mathbf{6}^{\prime}, 3^{3}$ '-thiochromane]-2,4"-dione 5\{6,1,2\}: White solid. Yield: 74\%; mp (petroleum ether-ethyl acetate $1: 4 \mathrm{v} / \mathrm{v})=227-228^{\circ} \mathrm{C}$; IR $(\mathrm{KBr}) v_{\max }: 3200,2925,2817,1694,1617,1587,1487 \mathrm{~cm}^{-1}$; ${ }^{1} \mathrm{H} \mathrm{NMR}\left(300 \mathrm{MHz}, \mathrm{CDCl}_{3}\right) \delta_{\mathrm{H}}: 2.63(\mathrm{~d}, J=14.4 \mathrm{~Hz}, 1 \mathrm{H}), 2.82(\mathrm{t}, J=8.4 \mathrm{~Hz}, 1 \mathrm{H}), 2.96(\mathrm{dd}, J=$ 9.1, 4.6 Hz, 1H), 3.39-3.54 (m, 3H), 3.79 (s, 3H), 4.73-4.85 (m, 2H), 6.58-6.65 (m, 2H), 6.76-6.78 (m,1H), 6.87 (d, $J=7.8 \mathrm{~Hz}, 3 \mathrm{H}), 6.93-7.00(\mathrm{~m}, 2 \mathrm{H}), 7.06$ (td, $J=7.5,1.5 \mathrm{~Hz}, 1 \mathrm{H})$, 7.37 (d, $J=8.4 \mathrm{~Hz}, 2 \mathrm{H}), 7.76($ br s, $1 \mathrm{H}), 8.05$ (dd, $J=7.9,1.3 \mathrm{~Hz}, 1 \mathrm{H}) ;{ }^{13} \mathrm{C}$ NMR $(75 \mathrm{MHz}$, $\left.\mathrm{CDCl}_{3}\right) \delta_{\mathrm{C}}: 32.3,33.7,45.5,49.9,55.2,65.5,68.2,70.0,109.4,114.1,122.1,124.4,125.3,126.2$, 
127.5, 128.3, 129.3, 130.5, 130.7, 130.8, 132.4, 140.8, 141.7, 159.0, 176.6, 192.5; ESI-MS: m/z. Calcd:500.12; Found: $501.15\left(\mathrm{M}^{+}\right)$.

\section{7'-(2-Chlorophenyl)-7',7a'-dihydro-1'H,3'H-dispiro[indoline-3,5'-pyrrolo[1,2-c] thiazole-} 6',3"'-thiochromane]-2,4"-dione 5\{7,1,2\}: White solid. Yield: 78\%; mp (petroleum ether-ethyl acetate $1: 4 \mathrm{v} / \mathrm{v})=209-210{ }^{\circ} \mathrm{C}$; IR $(\mathrm{KBr}) v_{\max }: 3375,3166,2924,2819,1705,1693,1620,1585$, $1473 \mathrm{~cm}^{-1}$; ${ }^{1} \mathrm{H}$ NMR $\left(300 \mathrm{MHz}, \mathrm{CDCl}_{3}\right) \delta_{\mathrm{H}}: 2.67(\mathrm{~d}, J=14.1 \mathrm{~Hz}, 1 \mathrm{H}), 2.93(\mathrm{~d}, J=7.5 \mathrm{~Hz}, 2 \mathrm{H})$, $3.23(\mathrm{~d}, J=14.1 \mathrm{~Hz}, 1 \mathrm{H}), 3.35$ (d, $J=5.1 \mathrm{~Hz}, 1 \mathrm{H}), 3.34$ (d, $J=5.1 \mathrm{~Hz}, 1 \mathrm{H}), 4.81$ (dd, $J=15.3$, $7.8 \mathrm{~Hz}, 1 \mathrm{H}), 5.12(\mathrm{~d}, J=8.7 \mathrm{~Hz}, 1 \mathrm{H}), 6.56(\mathrm{t}, J=7.5 \mathrm{~Hz}, 1 \mathrm{H}), 6.69(\mathrm{~d}, J=7.5 \mathrm{~Hz}, 1 \mathrm{H})$, 6.76-6.80 (m, 1H), $6.83(\mathrm{~d}, J=7.5 \mathrm{~Hz}, 1 \mathrm{H}), 6.99(\mathrm{td}, J=7.7,1.1 \mathrm{~Hz}, 1 \mathrm{H}), 7.04-7.12(\mathrm{~m}, 2 \mathrm{H})$, 7.20-7.25 (m, 1H), 7.34-7.40 (m, 2H), 7.92 (br s, 1H), 8.05 (d, J=7.8 Hz, 1H), 8.25-8.29 (m, $1 \mathrm{H}) ;{ }^{13} \mathrm{C}$ NMR $\left(75 \mathrm{MHz}, \mathrm{CDCl}_{3}\right) \delta_{\mathrm{C}}: 32.4,33.5,44.6,47.1,64.9,69.2,70.8,109.7,122.0,124.6$, 124.9, 126.5, 127.1, 127.5, 128.6, 129.3, 129.6, 130.9, 132.3, 135.3, 136.1, 141.2, 141.8, 176.3, 191.5; ESI-MS: m/z. Calcd: 504.07; Found: 503.16 (M-1).

7'-(3-Fluorophenyl)-7',7a'-dihydro-1' $H, 3$ ' $H$-dispiro[indoline-3,5'-pyrrolo[1,2-c]thiazole6',3"-thiochromane]-2,4"-dione 5\{8,1,2\}: White solid. Yield: 83\%; mp (petroleum ether-ethyl acetate $1: 4 \mathrm{v} / \mathrm{v})=214-215^{\circ} \mathrm{C}$; IR $(\mathrm{KBr}) v_{\max }: 3214,2924,2852,1698,1672,1615,1515,1470$ $\mathrm{cm}^{-1} ;{ }^{1} \mathrm{H}$ NMR $\left(300 \mathrm{MHz}, \mathrm{CDCl}_{3}\right) \delta_{\mathrm{H}}: 2.65(\mathrm{~d}, J=14.1 \mathrm{~Hz}, 1 \mathrm{H}), 2.82(\mathrm{t}, J=8.7 \mathrm{~Hz}, 1 \mathrm{H}), 2.97$ (dd, $J=9.1,5.2 \mathrm{~Hz}, 1 \mathrm{H}), 3.40$ (d, $J=5.1 \mathrm{~Hz}, 1 \mathrm{H}), 3.47-3.56(\mathrm{~m}, 2 \mathrm{H}), 4.75-4.83(\mathrm{~m}, 1 \mathrm{H}), 4.89$ $(\mathrm{d}, J=9.9 \mathrm{~Hz}, 1 \mathrm{H}), 6.61(\mathrm{t}, J=7.6 \mathrm{~Hz}, 1 \mathrm{H}), 6.66(\mathrm{~d}, J=7.8 \mathrm{~Hz}, 1 \mathrm{H}), 6.78(\mathrm{~d}, J=8.1 \mathrm{~Hz}, 1 \mathrm{H})$, $6.86(\mathrm{~d}, J=7.5 \mathrm{~Hz}, 1 \mathrm{H}), 6.95-7.01(\mathrm{~m}, 3 \mathrm{H}), 7.07(\mathrm{td}, J=7.4,1.6 \mathrm{~Hz}, 1 \mathrm{H}), 7.22(\mathrm{~d}, J=7.8 \mathrm{~Hz}$, 2H), $7.32(\mathrm{t}, J=6.9 \mathrm{~Hz}, 1 \mathrm{H}), 7.83(\mathrm{br} \mathrm{s}, 1 \mathrm{H}), 8.07(\mathrm{dd}, J=7.9,1.3 \mathrm{~Hz}, 1 \mathrm{H}) ;{ }^{13} \mathrm{C}$ NMR $(75 \mathrm{MHz}$, $\left.\mathrm{CDCl}_{3}\right) \delta_{\mathrm{C}}: 32.2,33.6,45.4,50.2,65.6,68.0,70.1,109.7,114.5\left({ }^{2} J_{\mathrm{C}, \mathrm{F}}=20.9 \mathrm{~Hz}\right), 116.6\left({ }^{2} J_{\mathrm{C}, \mathrm{F}}=\right.$ $21.3 \mathrm{~Hz}), 122.1,124.5,125.1,125.5,126.2,127.4,129.4,130.1\left({ }^{3} J_{\mathrm{C}, \mathrm{F}}=8.2 \mathrm{~Hz}\right), 130.4,130.8$, 132.5, $139.2\left({ }^{3} J_{\mathrm{C}, \mathrm{F}}=7.1 \mathrm{~Hz}\right), 141.0,141.5,162.7\left({ }^{1} J_{\mathrm{C}, \mathrm{F}}=245.2 \mathrm{~Hz}\right), 177.0,192.2 ;$ ESI-MS: $\mathrm{m} / \mathrm{z}$. Calcd: 488.10; Found: 487.14 (M-1).

\section{7'-(3-Bromophenyl)-7',7a'-dihydro-1' $H, 3$ ' $H$-dispiro[indoline-3,5'-pyrrolo[1,2-c] thiazole-} 6',3"-thiochromane]-2,4"-dione 5\{9,1,2\}: White solid. Yield: 82\%; mp (petroleum ether-ethyl acetate $1: 4 \mathrm{v} / \mathrm{v})=206-207^{\circ} \mathrm{C}$; IR (KBr) $v_{\max }: 3154,2920,2851,1700,1674,1618,1588,1492$ $\mathrm{cm}^{-1} ;{ }^{1} \mathrm{H}$ NMR $\left(300 \mathrm{MHz}, \mathrm{CDCl}_{3}\right) \delta_{\mathrm{H}}: 2.64(\mathrm{~d}, J=14.1 \mathrm{~Hz}, 1 \mathrm{H}), 2.82(\mathrm{t}, J=8.7 \mathrm{~Hz}, 1 \mathrm{H}), 2.96$ (dd, $J=9.1,5.1 \mathrm{~Hz}, 1 \mathrm{H}), 3.38-3.54(\mathrm{~m}, 3 \mathrm{H}), 4.73-4.81(\mathrm{~m}, 1 \mathrm{H}), 4.85(\mathrm{~d}, J=9.9 \mathrm{~Hz}, 1 \mathrm{H}), 6.61$ 
$(\mathrm{t}, J=7.6 \mathrm{~Hz}, 1 \mathrm{H}), 6.66(\mathrm{~d}, J=8.1 \mathrm{~Hz}, 1 \mathrm{H}), 6.78(\mathrm{~d}, J=8.1 \mathrm{~Hz}, 1 \mathrm{H}), 6.85(\mathrm{~d}, J=7.8 \mathrm{~Hz}, 1 \mathrm{H})$, $6.96(\mathrm{~d}, J=6.9 \mathrm{~Hz}, 1 \mathrm{H}), 7.00(\mathrm{~d}, J=6.3 \mathrm{~Hz}, 1 \mathrm{H}), 7.08(\mathrm{t}, J=6.6 \mathrm{~Hz}, 1 \mathrm{H}), 7.22$ (t, $J=7.9 \mathrm{~Hz}$, 1H), 7.42 (d, $J=7.5 \mathrm{~Hz}, 2 \mathrm{H}), 7.61(\mathrm{~s}, 1 \mathrm{H}), 7.78$ (br s, $1 \mathrm{H}), 8.07$ (d, $J=7.5 \mathrm{~Hz}, 1 \mathrm{H}) ;{ }^{13} \mathrm{C}$ NMR $\left(75 \mathrm{MHz}, \mathrm{CDCl}_{3}\right) \delta_{\mathrm{C}}: 33.3,33.8,45.4,50.2,65.6,68.1,70.2,109.8,122.2,123.0,124.6,125.1$, $126.3,127.4,128.5,129.5,130.3,130.4,130.8,130.9$, 132.6, 139.2, 141.0, 141.6, 177.0, 192.2; ESI-MS: m/z. Calcd: 548.02; Found: $548.96\left(\mathrm{M}^{+}\right)$.

7'-(2,4-Dichlorophenyl)-7',7a'-dihydro-1' $H, 3$ ' $H$-dispiro[indoline-3,5'-pyrrolo[1,2-c] thiazole$6^{\prime}, 3^{\prime \prime}$-thiochromane]-2,4"-dione 5\{10,1,2\}: White solid. Yield: 78\%; mp (petroleum etherethyl acetate $1: 4 \mathrm{v} / \mathrm{v})=230-231{ }^{\circ} \mathrm{C}$; IR $(\mathrm{KBr}) v_{\max }: 3184,2925,2815,1697,1620,1588 \mathrm{~cm}^{-1} ;{ }^{1} \mathrm{H}$ NMR $\left(300 \mathrm{MHz}, \mathrm{CDCl}_{3}\right) \delta_{\mathrm{H}}: 2.65(\mathrm{~d}, J=14.1 \mathrm{~Hz}, 1 \mathrm{H}), 2.90-2.93(\mathrm{~m}, 2 \mathrm{H}), 3.20(\mathrm{~d}, J=14.1 \mathrm{~Hz}$, $1 \mathrm{H}), 3.33(\mathrm{~d}, J=5.1 \mathrm{~Hz}, 1 \mathrm{H}), 3.38(\mathrm{~d}, J=5.4 \mathrm{~Hz}, 1 \mathrm{H}), 4.70-4.75(\mathrm{~m}, 1 \mathrm{H}), 5.09$ (d, $J=8.4 \mathrm{~Hz}$, $1 \mathrm{H}), 6.56(\mathrm{t}, J=7.6 \mathrm{~Hz}, 1 \mathrm{H}), 6.68(\mathrm{~d}, J=7.8 \mathrm{~Hz}, 1 \mathrm{H}), 6.76-6.82(\mathrm{~m}, 2 \mathrm{H}), 6.99(\mathrm{t}, J=7.2 \mathrm{~Hz}$, 1H), 7.06-7.13 (m, 2H), 7.35-7.39 (m, 2H), 7.67 (br s, 1H), 8.01 (d, J=9.3 Hz, 1H), 8.24-8.27 $(\mathrm{m}, 1 \mathrm{H}) ;{ }^{13} \mathrm{C} \mathrm{NMR}\left(75 \mathrm{MHz}, \mathrm{CDCl}_{3}\right) \delta_{\mathrm{C}}: 32.3,33.5,44.6,46.6,64.8,69.4,70.8,109.7,122.1$, $124.4,125.0,126.5,127.5,129.4,130.8,130.9,131.9,132.4,133.8,134.1,136.7,141.1,141.7$, 176.1, 191.4; ESI-MS: m/z. Calcd: 538.03; Found: 537.08 (M-1).

\section{7'-(Naphthalen-1-yl)-7',7a'-dihydro-1' $H, 3$ ' $H$-dispiro[indoline-3,5'-pyrrolo[1,2-c]thiazole-}

6',3"-thiochromane]-2,4"'-dione 5\{11,1,2\}: White solid. Yield: 72\%; mp (petroleum etherethyl acetate $1: 4 \mathrm{v} / \mathrm{v})=218-219{ }^{\circ} \mathrm{C}$; IR $(\mathrm{KBr}) v_{\max }: 3196,2821,1696,1674,1584,1472 \mathrm{~cm}^{-1} ;{ }^{1} \mathrm{H}$ NMR $\left(300 \mathrm{MHz}, \mathrm{CDCl}_{3}\right) \delta_{\mathrm{H}}: 2.34(\mathrm{~d}, J=14.1 \mathrm{~Hz}, 1 \mathrm{H}), 2.86-2.95(\mathrm{~m}, 2 \mathrm{H}), 3.42-3.50(\mathrm{~m}, 3 \mathrm{H})$, $5.06(\mathrm{dd}, J=14.8,8.5 \mathrm{~Hz}, 1 \mathrm{H}), 5.56(\mathrm{~d}, J=9.3 \mathrm{~Hz}, 1 \mathrm{H}), 6.59(\mathrm{t}, J=7.5 \mathrm{~Hz}, 1 \mathrm{H}), 6.70(\mathrm{~d}, J=$ $7.5 \mathrm{~Hz}, 2 \mathrm{H}), 6.90(\mathrm{~d}, J=7.8 \mathrm{~Hz}, 1 \mathrm{H}), 6.97-7.06(\mathrm{~m}, 3 \mathrm{H}), 7.42-7.53(\mathrm{~m}, 2 \mathrm{H}), 7.56$ (t, $J=7.6 \mathrm{~Hz}$, 1H), 7.79 (br s, 1H), 7.85 (dd, $J=12.4,4.5 \mathrm{~Hz}, 2 \mathrm{H}), 8.08$ (d, J = 8.1 Hz, 1H), 8.14 (d, $J=7.5 \mathrm{~Hz}$, $1 \mathrm{H}), 8.30(\mathrm{dd}, J=5.7,3.9 \mathrm{~Hz}, 1 \mathrm{H}) ;{ }^{13} \mathrm{C} \mathrm{NMR}\left(75 \mathrm{MHz}, \mathrm{CDCl}_{3}+\mathrm{DMSO}^{6}{ }^{6}\right) \delta_{\mathrm{C}}: 31.3,32.5,43.8$, 44.3, 64.0, 68.4, 69.5, 109.0, 120.2, 122.7, 123.7, 123.8, 124.6, 125.4, 125.7, 125.8, 126.5, 126.9, 127.9, 128.2, 129.7, 129.8, 131.5, 132.2, 132.5, 132.8, 141.1, 141.7, 175.2, 191.9; ESI-MS: m/z. Calcd: 520.13; Found: 519.21(M-1); Anal. Calcd for $\mathrm{C}_{31} \mathrm{H}_{24} \mathrm{~N}_{2} \mathrm{O}_{2} \mathrm{~S}_{2}$ : C, 71.51; H, 4.65; N, 5.38; \%. Found C, 69.99; H, 4.74; N, $5.31 \%$.

\section{7'-(Thiophen-2-yl)-7',7a'-dihydro-1' $H, 3$ ' $H$-dispiro[indoline-3,5'-pyrrolo[1,2-c]thiazole-}

6',3"-thiochromane]-2,4"'-dione 5\{12,1,2\}: White solid. Yield: 74\%; mp (petroleum ether- 
ethyl acetate $1: 4 \mathrm{v} / \mathrm{v})=207-208{ }^{\circ} \mathrm{C}$; IR (KBr) $v_{\max }: 3204,1702,1659,1618,1583,1474 \mathrm{~cm}^{-1} ;{ }^{1} \mathrm{H}$ NMR $\left(300 \mathrm{MHz}, \mathrm{CDCl}_{3}\right) \delta_{\mathrm{H}}: 2.87-2.91(\mathrm{~m}, 2 \mathrm{H}), 3.06(\mathrm{dd}, J=9.3,5.4 \mathrm{~Hz}, 1 \mathrm{H}), 3.42(\mathrm{~d}, J=5.7$ $\mathrm{Hz}, 1 \mathrm{H}), 3.53(\mathrm{~d}, J=5.4 \mathrm{~Hz}, 1 \mathrm{H}), 3.66(\mathrm{~d}, J=14.1 \mathrm{~Hz}, 1 \mathrm{H}), 4.73-4.77(\mathrm{~m}, 1 \mathrm{H}), 5.10(\mathrm{~d}, J=9.9$ $\mathrm{Hz}, 1 \mathrm{H}), 6.64(\mathrm{t}, J=7.8 \mathrm{~Hz}, 2 \mathrm{H}), 6.81(\mathrm{~d}, J=7.8 \mathrm{~Hz}, 1 \mathrm{H}), 6.90-7.10(\mathrm{~m}, 6 \mathrm{H}), 7.23-7.26(\mathrm{~m}$, 1H), 7.85 (br s, 1H), $7.96(\mathrm{~d}, J=8.1 \mathrm{~Hz}, 1 \mathrm{H}) ;{ }^{13} \mathrm{C} \mathrm{NMR}\left(75 \mathrm{MHz}, \mathrm{CDCl}_{3}\right) \delta_{\mathrm{C}}: 32.4,32.7,46.3$, 46.4, 65.1, 69.4, 70.4, 109.6, 122.1, 124.4, 124.9, 125.0, 126.1, 127.1, 127.2, 127.5, 129.4, 130.3, 130.7, 132.6, 138.9, 141.1, 141.5, 177.2, 192.0; ESI-MS: m/z. Calcd: 476.07; Found: 475.11 (M1); Anal. Calcd forC ${ }_{25} \mathrm{H}_{20} \mathrm{~N}_{2} \mathrm{O}_{2} \mathrm{~S}_{3}$ : C, 63.00; H, 4.23; N, 5.88; \%. Found C, 62.24; H, 4.20; N, $5.82 \%$.

7'-(4-Chlorophenyl)-6'"-methyl-7',7a'-dihydro-1' H,3' $H$-dispiro[indoline-3,5'-pyrrolo[1,2c]thiazole-6',3"-thiochromane]-2,4"-dione 5\{13,1,2\}: White solid. Yield: 76\%; mp (petroleum ether-ethyl acetate $1: 4 \mathrm{v} / \mathrm{v})=232-233{ }^{\circ} \mathrm{C}$; IR (KBr) $v_{\max }: 3166,2916,2731,1681,1605,1396$, $1087 \mathrm{~cm}^{-1} ;{ }^{1} \mathrm{H}$ NMR $\left(300 \mathrm{MHz}, \mathrm{CDCl}_{3}\right) \delta_{\mathrm{H}}: 2.23(\mathrm{~s}, 3 \mathrm{H}), 2.58(\mathrm{~d}, J=14.1 \mathrm{~Hz}, 1 \mathrm{H}), 2.81(\mathrm{t}, J=$ $8.7 \mathrm{~Hz}, 1 \mathrm{H}), 2.94(\mathrm{dd}, J=9.0,5.1 \mathrm{~Hz}, 1 \mathrm{H}), 3.39-3.50(\mathrm{~m}, 3 \mathrm{H}), 4.75-4.80(\mathrm{~m}, 1 \mathrm{H}), 4.84(\mathrm{~d}, J=$ $9.9 \mathrm{~Hz}, 1 \mathrm{H}), 6.59-6.69(\mathrm{~m}, 3 \mathrm{H}), 6.85-6.91(\mathrm{~m}, 2 \mathrm{H}), 6.98(\mathrm{t}, J=7.6 \mathrm{~Hz}, 1 \mathrm{H}), 7.31(\mathrm{~d}, J=8.7$ $\mathrm{Hz}, 2 \mathrm{H}), 7.39(\mathrm{~d}, J=8.1 \mathrm{~Hz}, 2 \mathrm{H}), 7.62($ br s, $1 \mathrm{H}), 7.86(\mathrm{~s}, 1 \mathrm{H}) ;{ }^{13} \mathrm{C} \mathrm{NMR}\left(75 \mathrm{MHz}, \mathrm{CDCl}_{3}\right) \delta_{\mathrm{C}}$ : $19.3,30.5,32.5,43.7,48.5,63.7,66.5,68.4,108.4,119.6,123.6,124.8,125.2,127.3,127.8$, $128.7,129.2,129.7,131.5,132.3,132.6,134.1,136.8,141.2,175.0,191.1$.

6"'-Chloro-7'-(4-chlorophenyl)-7',7a'-dihydro-1'H,3' H-dispiro[indoline-3,5'-pyrrolo[1,2c]thiazole-6',3"-thiochromane]-2,4"-dione 5\{15,1,2\}: Light yellow solid. Yield: 84\%; mp (petroleum ether-ethyl acetate 1:4 v/v) $=233-234{ }^{\circ} \mathrm{C}$; IR $(\mathrm{KBr}) v_{\max }: 3178,2922,2835,1697$, 1676, 1413, $1097 \mathrm{~cm}^{-1} ;{ }^{1} \mathrm{H}$ NMR $\left(300 \mathrm{MHz}, \mathrm{CDCl}_{3}\right) \delta_{\mathrm{H}}: 2.55(\mathrm{~d}, J=14.4 \mathrm{~Hz}, 1 \mathrm{H}), 2.81(\mathrm{t}, J=$ $8.5 \mathrm{~Hz}, 1 \mathrm{H}), 2.91-2.96(\mathrm{~m}, 1 \mathrm{H}), 3.38-3.53(\mathrm{~m}, 3 \mathrm{H}), 4.72-4.83(\mathrm{~m}, 2 \mathrm{H}), 6.64-6.74(\mathrm{~m}, 3 \mathrm{H}), 6.84$ (d, $J=7.5 \mathrm{~Hz}, 1 \mathrm{H}), 6.99-7.05(\mathrm{~m}, 2 \mathrm{H}), 7.32$ (d, $J=8.4 \mathrm{~Hz}, 2 \mathrm{H}), 7.39(\mathrm{~d}, J=8.1 \mathrm{~Hz}, 2 \mathrm{H}), 7.61$ (br s, $1 \mathrm{H}), 8.01(\mathrm{~d}, J=2.4 \mathrm{~Hz}, 1 \mathrm{H}) ;{ }^{13} \mathrm{C} \mathrm{NMR}\left(75 \mathrm{MHz}, \mathrm{CDCl}_{3}\right) \delta_{\mathrm{C}}: 30.8,32.5,39.7,44.0,48.8$, $63.6,66.9$, 68.6, 109.0, 120.1, 123.5, 125.4, 126.7, 127.6, 128.3, 128.7, 129.0, 130.0,130.3, $131.2,132.0,134.0,139.0,141.5,175.2,190.3$.

6"'-Chloro-7'-(p-tolyl)-7',7a'-dihydro-1' $H, 3$ ' $H$-dispiro[indoline-3,5'-pyrrolo[1,2-c]thiazole6',3"'-thiochromane]-2,4"'-dione 5\{16,1,2\}: White solid. Yield: 82\%; mp (petroleum ether-ethyl acetate $1: 4 \mathrm{v} / \mathrm{v})=228-229^{\circ} \mathrm{C}$; IR $(\mathrm{KBr}) v_{\max }: 3094,2870,2816,1687,1620,1396,1103 \mathrm{~cm}^{-1}$; 
${ }^{1} \mathrm{H}$ NMR $\left(300 \mathrm{MHz}, \mathrm{CDCl}_{3}\right) \delta_{\mathrm{H}}: 2.33(\mathrm{~s}, 3 \mathrm{H}), 2.60(\mathrm{~d}, J=14.1 \mathrm{~Hz}, 1 \mathrm{H}), 2.79-2.94(\mathrm{~m}, 2 \mathrm{H})$, 3.39-3.57 (m, 3H), $4.80(\mathrm{~m}, 2 \mathrm{H}), 6.63-6.74(\mathrm{~m}, 3 \mathrm{H}), 6.87$ (d, J=7.8 Hz, 1H), 6.98-7.04 (m, 2H), $7.15(\mathrm{~d}, J=7.8 \mathrm{~Hz}, 2 \mathrm{H}), 7.31$ (d, $J=7.5 \mathrm{~Hz}, 2 \mathrm{H}), 7.61$ (br s, $1 \mathrm{H}), 8.00$ (d, $J=2.4 \mathrm{~Hz}, 1 \mathrm{H})$; ${ }^{13} \mathrm{C}$ NMR $\left(75 \mathrm{MHz}, \mathrm{CDCl}_{3}\right) \delta_{\mathrm{C}}: 20.4,31.6,33.1,44.8,49.7,64.4,67.3,69.2,109.4,120.7,124.3$, 126.2, 127.1, 128.8, 128.9, 129.4, 129.6, 130.9, 131.6, 132.6, 136.6, 139.7, 141.8, 176.1, 191.

\section{5-Chloro-7'-(4-chlorophenyl)-7',7a'-dihydro-1' H,3' H-dispiro[indoline-3,5'-pyrrolo[1,2-}

c]thiazole-6',3"-thiochromane]-2,4"-dione 5\{2,2,2\}: White solid. Yield: 80\%; mp (petroleum ether-ethyl acetate $1: 4 \mathrm{v} / \mathrm{v})=210-211^{\circ} \mathrm{C}$; IR $(\mathrm{KBr}) v_{\max }: 3174,2928,2827,1698,1667,1620$, 1584, $1479 \mathrm{~cm}^{-1} ;{ }^{1} \mathrm{H}$ NMR $\left(300 \mathrm{MHz}, \mathrm{CDCl}_{3}\right) \delta_{\mathrm{H}}: 2.61(\mathrm{~d}, J=14.1 \mathrm{~Hz}, 1 \mathrm{H}), 2.80(\mathrm{t}, J=8.8 \mathrm{~Hz}$, $1 \mathrm{H}), 2.90-2.95(\mathrm{~m}, 1 \mathrm{H}), 3.36-3.50(\mathrm{~m}, 3 \mathrm{H}), 4.73(\mathrm{dd}, J=14.5,8.8 \mathrm{~Hz}, 1 \mathrm{H}), 4.86(\mathrm{~d}, J=9.6 \mathrm{~Hz}$, $1 \mathrm{H}), 6.60(\mathrm{~d}, J=8.1 \mathrm{~Hz}, 1 \mathrm{H}), 6.77-6.81(\mathrm{~m}, 2 \mathrm{H}), 6.94(\mathrm{dd}, J=8.1,1.9 \mathrm{~Hz}, 1 \mathrm{H}), 7.05-7.14(\mathrm{~m}$, 2H), 7.32 (d, $J=7.5 \mathrm{~Hz}, 2 \mathrm{H}), 7.40$ (d, $J=7.5 \mathrm{~Hz}, 2 \mathrm{H}), 7.96($ br s, $1 \mathrm{H}), 8.10-8.12(\mathrm{~m}, 1 \mathrm{H}) ;{ }^{13} \mathrm{C}$ NMR $\left(75 \mathrm{MHz}, \mathrm{CDCl}_{3}\right) \delta_{\mathrm{C}}: 32.1,33.5,45.1,49.5,65.8,68.3,69.9,110.7,124.9,126.2,127.0$, 127.8, 129.0, 129.3, 130.4, 131.0, 132.9, 133.6, 134.8, 139.5, 140.9, 176.7, 191.9; ESI-MS: m/z. Calcd: 538.03;Found: $538.99\left(\mathrm{M}^{+}\right)$; Anal. Calcd for $\mathrm{C}_{27} \mathrm{H}_{20} \mathrm{Cl}_{2} \mathrm{~N}_{2} \mathrm{O}_{2} \mathrm{~S}_{2}$ : C, 60.11; $\mathrm{H}, 3.74 ; \mathrm{N}$, 5.19; \%. Found C, 59.70; H, 3.84; N, $5.25 \%$.

\section{5-Bromo-7'-(4-chlorophenyl)-7',7a'-dihydro-1' H,3' H-dispiro[indoline-3,5'-pyrrolo[1,2-}

c]thiazole-6',3"'-thiochromane]-2,4"'-dione 5\{2,3,2\}: White solid. Yield: 84\%; mp (petroleum ether-ethyl acetate $1: 4 \mathrm{v} / \mathrm{v})=198-199{ }^{\circ} \mathrm{C}$; IR $(\mathrm{KBr}) v_{\max }: 3125,3050,2841,1701,1672,1617$, 1587, $1476 \mathrm{~cm}^{-1} ;{ }^{1} \mathrm{H}$ NMR $\left(300 \mathrm{MHz}, \mathrm{CDCl}_{3}\right) \delta_{\mathrm{H}}: 2.60(\mathrm{~d}, J=14.4 \mathrm{~Hz}, 1 \mathrm{H}), 2.80(\mathrm{t}, J=8.8 \mathrm{~Hz}$, 1H), 2.90-2.94 (m, 1H), 3.36-3.50 (m, 3H), 4.73 (td, $J=9.2,5.3 \mathrm{~Hz}, 1 \mathrm{H}), 4.86(\mathrm{~d}, J=9.9 \mathrm{~Hz}$, $1 \mathrm{H}), 6.54(\mathrm{~d}, J=8.4 \mathrm{~Hz}, 1 \mathrm{H}), 6.76-6.79(\mathrm{~m}, 1 \mathrm{H}), 6.94(\mathrm{~d}, J=1.2 \mathrm{~Hz}, 1 \mathrm{H}), 7.06-7.14(\mathrm{~m}, 3 \mathrm{H})$, $7.32(\mathrm{~d}, J=8.7 \mathrm{~Hz}, 2 \mathrm{H}), 7.40(\mathrm{~d}, J=7.8 \mathrm{~Hz}, 2 \mathrm{H}), 7.66($ br s, $1 \mathrm{H}), 8.11-8.14(\mathrm{~m}, 1 \mathrm{H}) ;{ }^{13} \mathrm{C} \mathrm{NMR}$ $\left(75 \mathrm{MHz}, \mathrm{CDCl}_{3}\right) \delta_{\mathrm{C}}: 32.1,33.5,45.0,49.4,65.8,68.3,69.7,110.0,115.0,124.9,126.2,127.3$, 128.9, 130.5, 131.0, 132.1, 132.9, 133.6, 134.8, 139.8, 140.9, 176.1, 191.9; ESI-MS: m/z. Calcd: 581.98; Found: $583.11\left(\mathrm{M}^{+}\right)$. 


\section{Structure Elucidation of $4\{3,1,1\}$ by NMR}

The structure of the spirooxindole-pyrrolidine/pyrrolothiazole-thiochroman-4-one hybrids $\mathbf{4 / 5}$ was deduced from one- and two-dimensional NMR spectroscopic, microanalytical and mass spectral data, as detailed for $\mathbf{4}\{3,1,1\}$ as a representative example (Figure. A). The characteristic benzylic proton H-4' appears as a doublet of doublet at $5.14 \mathrm{ppm}(J=7.8$ and $10.5 \mathrm{~Hz})$, which shows HMBCs with C-3, C-2", C-3", C-4" at 74.9, 33.4, 59.9 and 193.3 respectively. The H-5' diasteretopic protons appears as a triplet and triplet at 3.41 and $3.91 \mathrm{ppm}(J=9.7 \mathrm{~Hz})$ and $(J=$ 8.4 Hz), which shows HMBCs with C-4' at $46.7 \mathrm{ppm}$. Likewise the C-2" diastereotopic protons appears as a doublet and doublet at $2.61(J=14.1 \mathrm{~Hz})$ and $3.32(J=14.1 \mathrm{~Hz}) \mathrm{ppm}$ which shows HMBCs with C-3, C-4', C-3", C-4" at 74.9, 46.7, 59.9 and 193.3 ppm respectively. The $N$-methyl protons appears as a singlet at 2.09 ppm which shows HMBCs with C-3 and C-5' at 74.9 and $56.7 \mathrm{ppm}$. The NH peak appears as a broad singlet at $7.79 \mathrm{ppm}$. ESI-Mass spectral studies, which show $\mathrm{M}+1$ and $\mathrm{M}+3$ peaks, also support the above structure.

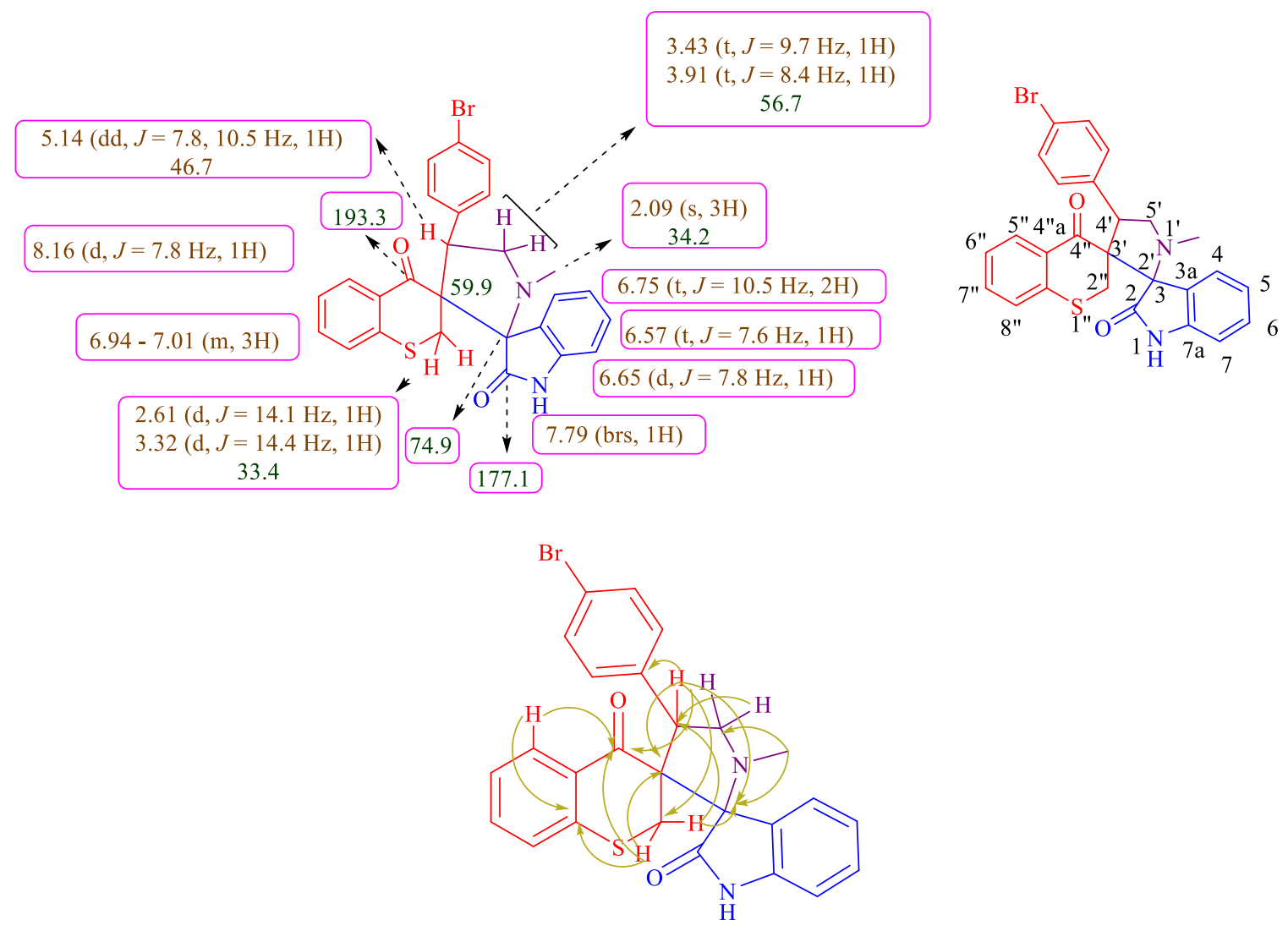

Figure A. Selected ${ }^{1} \mathrm{H}$ and ${ }^{13} \mathrm{C}$ Chemical shifts and HMB correlations of $4\{3,1,1\}$. 


\section{Copies of Spectra}

\begin{tabular}{|c|c|c|}
\hline No. & List of Figures & Page \\
\hline 1 & ${ }^{1} \mathrm{H}$ NMR Spectrum $4\{1,1,1\}$ & 18 \\
\hline 2 & ${ }^{13} \mathrm{C}$ NMR Spectrum of $\mathbf{4}\{1,1,1\}$ & 18 \\
\hline 3 & ESI mass spectrum of $\mathbf{4}\{1,1,1\}$ & 19 \\
\hline 4 & ${ }^{1} \mathrm{H}$ NMR Spectrum $\mathbf{4}\{2,1,1\}$ & 19 \\
\hline 5 & ${ }^{13} \mathrm{C}$ NMR Spectrum of $\mathbf{4}\{2,1,1\}$ & 20 \\
\hline 6 & ESI mass spectrum of $\mathbf{4}\{2,1,1\}$ & 20 \\
\hline 7 & ${ }^{1}$ H NMR Spectrum $\mathbf{4}\{3,1,1\}$ & 21 \\
\hline 8 & ${ }^{1} \mathrm{H}$ NMR Spectrum Expansion $4\{3,1,1\}$ & 21 \\
\hline 9 & ${ }^{13} \mathrm{C}$ NMR Spectrum of $\mathbf{4}\{3,1,1\}$ & 22 \\
\hline 10 & DEPT Spectrum of $\mathbf{4}\{3,1,1\}$ & 22 \\
\hline 11 & $\mathrm{H}, \mathrm{H}-\mathrm{COSY}$ Spectrum of $\mathbf{4}\{3,1,1\}$ & 23 \\
\hline 12 & HMBC Spectrum of $4\{3,1,1\}$ & 23 \\
\hline 12 & ESI mass spectrum of $\mathbf{4}\{3,1,1\}$ & 24 \\
\hline 14 & ${ }^{1} \mathrm{H}$ NMR Spectrum $4\{4,1,1\}$ & 24 \\
\hline 15 & ${ }^{13} \mathrm{C}$ NMR Spectrum of $\mathbf{4}\{4,1,1\}$ & 25 \\
\hline 16 & ESI mass spectrum of $\mathbf{4}\{4,1,1\}$ & 25 \\
\hline 17 & ${ }^{1} \mathrm{H}$ NMR Spectrum $\mathbf{4}\{5,1,1\}$ & 26 \\
\hline 18 & ${ }^{13} \mathrm{C}$ NMR Spectrum of $\mathbf{4}\{5,1,1\}$ & 26 \\
\hline 19 & ESI mass spectrum of $\mathbf{4}\{5,1,1\}$ & 27 \\
\hline 20 & ${ }^{1} \mathrm{H}$ NMR Spectrum $\mathbf{4}\{7,1,1\}$ & 27 \\
\hline 21 & ${ }^{13} \mathrm{C}$ NMR Spectrum of $\mathbf{4}\{7,1,1\}$ & 28 \\
\hline 22 & ESI mass spectrum of $\mathbf{4}\{7,1,1\}$ & 28 \\
\hline 23 & ${ }^{1} \mathrm{H}$ NMR Spectrum $\mathbf{4}\{8,1,1\}$ & 29 \\
\hline 24 & ${ }^{13} \mathrm{C}$ NMR Spectrum of $\mathbf{4}\{8,1,1\}$ & 29 \\
\hline 25 & ESI mass spectrum of $\mathbf{4}\{8,1,1\}$ & 30 \\
\hline 26 & ${ }^{1} \mathrm{H}$ NMR Spectrum $\mathbf{4}\{9,1,1\}$ & 30 \\
\hline
\end{tabular}




\begin{tabular}{|c|c|c|}
\hline No. & List of Figures & Page \\
\hline 27 & ${ }^{13} \mathrm{C}$ NMR Spectrum of $\mathbf{4}\{9,1,1\}$ & 31 \\
\hline 28 & ESI mass spectrum of $\mathbf{4}\{9,1,1\}$ & 31 \\
\hline 29 & ${ }^{1} \mathrm{H}$ NMR Spectrum $4\{12,1,1\}$ & 32 \\
\hline 30 & ${ }^{13} \mathrm{C}$ NMR Spectrum of $\mathbf{4}\{12,1,1\}$ & 32 \\
\hline 31 & ESI mass spectrum of $\mathbf{4}\{12,1,1\}$ & 33 \\
\hline 32 & ${ }^{1} \mathrm{H}$ NMR Spectrum $4\{13,1,1\}$ & 33 \\
\hline 33 & ${ }^{13} \mathrm{C}$ NMR Spectrum of $\mathbf{4}\{13,1,1\}$ & 34 \\
\hline 34 & ${ }^{1} \mathrm{H}$ NMR Spectrum $\mathbf{4}\{14,1,1\}$ & 34 \\
\hline 35 & ${ }^{13} \mathrm{C}$ NMR Spectrum of $\mathbf{4}\{14,1,1\}$ & 35 \\
\hline 36 & ${ }^{1} \mathrm{H}$ NMR Spectrum $\mathbf{4}\{2,2,1\}$ & 35 \\
\hline 37 & ${ }^{13} \mathrm{C}$ NMR Spectrum of $\mathbf{4}\{2,2,1\}$ & 36 \\
\hline 38 & ESI mass spectrum of $\mathbf{4}\{2,2,1\}$ & 36 \\
\hline 39 & ${ }^{1} \mathrm{H}$ NMR Spectrum $\mathbf{4}\{2,3,1\}$ & 37 \\
\hline 40 & ${ }^{13} \mathrm{C}$ NMR Spectrum of $\mathbf{4}\{2,3,1\}$ & 37 \\
\hline 41 & ESI mass spectrum of $\mathbf{4}\{2,3,1\}$ & 38 \\
\hline 42 & ${ }^{1} \mathrm{H}$ NMR Spectrum $\mathbf{5}\{1,1,2\}$ & 38 \\
\hline 43 & ${ }^{13} \mathrm{C}$ NMR Spectrum of $\mathbf{5}\{1,1,2\}$ & 39 \\
\hline 44 & ESI mass spectrum of $\mathbf{5}\{1,1,2\}$ & 39 \\
\hline 45 & ${ }^{1} \mathrm{H}$ NMR Spectrum $\mathbf{5}\{2,1,2\}$ & 40 \\
\hline 46 & ${ }^{13} \mathrm{C}$ NMR Spectrum of $\mathbf{5}\{2,1,2\}$ & 40 \\
\hline 47 & ESI mass spectrum of $\mathbf{5}\{2,1,2\}$ & 41 \\
\hline 48 & ${ }^{1} \mathrm{H}$ NMR Spectrum $\mathbf{5}\{3,1,2\}$ & 41 \\
\hline 49 & ${ }^{13} \mathrm{C}$ NMR Spectrum of $\mathbf{5}\{3,1,2\}$ & 42 \\
\hline 50 & ESI mass spectrum of $\mathbf{5}\{3,1,2\}$ & 42 \\
\hline
\end{tabular}




\begin{tabular}{|c|c|c|}
\hline No. & List of Figures & Page \\
\hline 51 & ${ }^{1} \mathrm{H}$ NMR Spectrum $\mathbf{5}\{4,1,2\}$ & 43 \\
\hline 52 & ${ }^{13} \mathrm{C}$ NMR Spectrum of $\mathbf{5}\{4,1,2\}$ & 43 \\
\hline 53 & ESI mass spectrum of $\mathbf{5}\{4,1,2\}$ & 44 \\
\hline 54 & ${ }^{1} \mathrm{H}$ NMR Spectrum $\mathbf{5}\{5,1,2\}$ & 44 \\
\hline 55 & ${ }^{13} \mathrm{C}$ NMR Spectrum of $5\{5,1,2\}$ & 45 \\
\hline 56 & ESI mass spectrum of $\mathbf{5}\{5,1,2\}$ & 45 \\
\hline 57 & ${ }^{1} \mathrm{H}$ NMR Spectrum $\mathbf{5}\{6,1,2\}$ & 46 \\
\hline 58 & ${ }^{13} \mathrm{C}$ NMR Spectrum of $\mathbf{5}\{6,1,2\}$ & 46 \\
\hline 59 & ESI mass spectrum of $\mathbf{5}\{6,1,2\}$ & 47 \\
\hline 60 & ${ }^{1} \mathrm{H}$ NMR Spectrum $\mathbf{5}\{7,1,2\}$ & 47 \\
\hline 61 & ${ }^{13} \mathrm{C}$ NMR Spectrum of $\mathbf{5}\{7,1,2\}$ & 48 \\
\hline 62 & ESI mass spectrum of $\mathbf{5}\{7,1,2\}$ & 48 \\
\hline 63 & ${ }^{1} \mathrm{H}$ NMR Spectrum $\mathbf{5}\{8,1,2\}$ & 49 \\
\hline 64 & ${ }^{13} \mathrm{C}$ NMR Spectrum of $\mathbf{5}\{8,1,2\}$ & 49 \\
\hline 65 & ESI mass spectrum of $\mathbf{5}\{8,1,2\}$ & 50 \\
\hline 66 & ${ }^{1} \mathrm{H}$ NMR Spectrum $\mathbf{5}\{9,1,2\}$ & 50 \\
\hline 67 & ${ }^{13} \mathrm{C}$ NMR Spectrum of $\mathbf{5}\{9,1,2\}$ & 51 \\
\hline 68 & ESI mass spectrum of $\mathbf{5}\{9,1,2\}$ & 51 \\
\hline 69 & ${ }^{1} \mathrm{H}$ NMR Spectrum $\mathbf{5}\{10,1,2\}$ & 52 \\
\hline 70 & ${ }^{13} \mathrm{C}$ NMR Spectrum of $\mathbf{5}\{10,1,2\}$ & 52 \\
\hline 71 & ESI mass spectrum of $\mathbf{5}\{10,1,2\}$ & 53 \\
\hline 72 & ${ }^{1} \mathrm{H}$ NMR Spectrum $\mathbf{5}\{11,1,2\}$ & 53 \\
\hline 73 & ${ }^{13} \mathrm{C}$ NMR Spectrum of $\mathbf{5}\{11,1,2\}$ & 54 \\
\hline 74 & ESI mass spectrum of $\mathbf{5}\{11,1,2\}$ & 54 \\
\hline
\end{tabular}




\begin{tabular}{|c|l|c|}
\hline No. & List of Figures & Page \\
\hline 75 & ${ }^{1} \mathrm{H}$ NMR Spectrum $\mathbf{5}\{12,1,2\}$ & 55 \\
\hline 76 & ${ }^{13} \mathrm{C}$ NMR Spectrum of $\mathbf{5}\{12,1,2\}$ & 56 \\
\hline 77 & ESI mass spectrum of $\mathbf{5}\{12,1,2\}$ & 56 \\
\hline 78 & ${ }^{1} \mathrm{H}$ NMR Spectrum $\mathbf{5}\{13,1,2\}$ & 57 \\
\hline 79 & ${ }^{13} \mathrm{C}$ NMR Spectrum of $\mathbf{5}\{13,1,2\}$ & 57 \\
\hline 80 & ${ }^{1} \mathrm{H}$ NMR Spectrum $\mathbf{5}\{15,1,2\}$ & 58 \\
\hline 81 & ${ }^{13} \mathrm{C}$ NMR Spectrum of $\mathbf{5}\{15,1,2\}$ & 58 \\
\hline 82 & ${ }^{1} \mathrm{H}$ NMR Spectrum $\mathbf{5}\{16,1,2\}$ & 59 \\
\hline 83 & ${ }^{13} \mathrm{C}$ NMR Spectrum of $\mathbf{5}\{16,1,2\}$ & 59 \\
\hline 84 & ${ }^{1} \mathrm{H}$ NMR Spectrum $\mathbf{5}\{2,2,2\}$ & 60 \\
\hline 85 & ${ }^{13} \mathrm{C}$ NMR Spectrum of $\mathbf{5}\{2,2,2\}$ & 60 \\
\hline 86 & ESI mass spectrum of $\mathbf{5}\{2,2,2\}$ & 61 \\
\hline 87 & ${ }^{1} \mathrm{H}$ NMR Spectrum $\mathbf{5}\{2,3,2\}$ & 61 \\
\hline 88 & ${ }^{13} \mathrm{C}$ NMR Spectrum of $\mathbf{5}\{2,3,2\}$ & 62 \\
\hline 89 & ESI mass spectrum of $\mathbf{5}\{2,3,2\}$ & \\
\hline
\end{tabular}




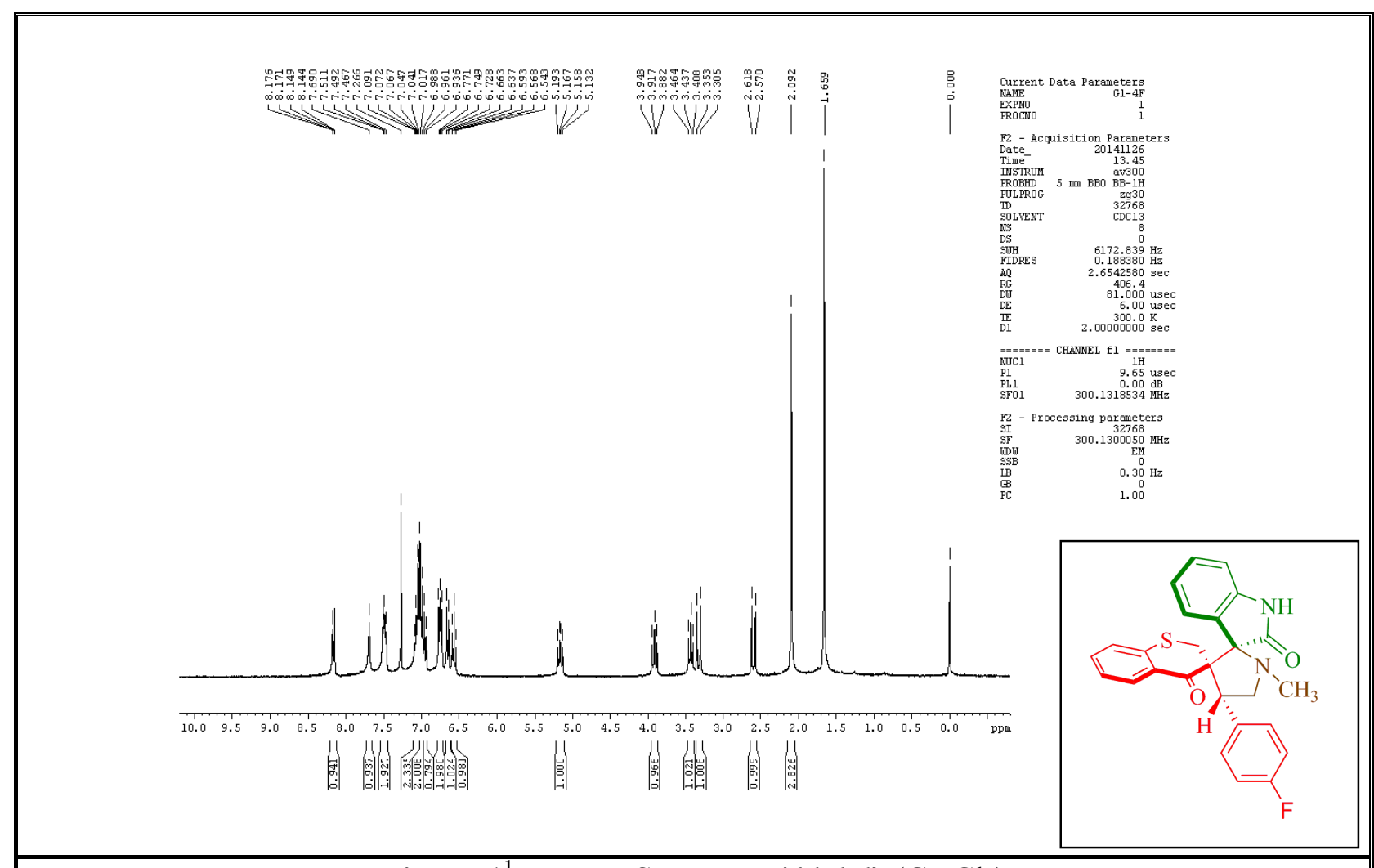

Figure. $1^{1} \mathrm{H}$ NMR Spectrum $4\{1,1,1\}\left(\mathrm{CDCl}_{3}\right)$ 


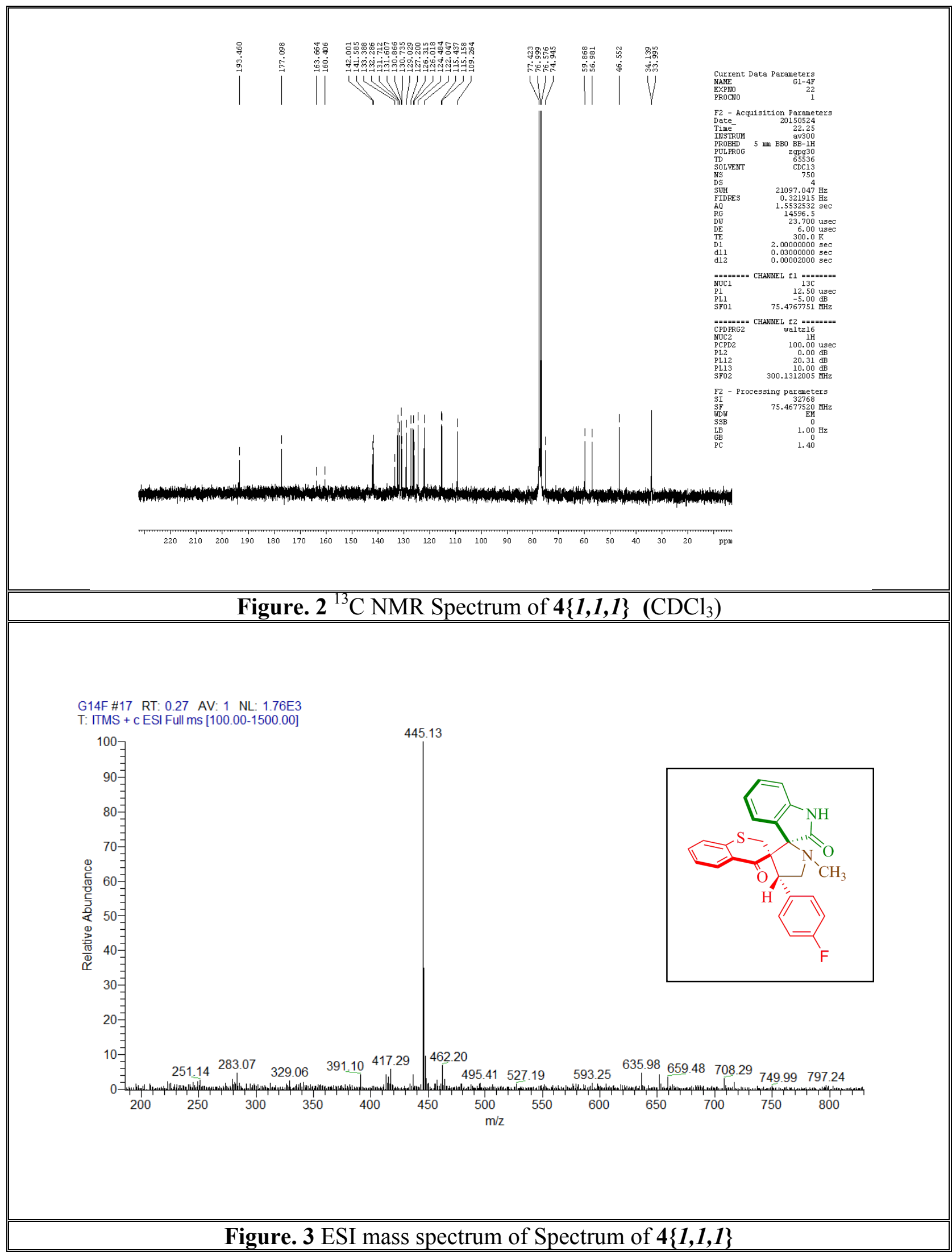




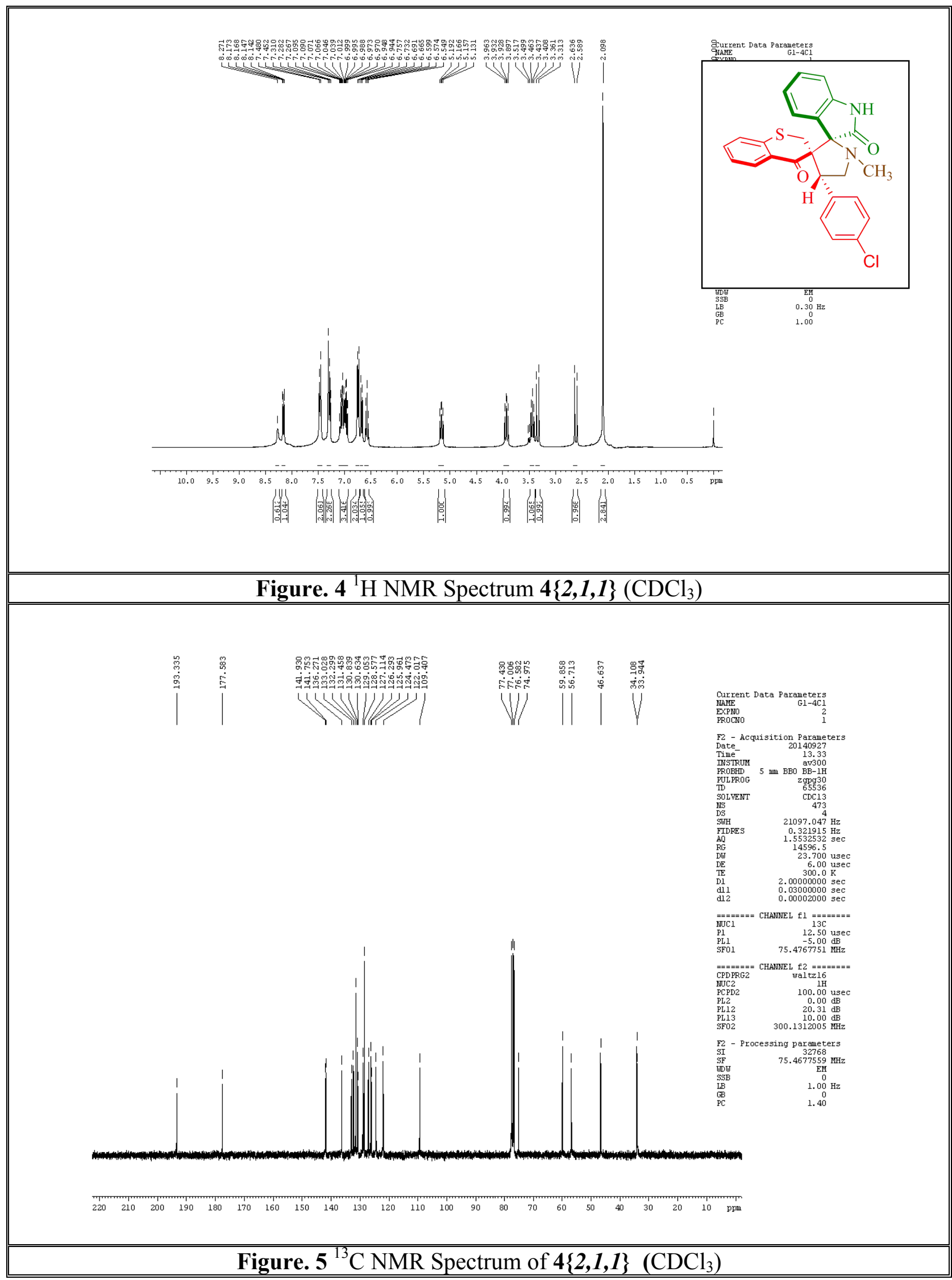




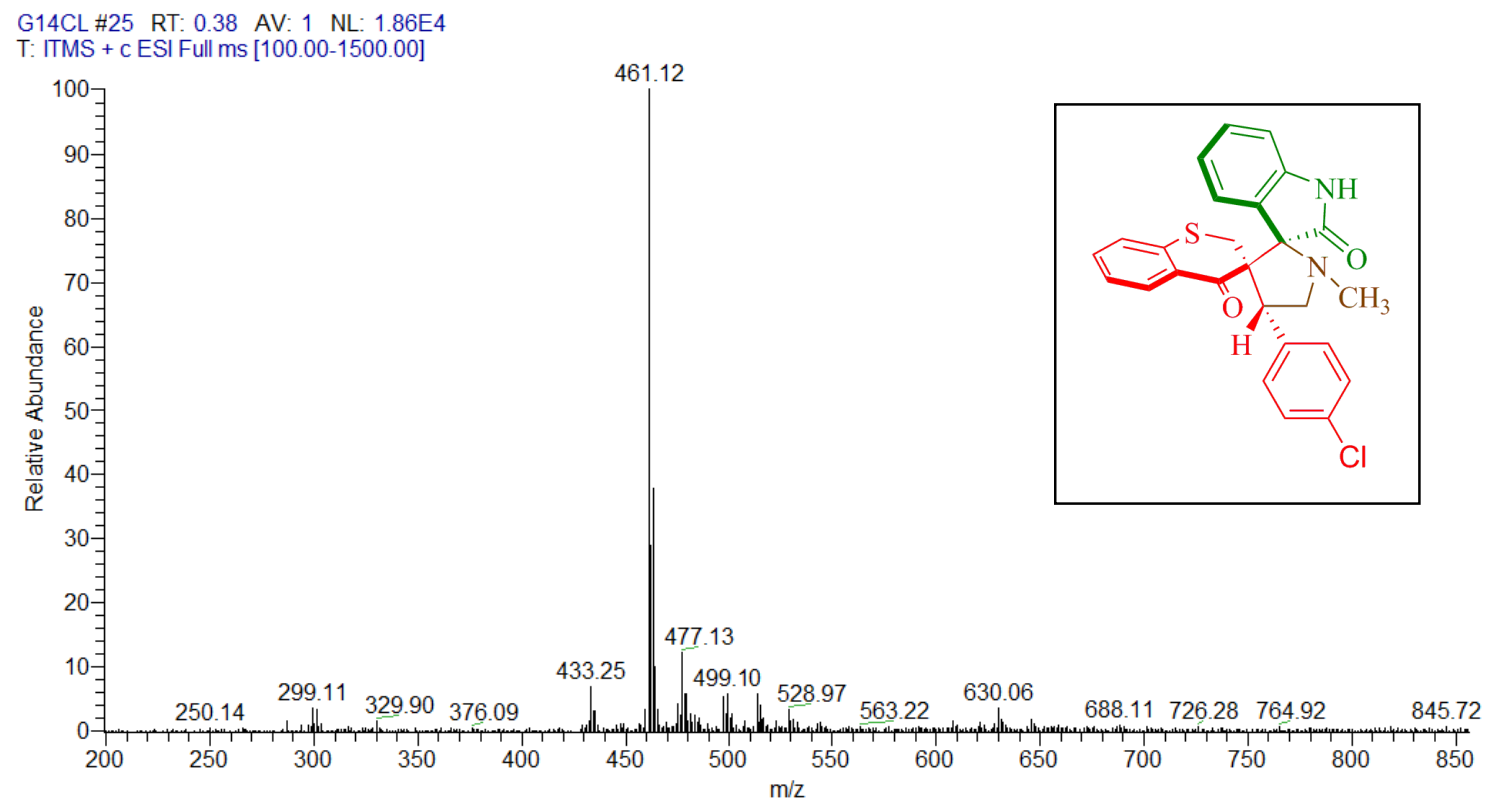

Figure. 6 ESI mass spectrum of Spectrum of $4\{2,1,1\}$

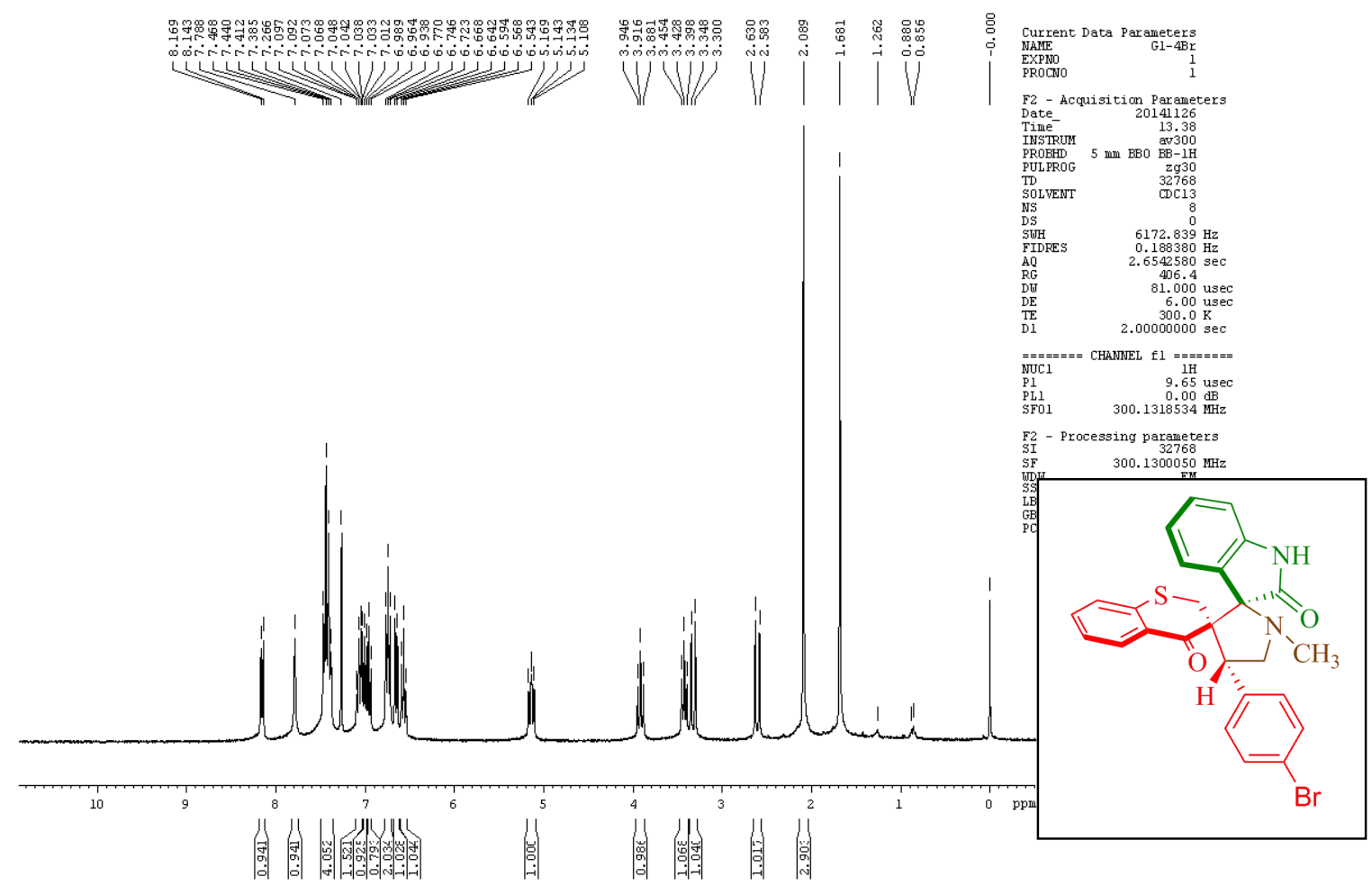

Figure. $7{ }^{1} \mathrm{H}$ NMR Spectrum $4\{3,1,1\}\left(\mathrm{CDCl}_{3}\right)$ 


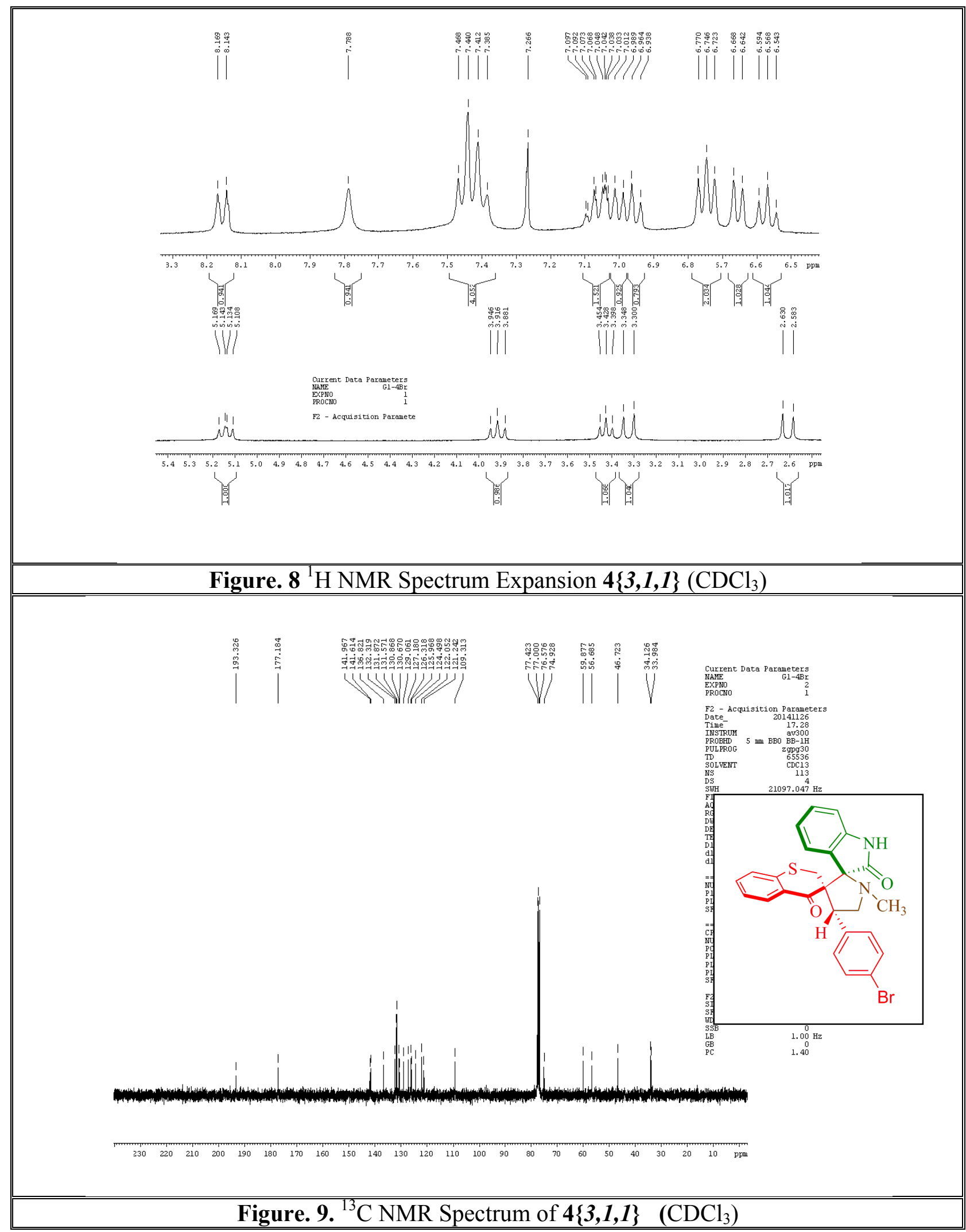




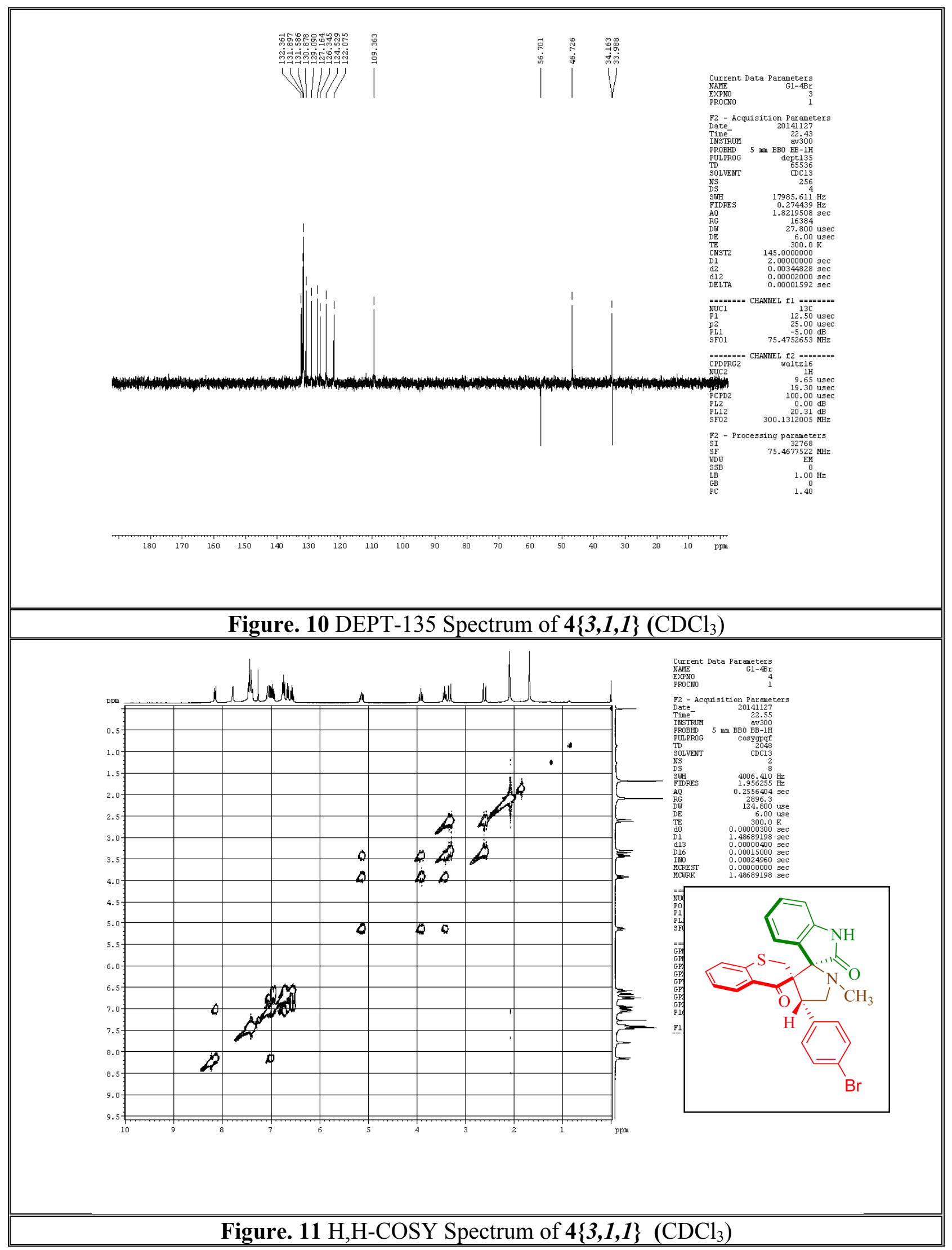



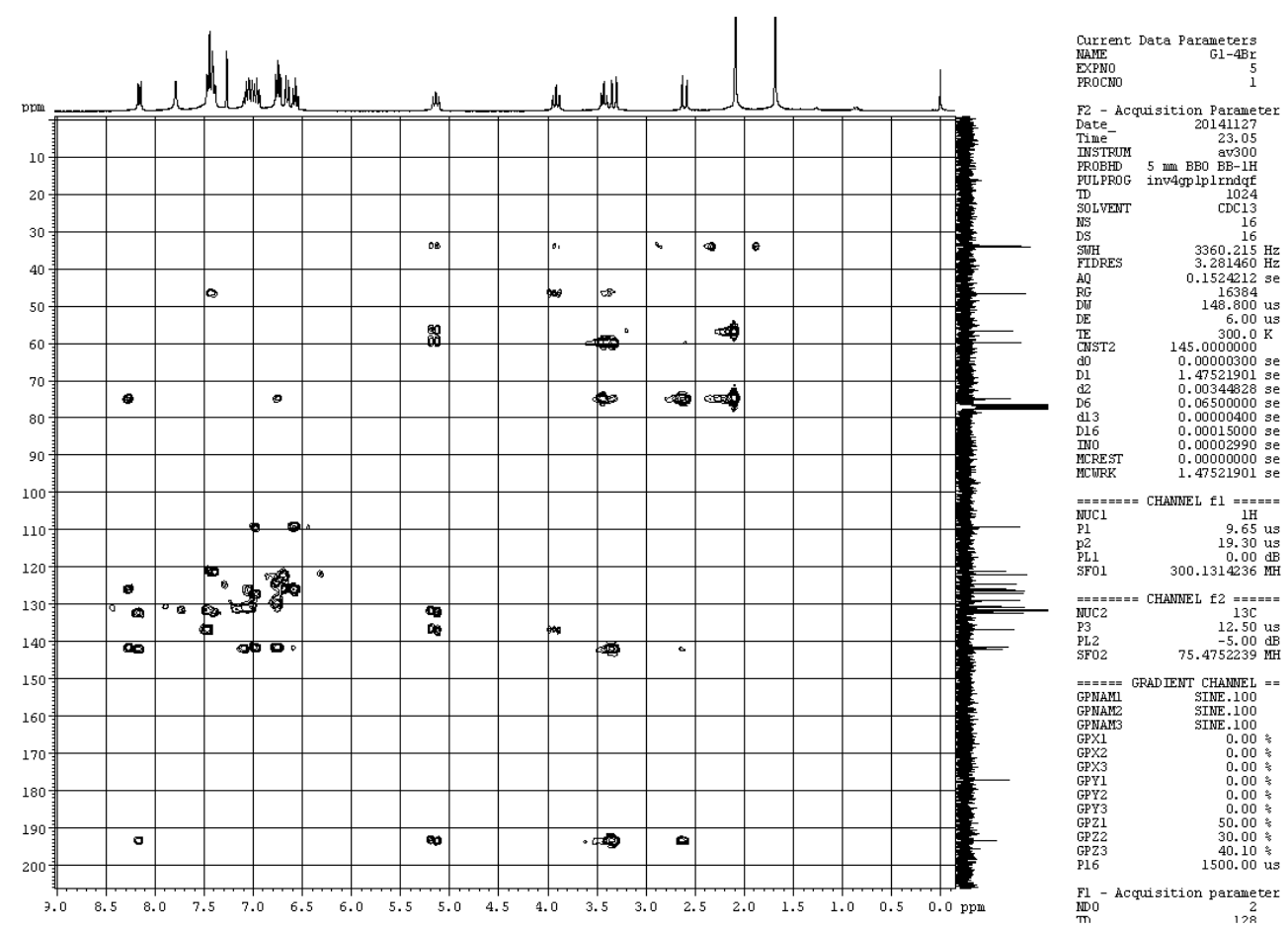

Figure. $12 \mathrm{HMBC}$ Spectrum of $4\{3,1,1\} \quad\left(\mathrm{CDCl}_{3}\right)$

G14BR \#23 RT: 0.37 AV: 1 NL: $1.52 E 3$

T: ITMS + c ESI Full ms [100.00-1500.00]

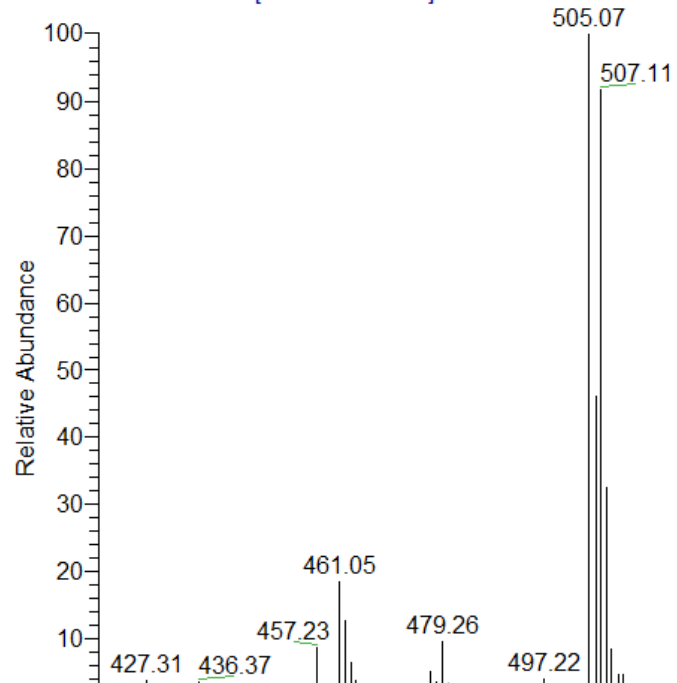

07.11

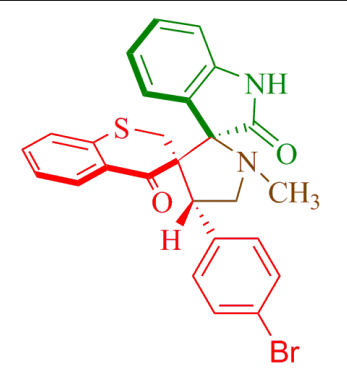

Figure. 13 ESI mass spectrum of Spectrum of $4\{3,1,1\}$ 


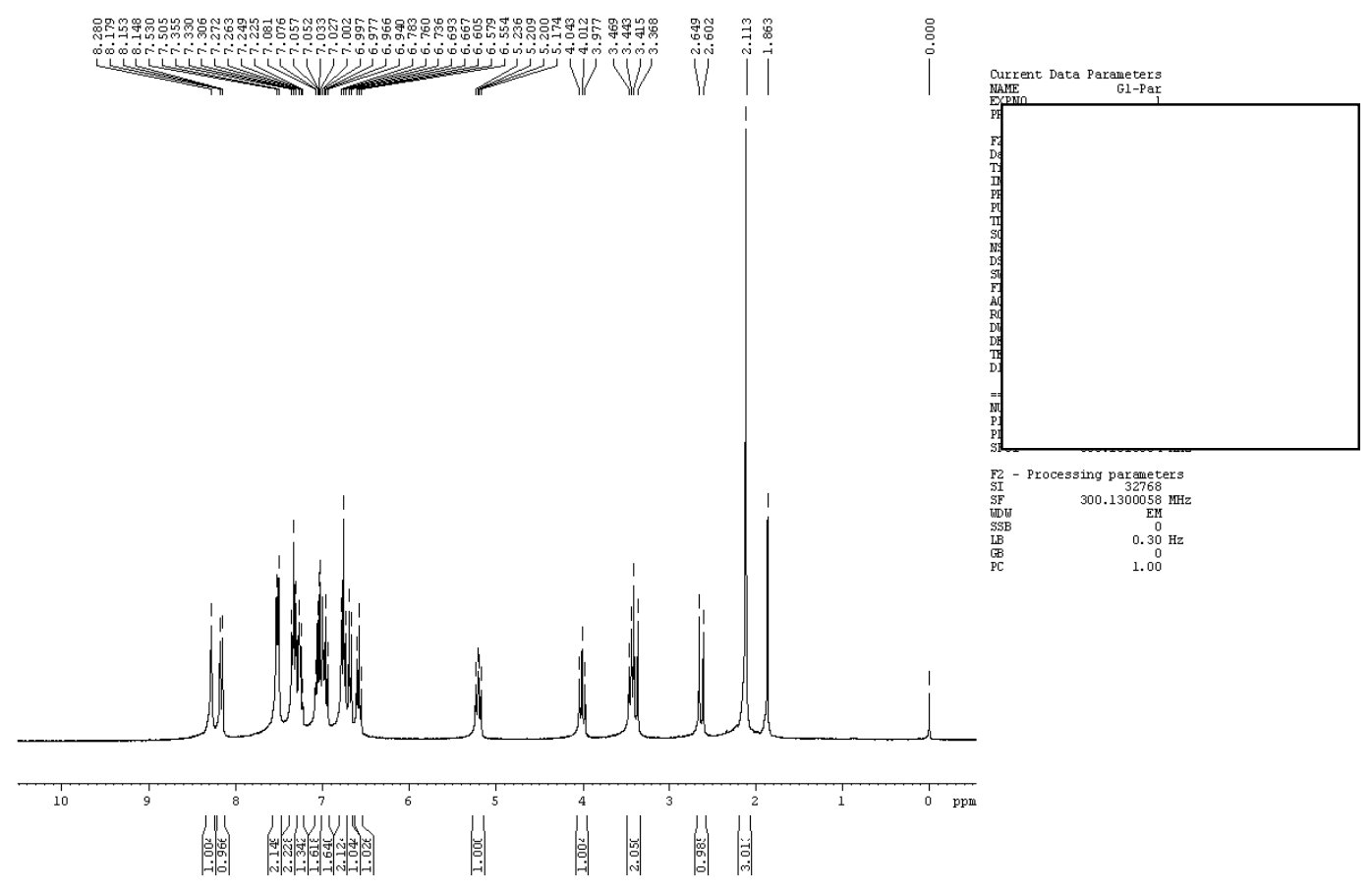

Figure. $14{ }^{1} \mathrm{H}$ NMR Spectrum 4\{4,1,1\} $\left(\mathrm{CDCl}_{3}\right)$

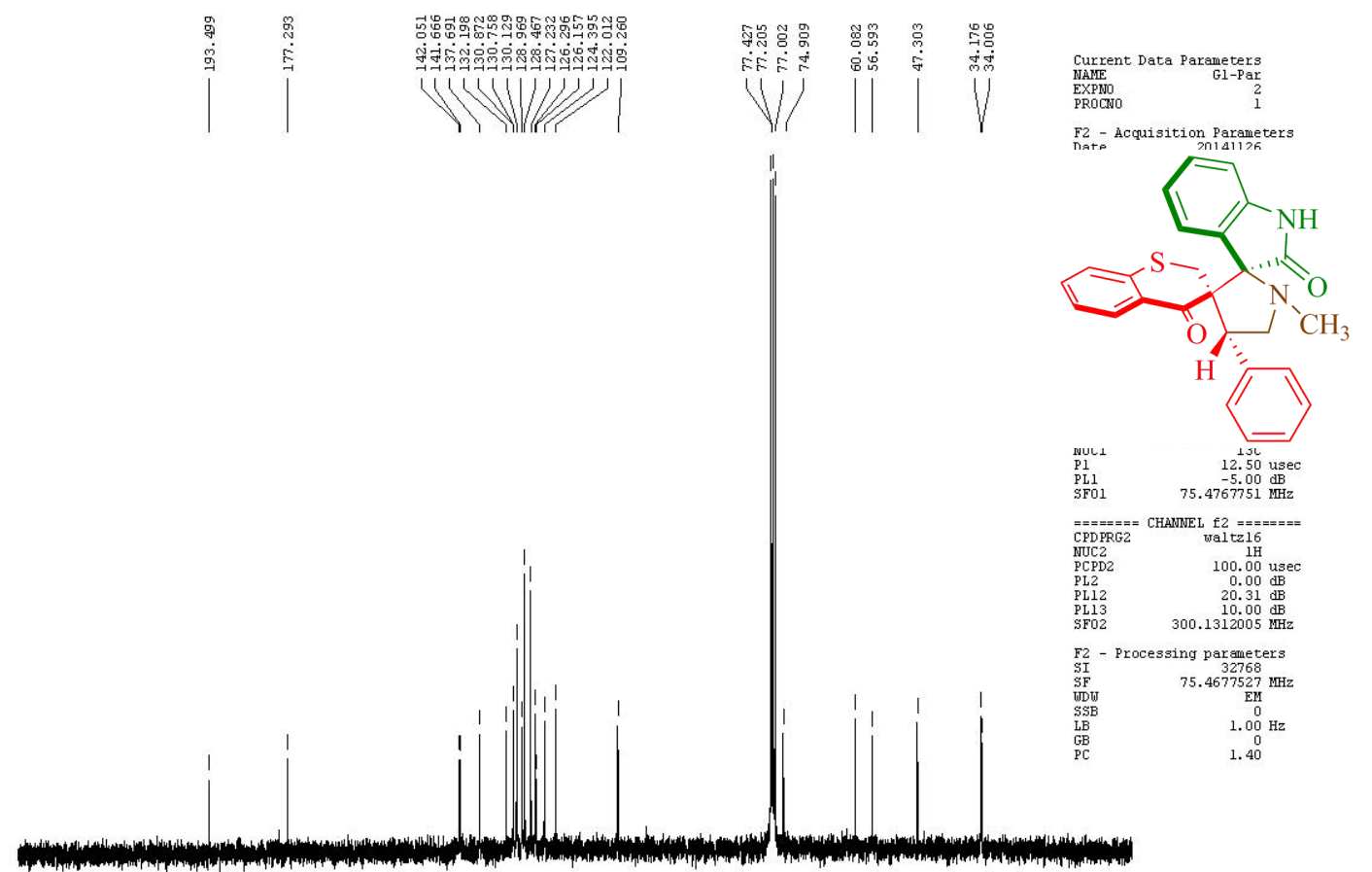

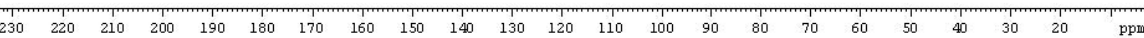


Figure. $15{ }^{13} \mathrm{C}$ NMR Spectrum of $4\{4,1,1\}\left(\mathrm{CDCl}_{3}\right)$

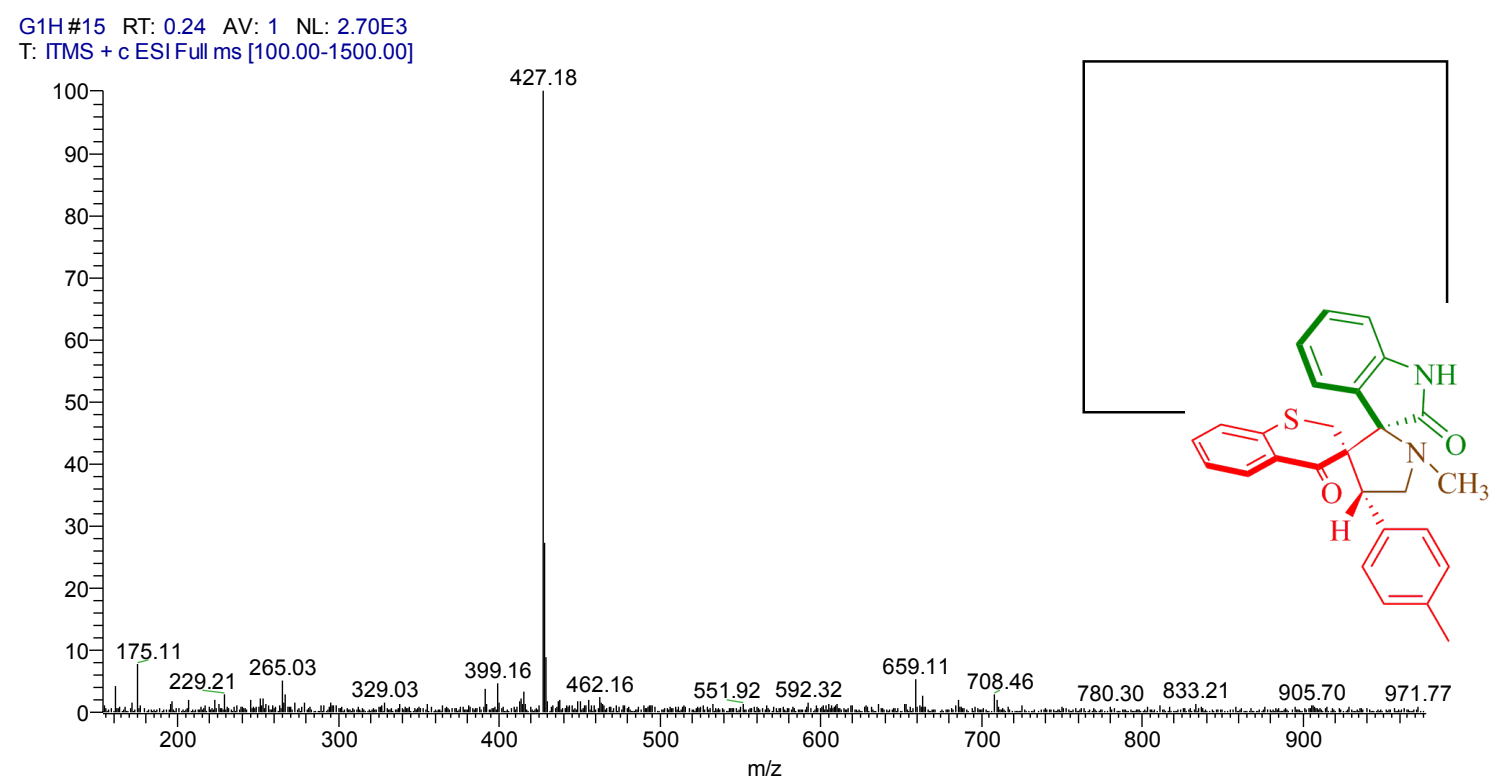

Figure. 16 ESI mass spectrum of Spectrum of $4\{4,1,1\}$

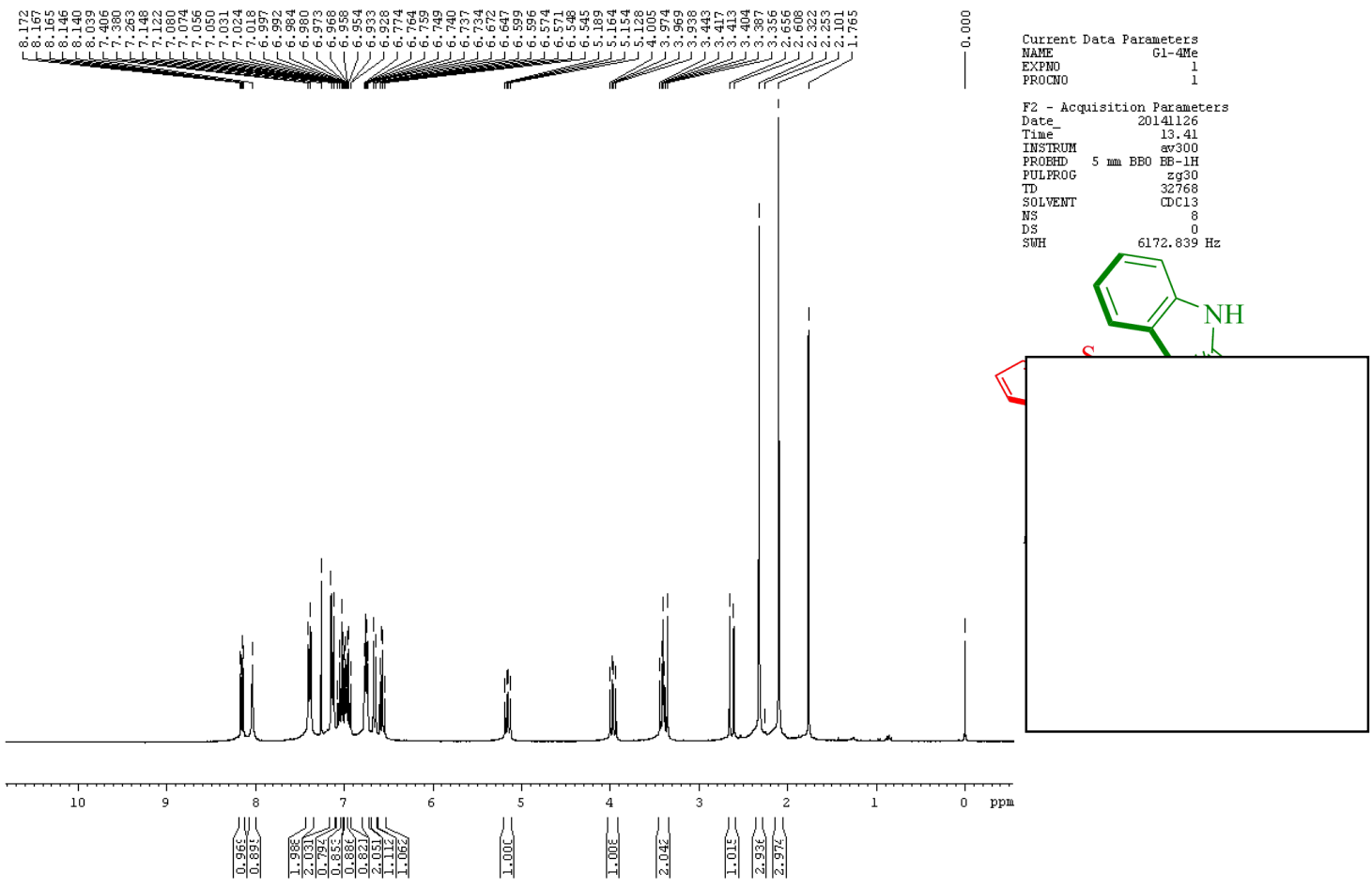

Figure. $17{ }^{1} \mathrm{H}$ NMR Spectrum $4\{5,1,1\} \quad\left(\mathrm{CDCl}_{3}\right)$ 


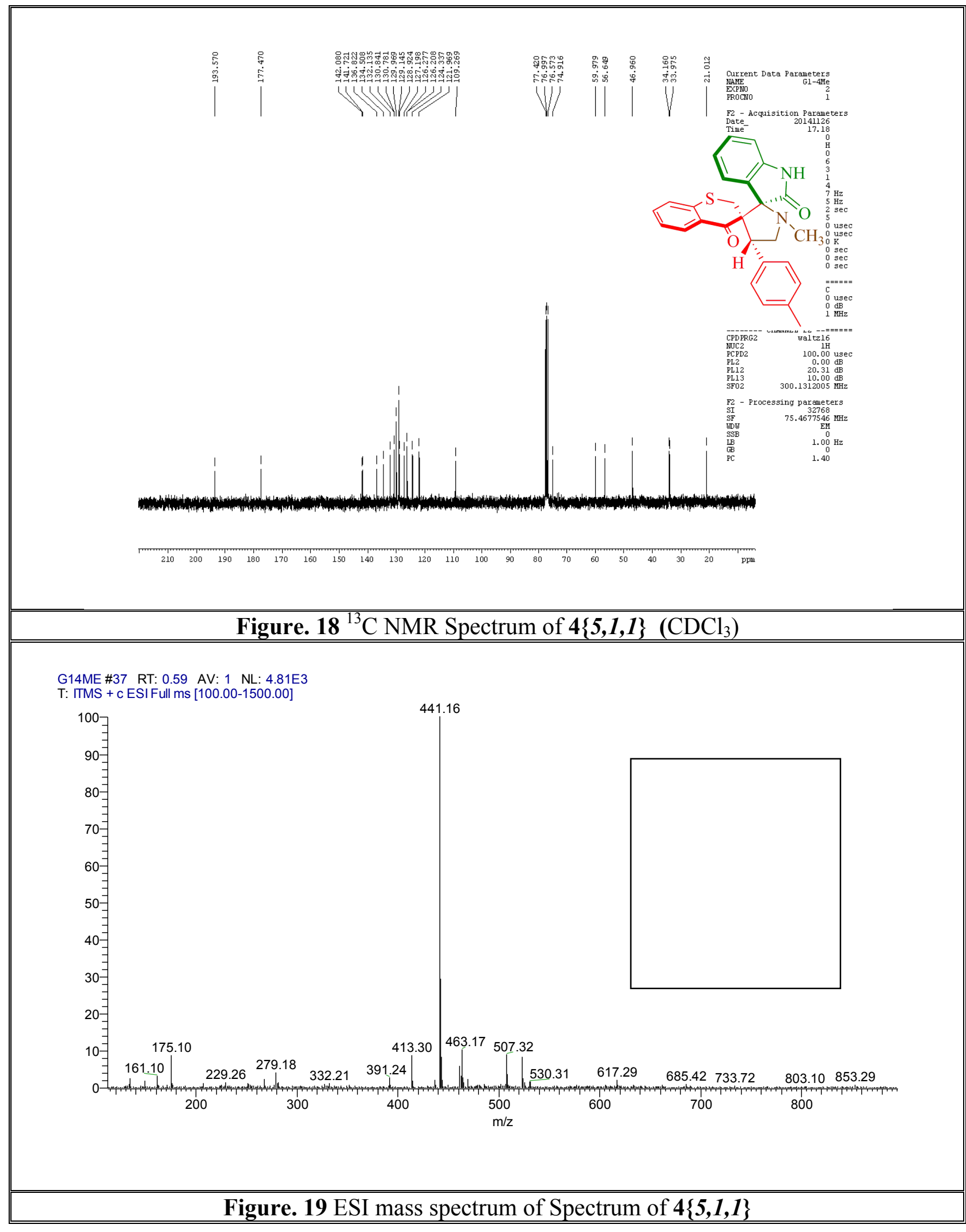




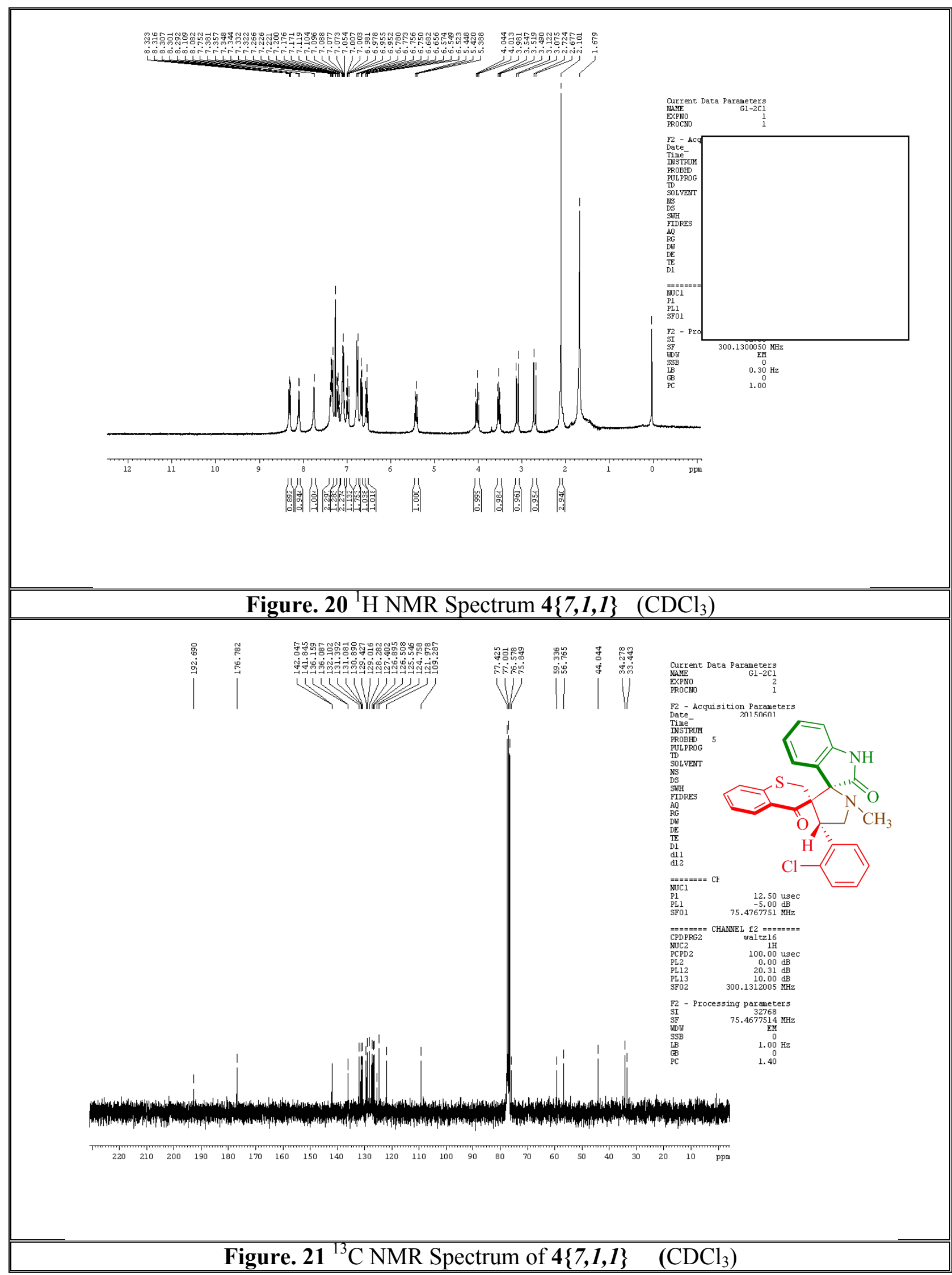


G-12CL \#16 RT: 0.24 AV: 1 NL: 7.58E3

T: ITMS - c ESI Full ms [100.00-2000.00]

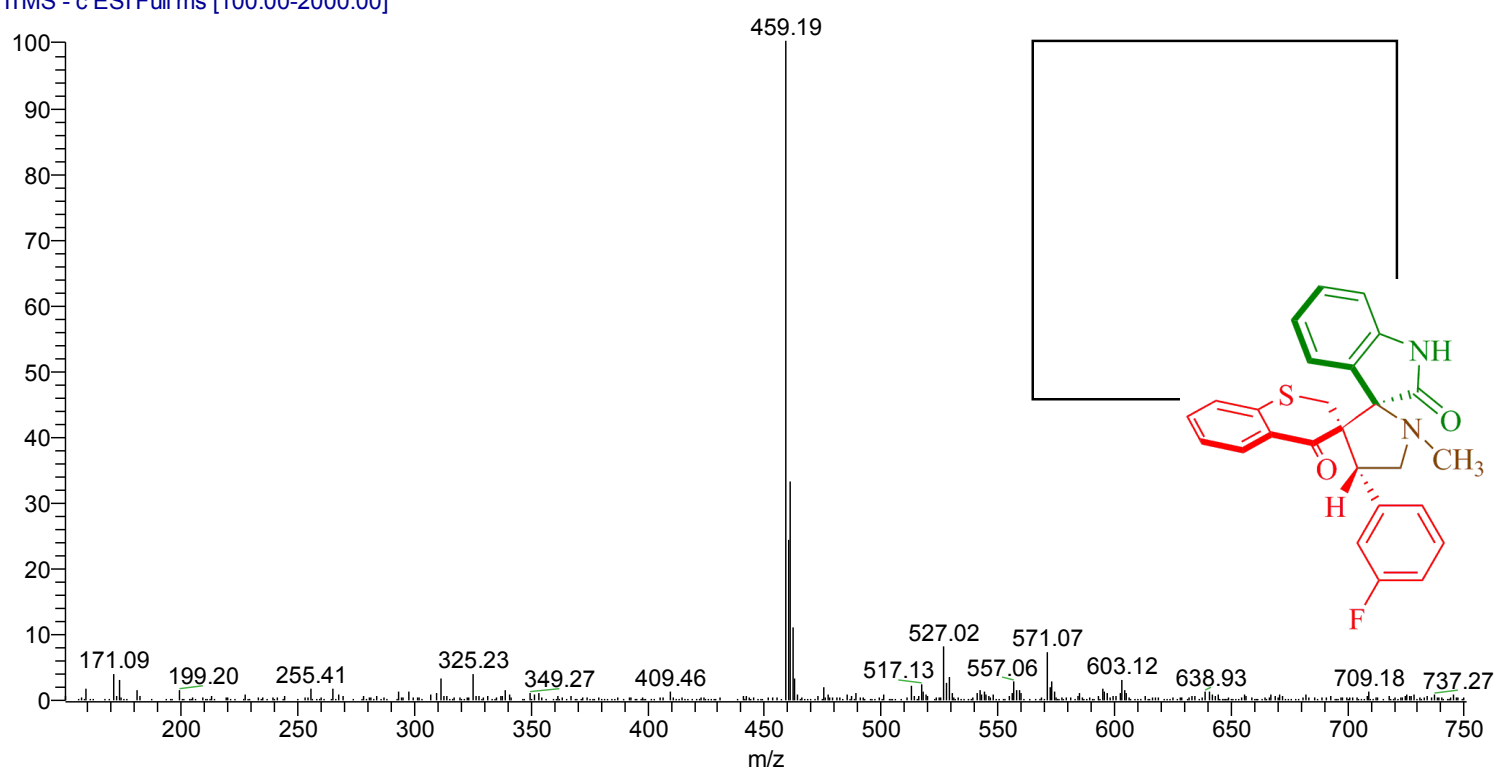

Figure. 22 ESI mass spectrum of Spectrum of $4\{7,1,1\}$

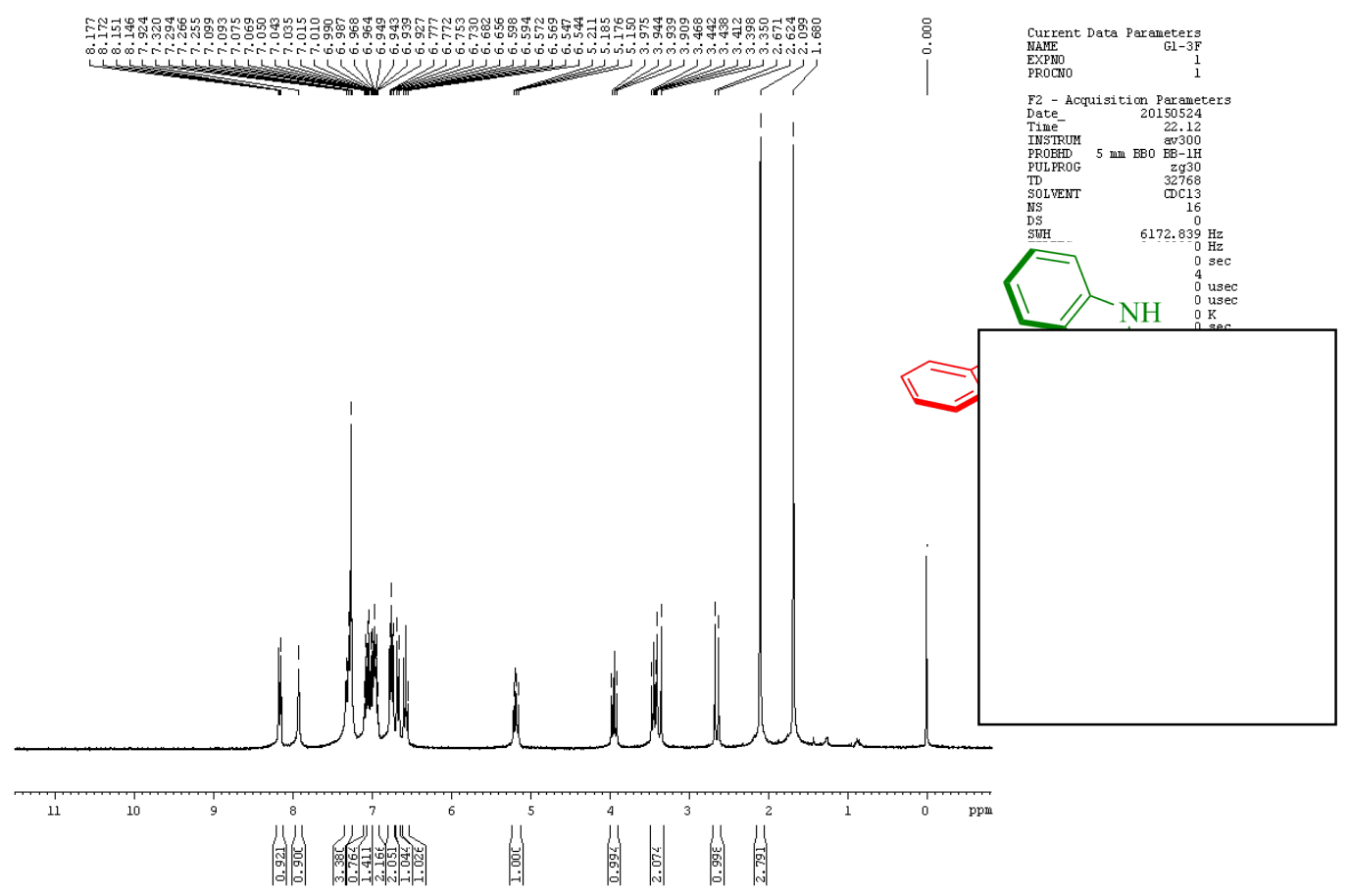

Figure. $23{ }^{1} \mathrm{H}$ NMR Spectrum $4\{8,1,1\} \quad\left(\mathrm{CDCl}_{3}\right)$ 


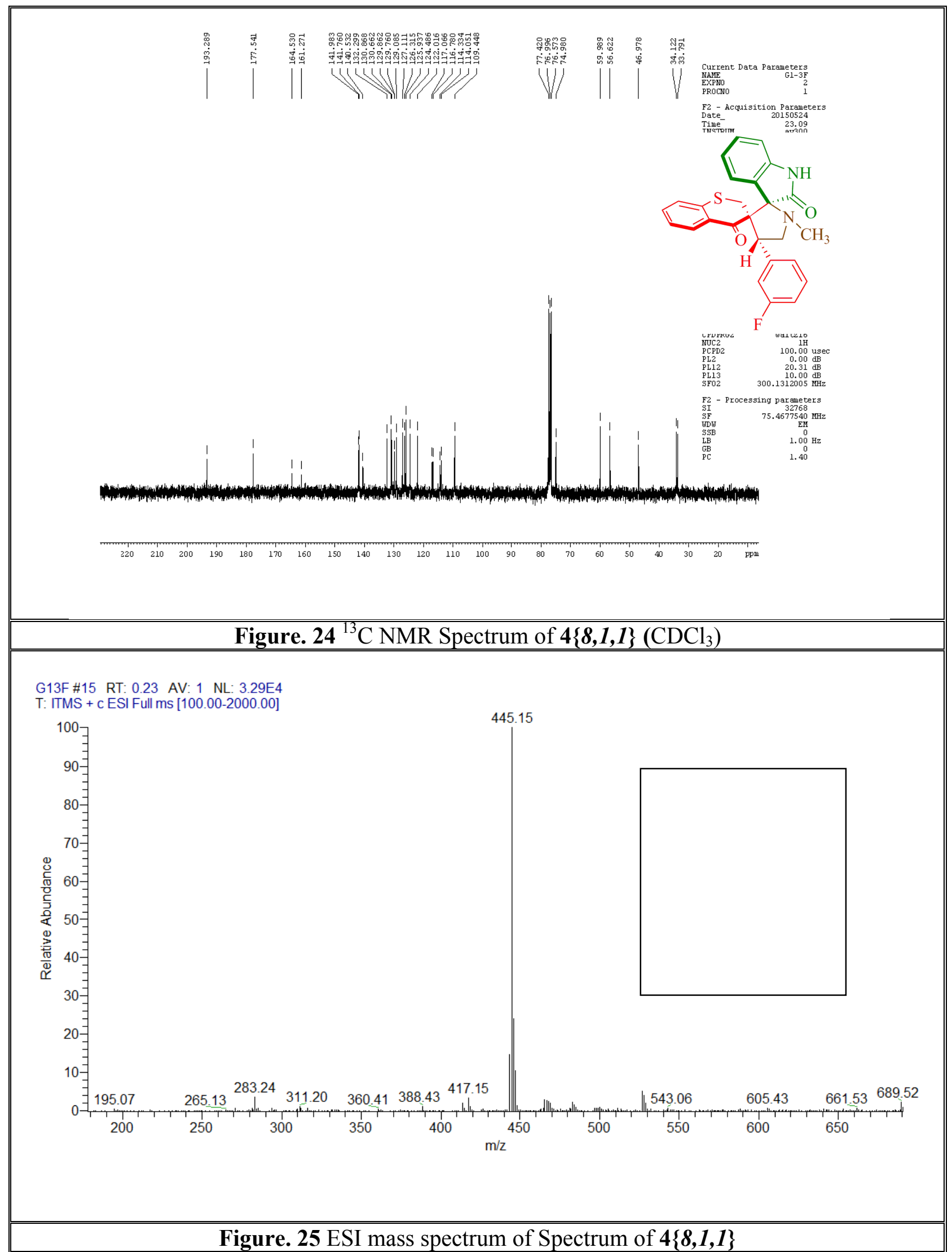

Figure. 25 ESI mass spectrum of Spectrum of $4\{8,1,1\}$ 


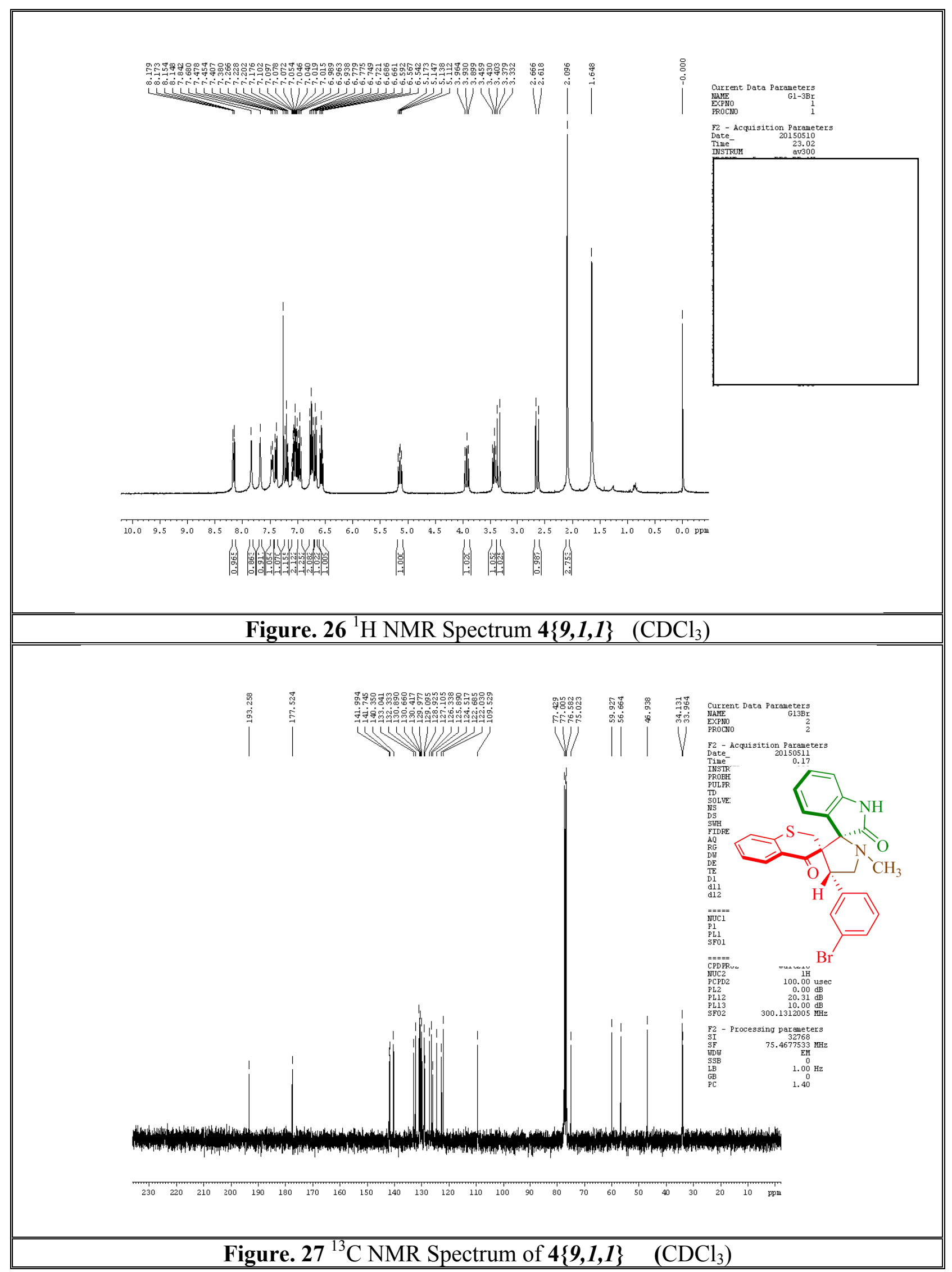




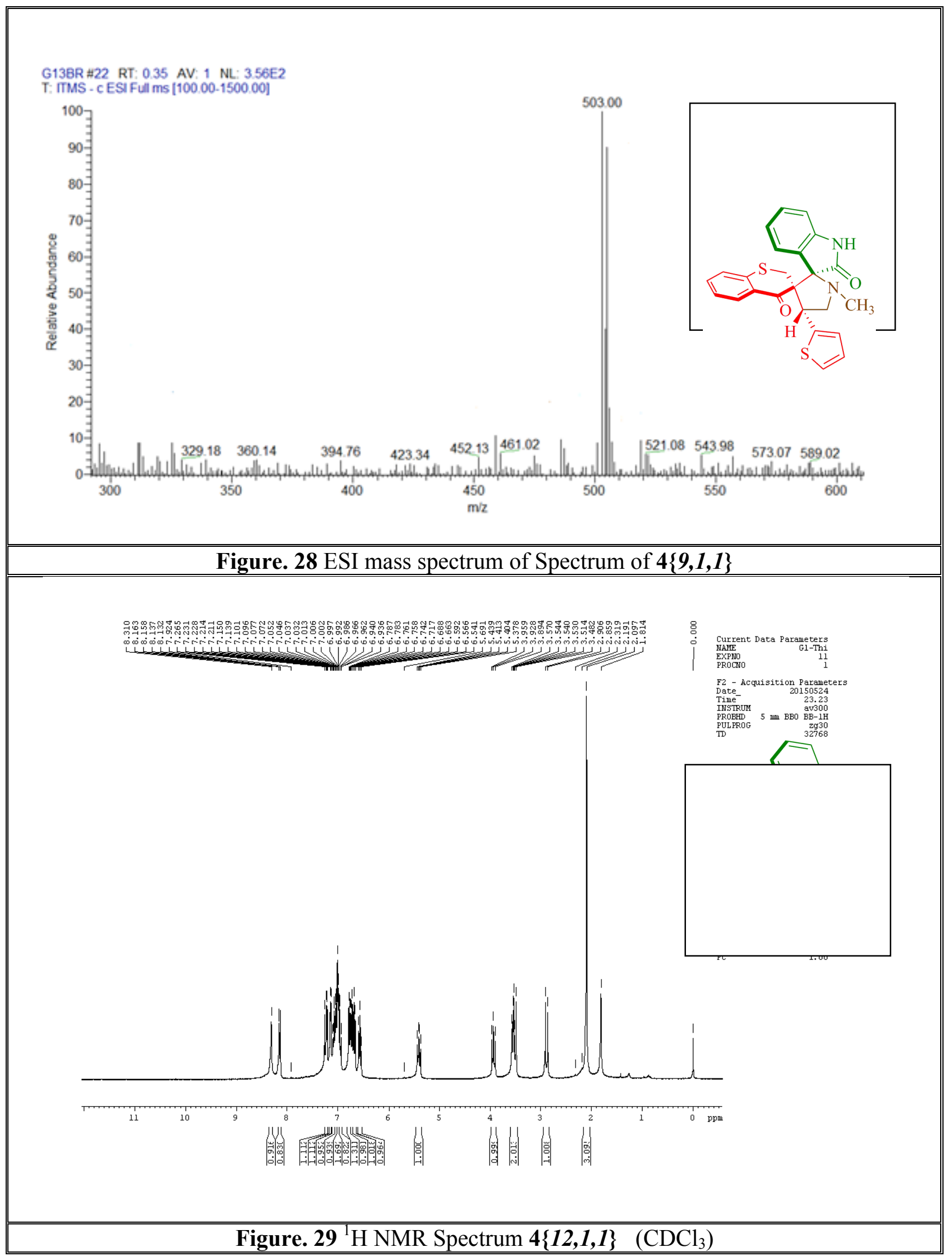




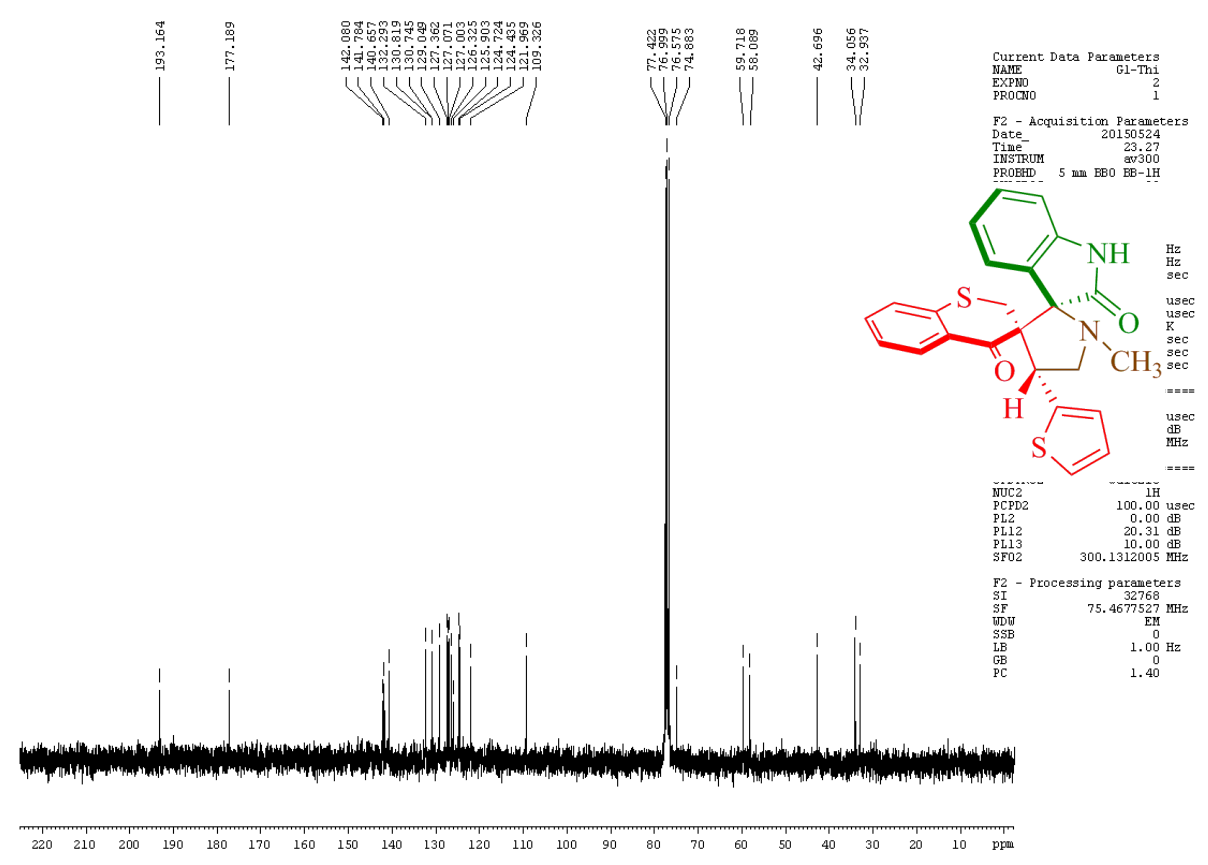

Figure. $30{ }^{13} \mathrm{C}$ NMR Spectrum of $4\{12,1,1\} \quad\left(\mathrm{CDCl}_{3}\right)$

G12NL \#22 RT: 0.33 AV: 1 NL: $2.29 E 3$

T: ITMS - c ESI Full ms [100.00-2000.00]

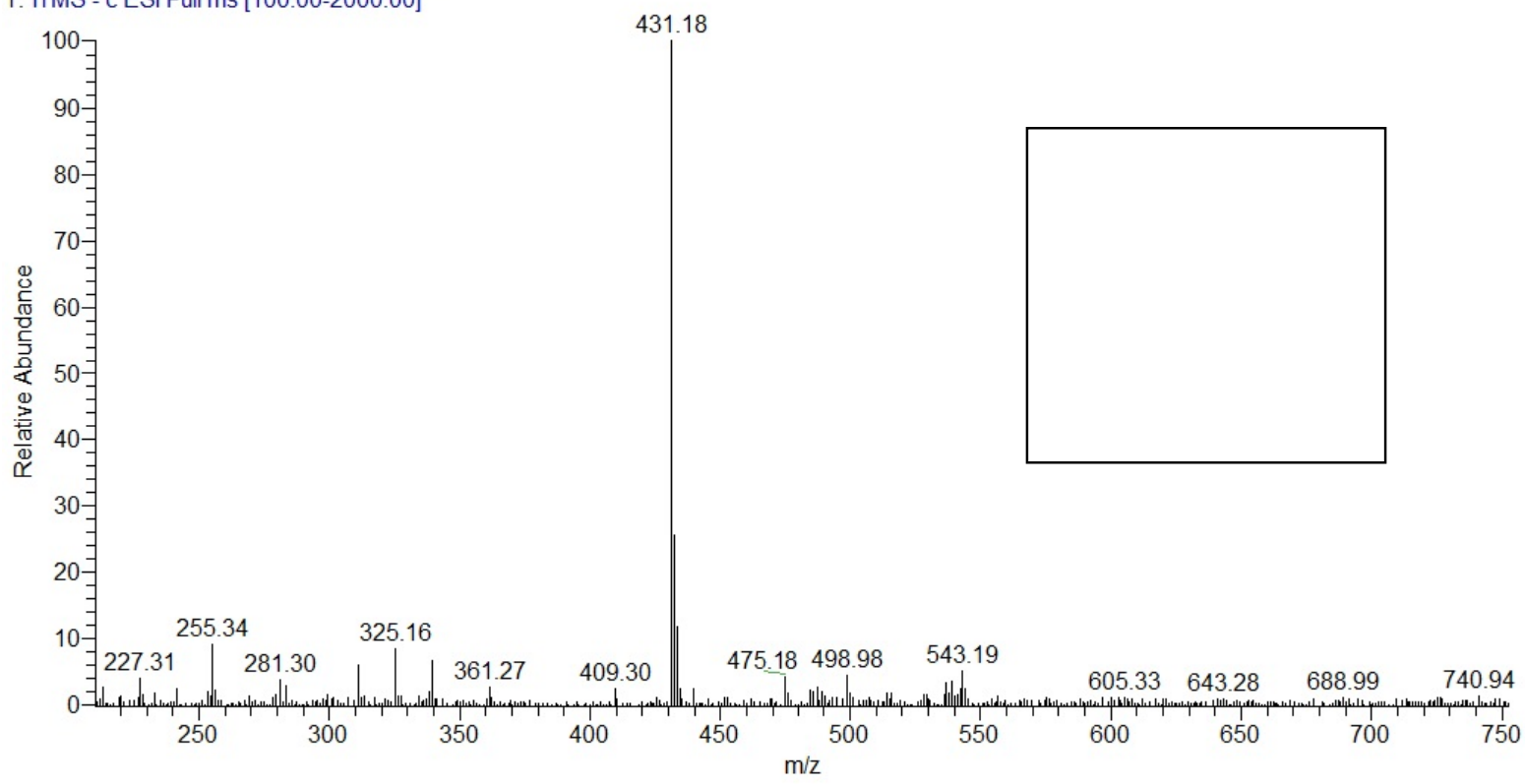

Figure. 31 ESI mass spectrum of Spectrum of $4\{12,1,1\}$ 


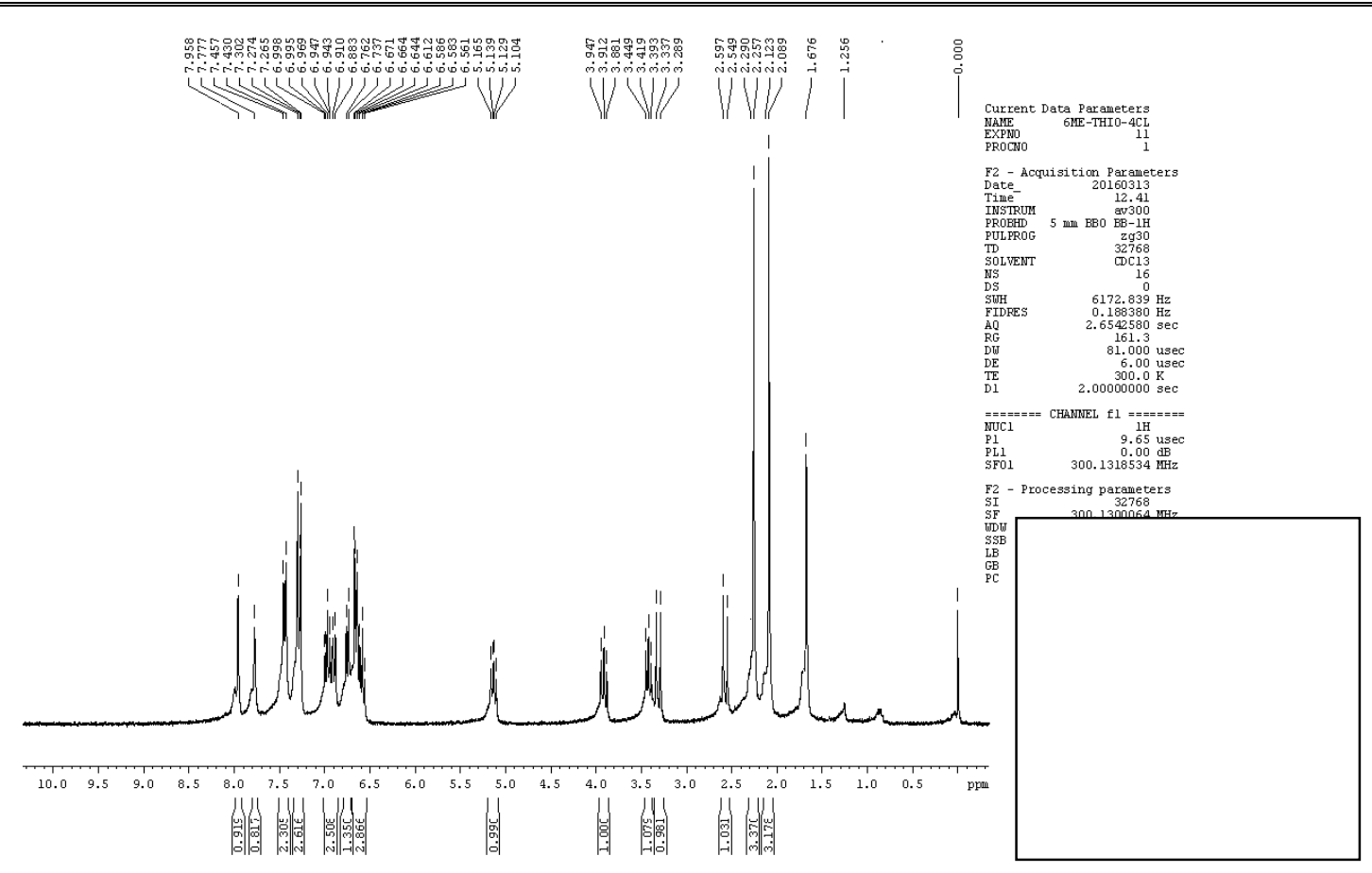

Figure. $32{ }^{1} \mathrm{H}$ NMR Spectrum $4\{13,1,1\} \quad\left(\mathrm{CDCl}_{3}\right)$

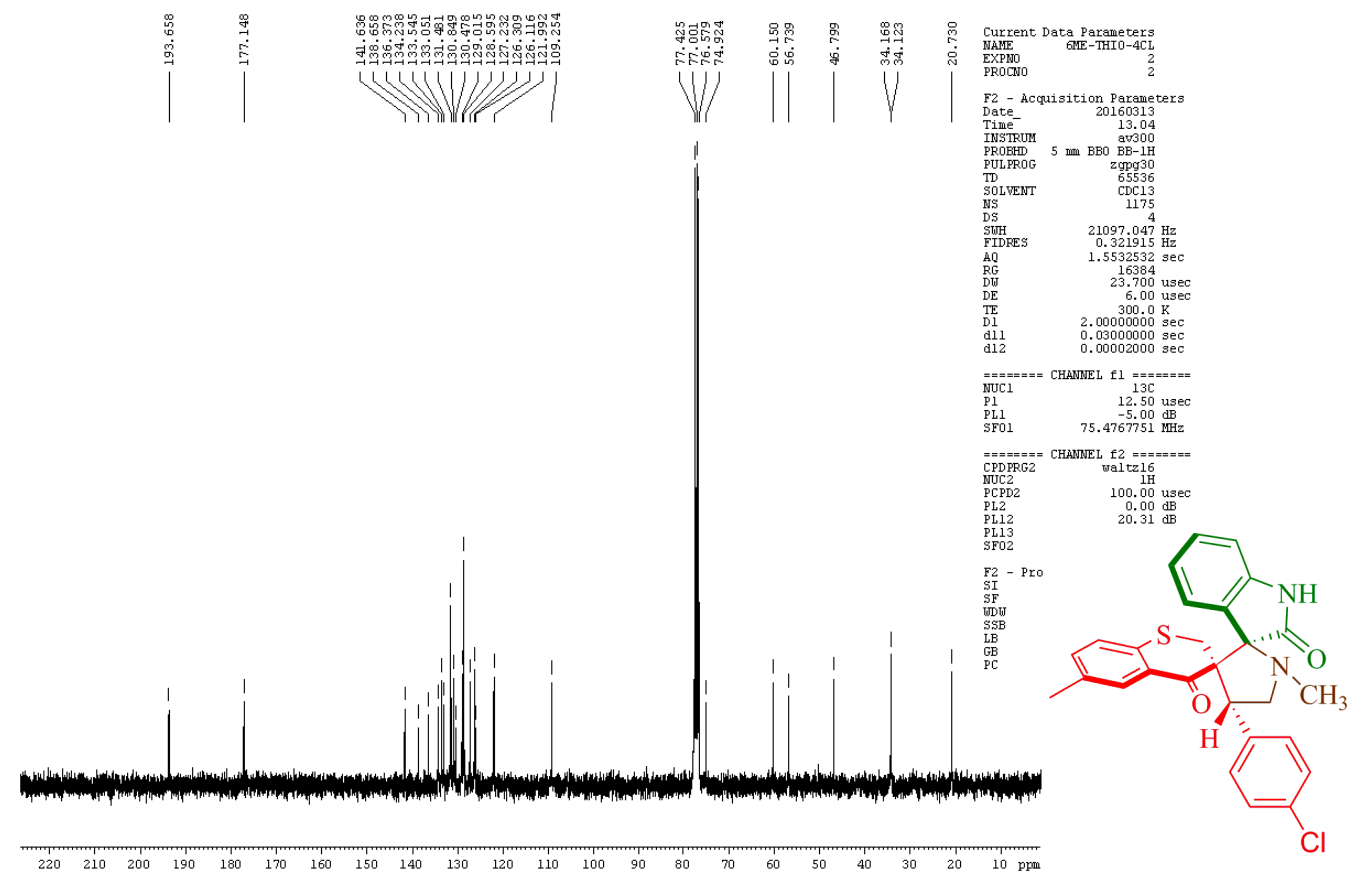

Figure. $33{ }^{13} \mathrm{C}$ NMR Spectrum of $4\{13,1,1\} \quad\left(\mathrm{CDCl}_{3}\right)$ 


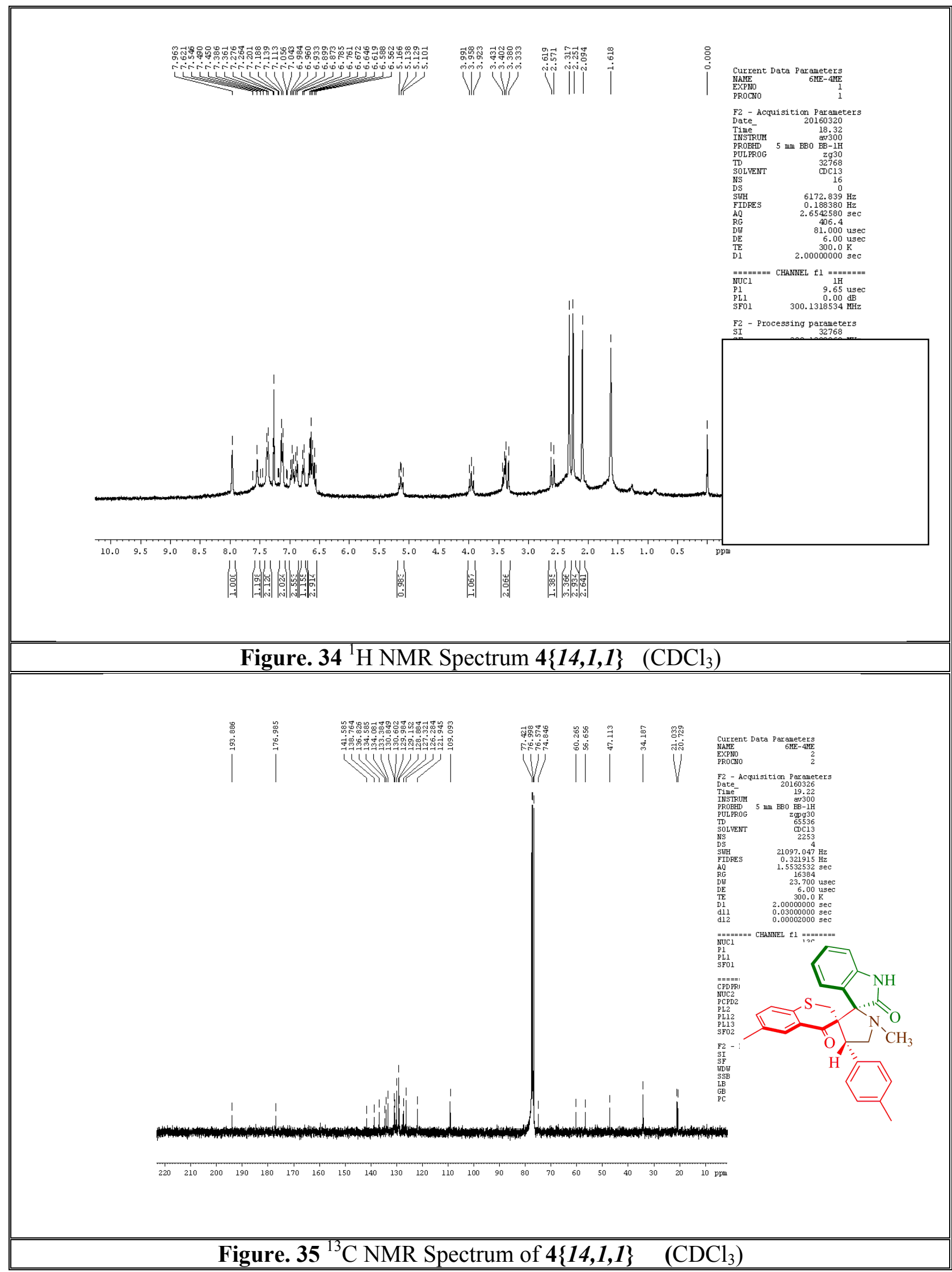




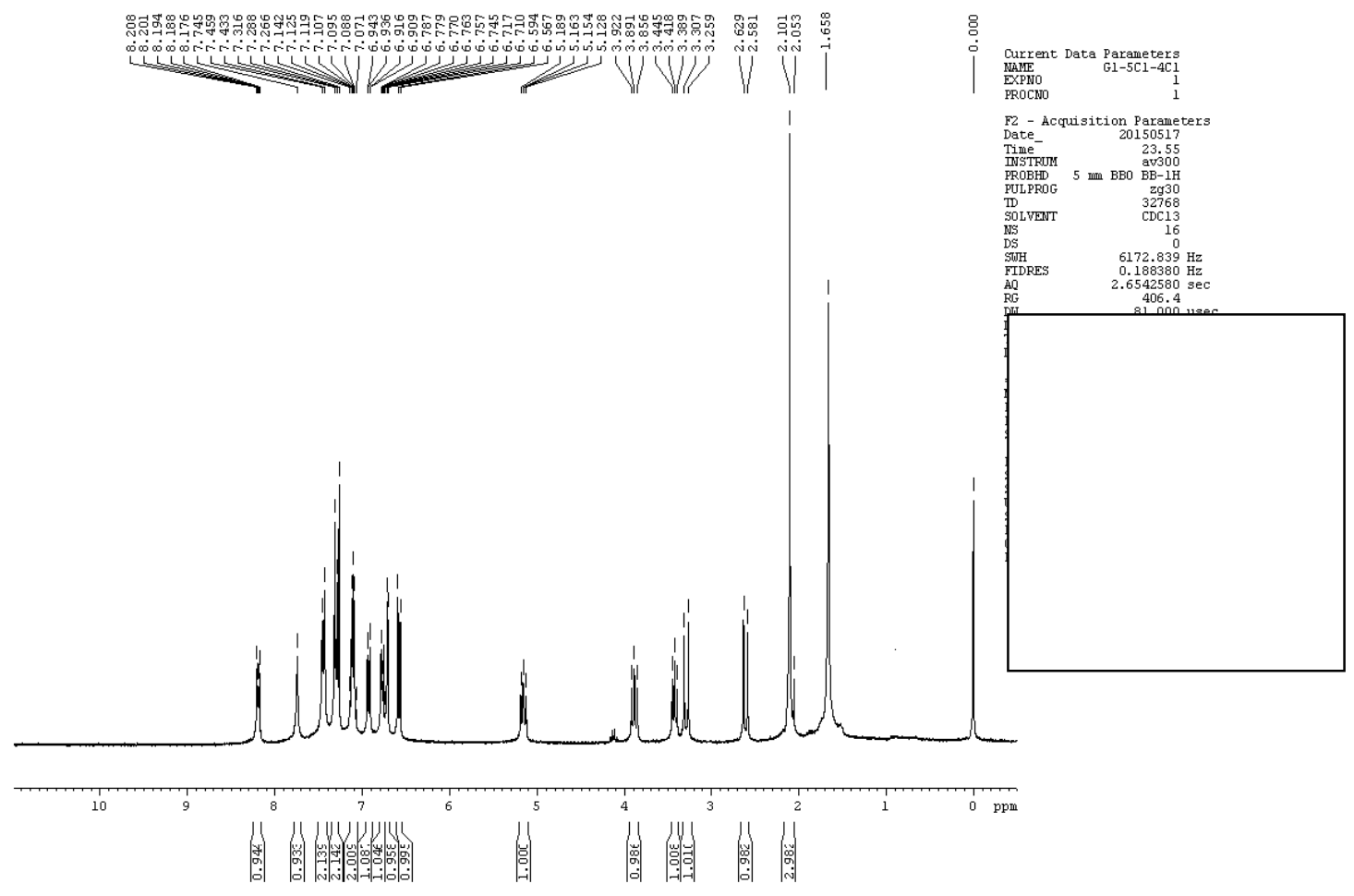

Figure. $36{ }^{1} \mathrm{H}$ NMR Spectrum 4\{2,2,1\} $\left(\mathrm{CDCl}_{3}\right)$

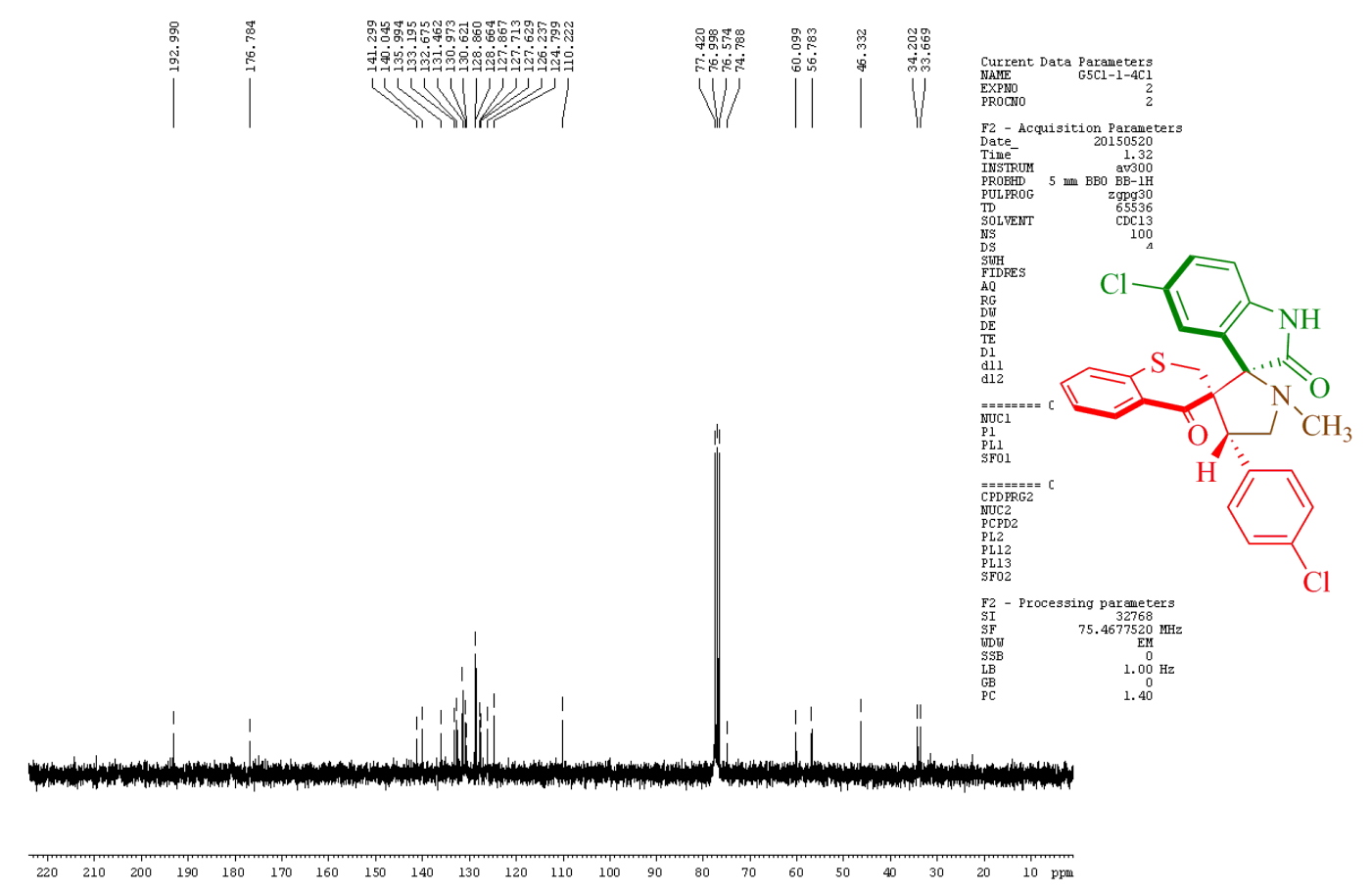

Figure. $37{ }^{13} \mathrm{C}$ NMR Spectrum of $4\{2,2,1\} \quad\left(\mathrm{CDCl}_{3}\right)$ 


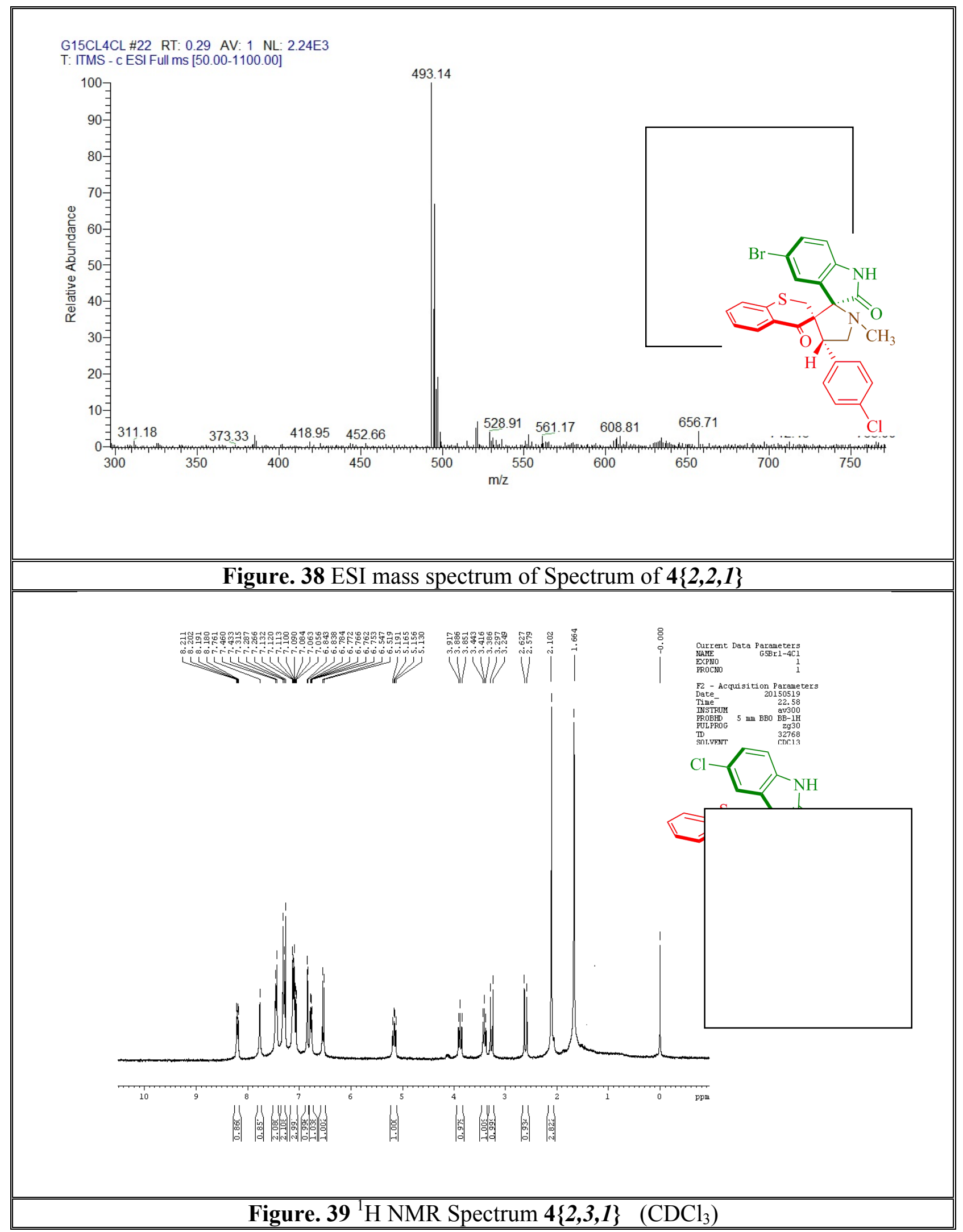




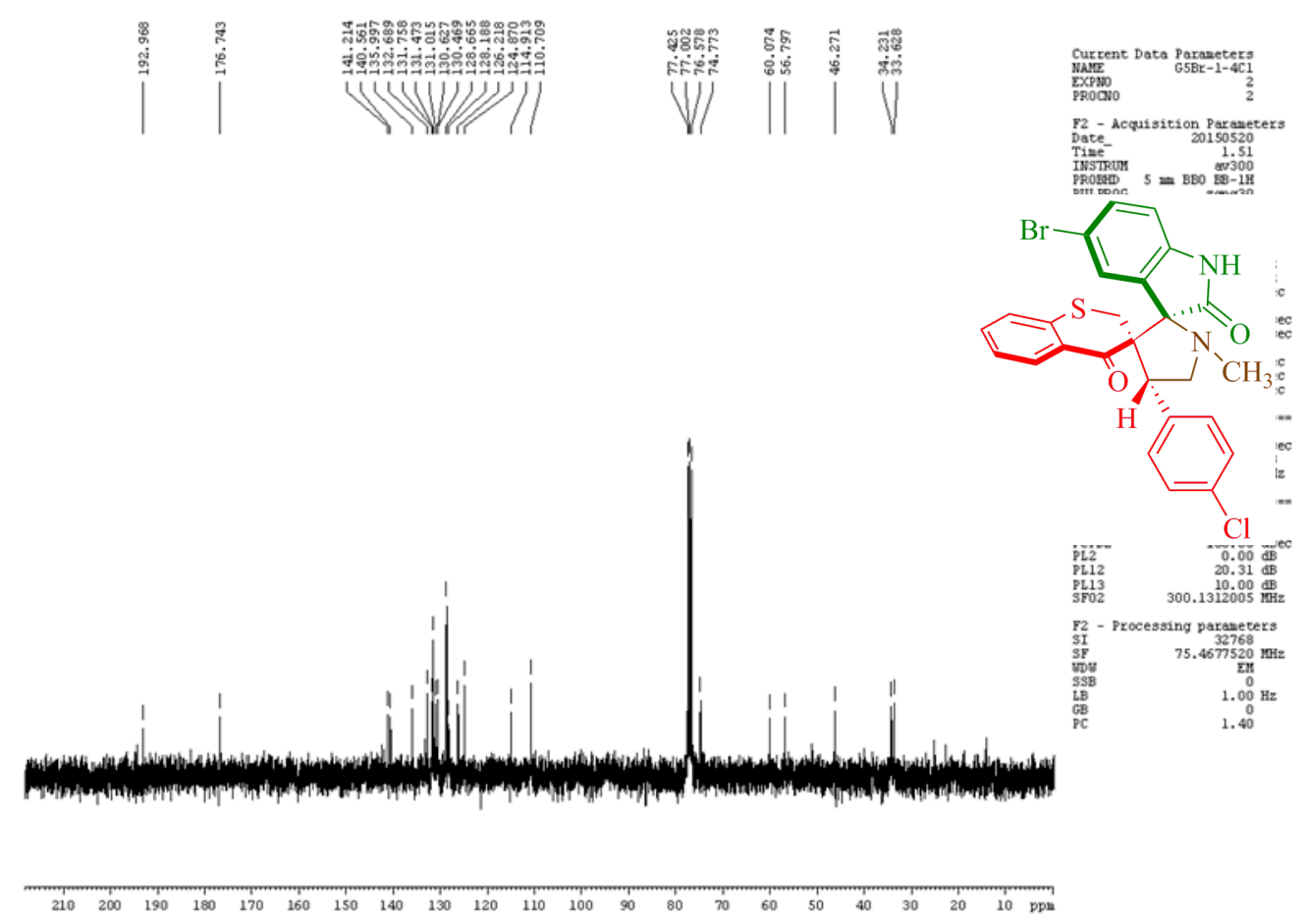

Figure. $40{ }^{13} \mathrm{C}$ NMR Spectrum of $4\{2,3,1\} \quad\left(\mathrm{CDCl}_{3}\right)$

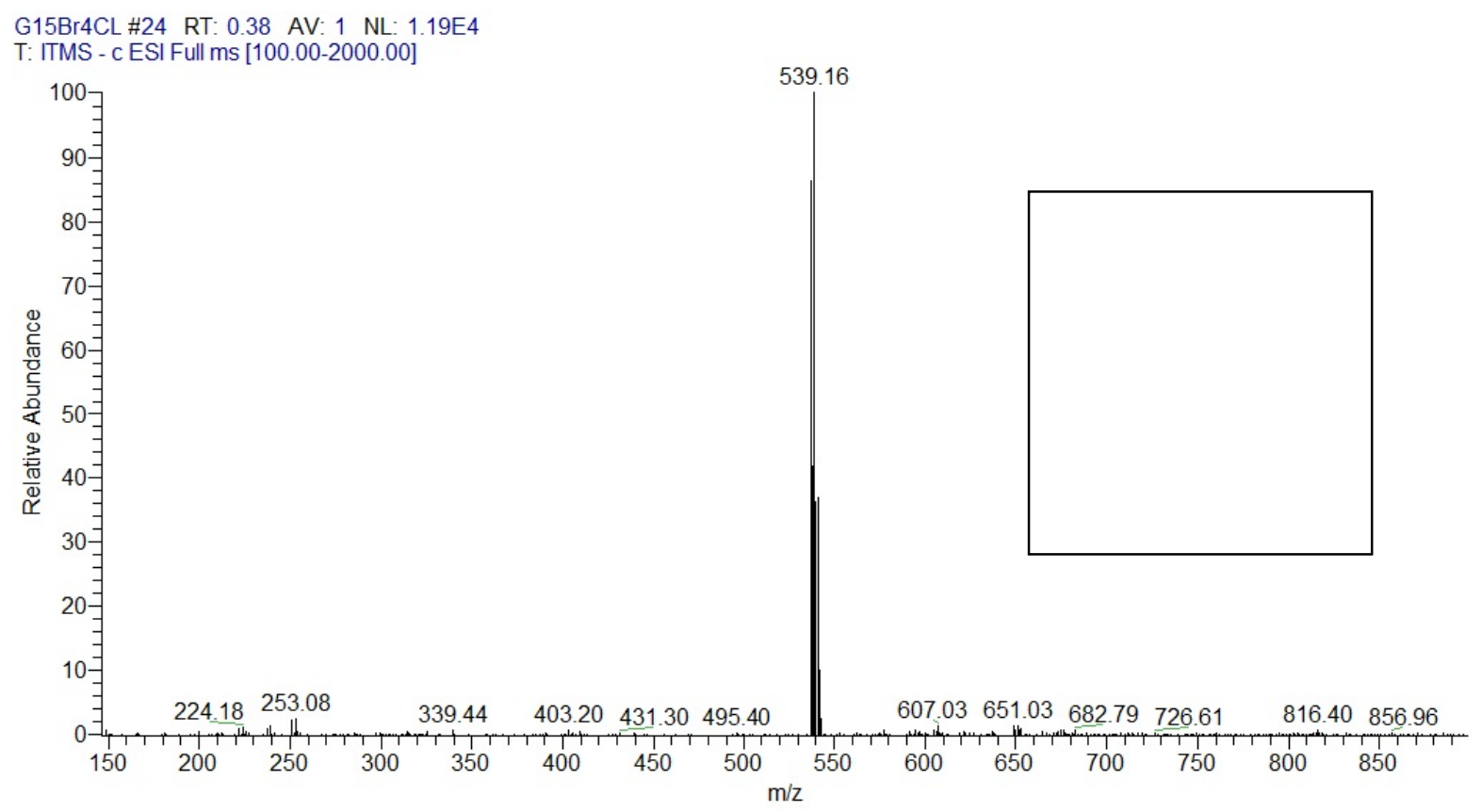

Figure. 41 ESI mass spectrum of Spectrum of $4\{2,3,1\}$ 


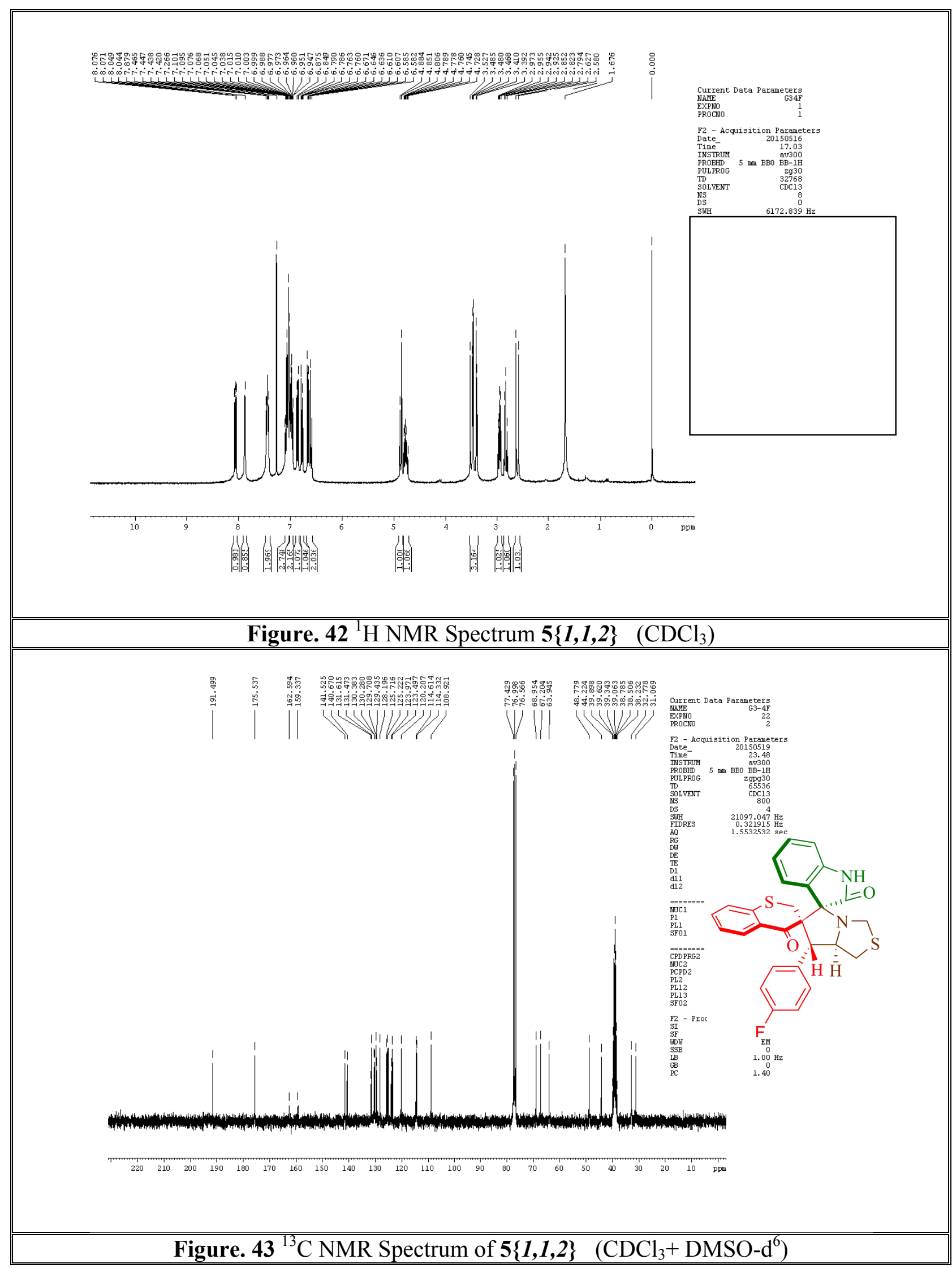




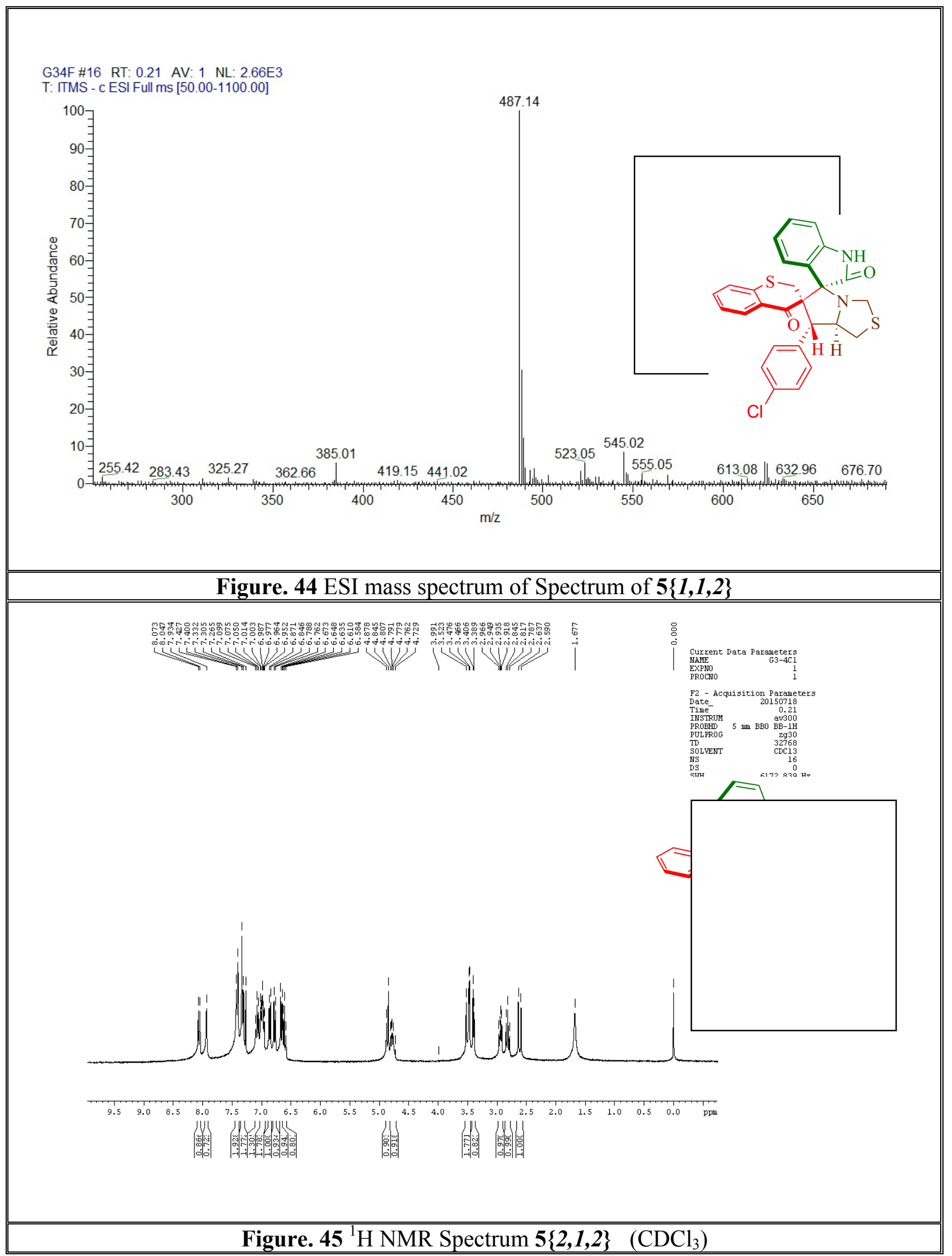




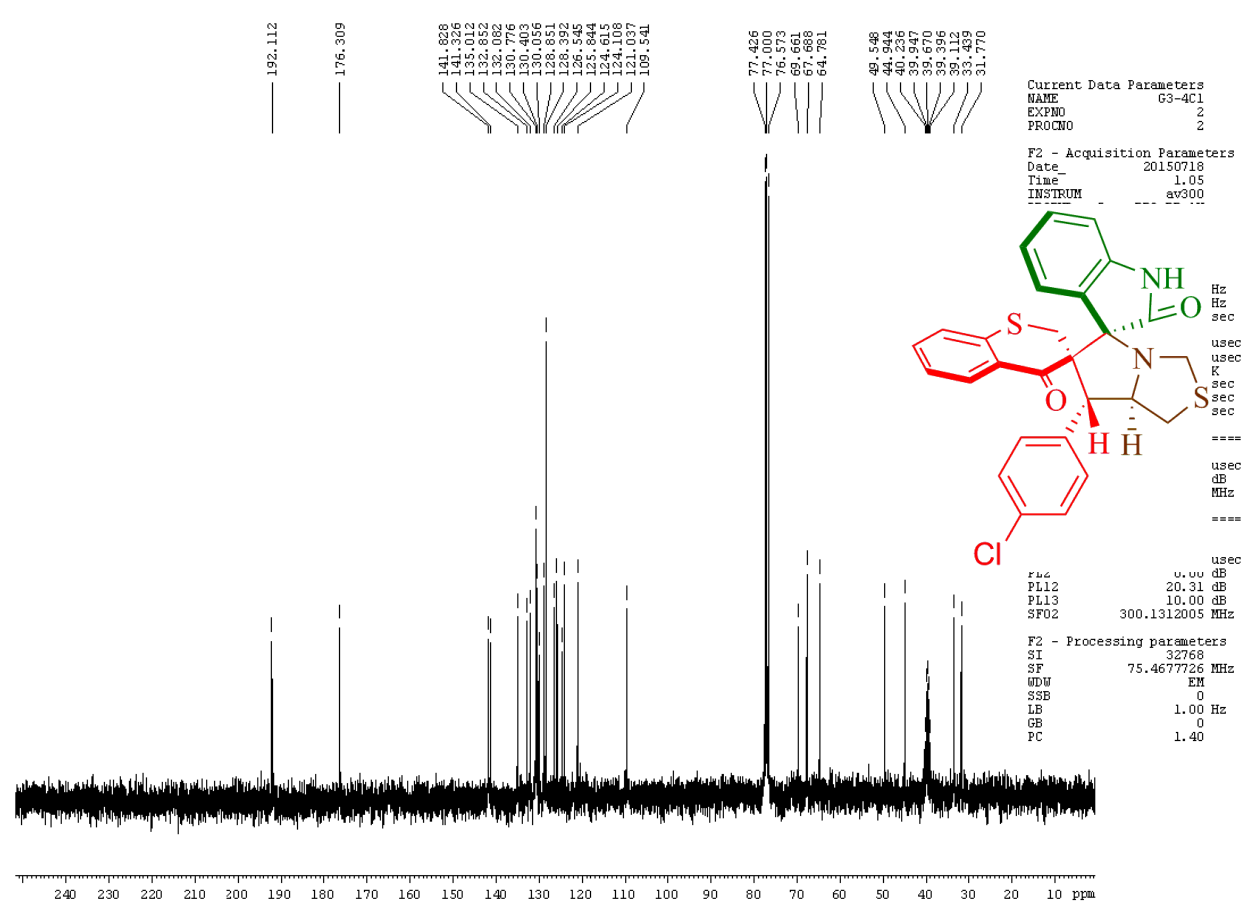

Figure. $46{ }^{13} \mathrm{C}$ NMR Spectrum of $5\{2,1,2\} \quad\left(\mathrm{CDCl}_{3}+\mathrm{DMSO}^{\mathrm{d}} \mathrm{d}^{6}\right)$

G34CL \#35 RT: 0.56 AV: 1 NL: $8.69 E 3$

T: ITMS + c ESI Full ms [100.00-1500.00]

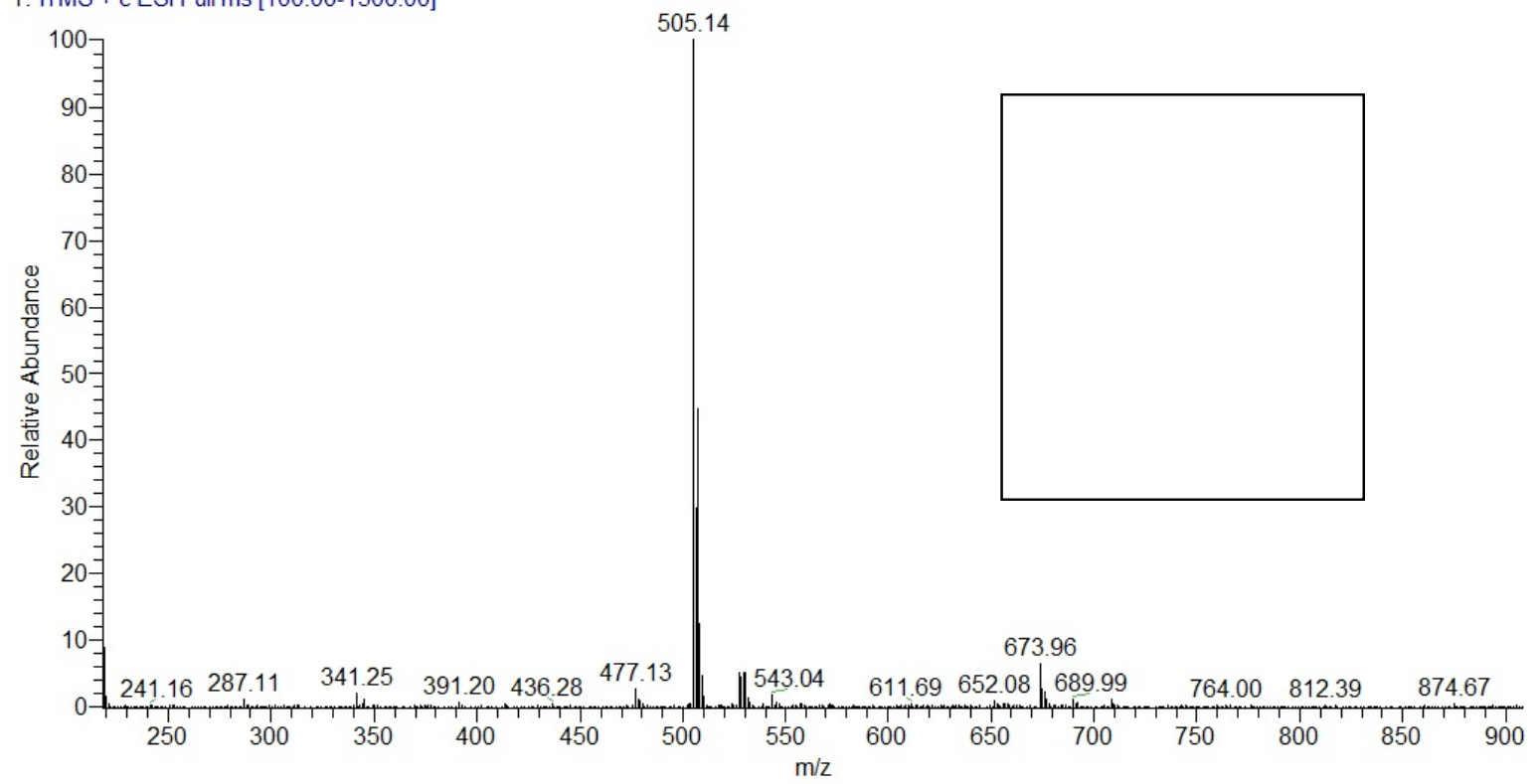

Figure. 47 ESI mass spectrum of Spectrum of $5\{2,1,2\}$ 


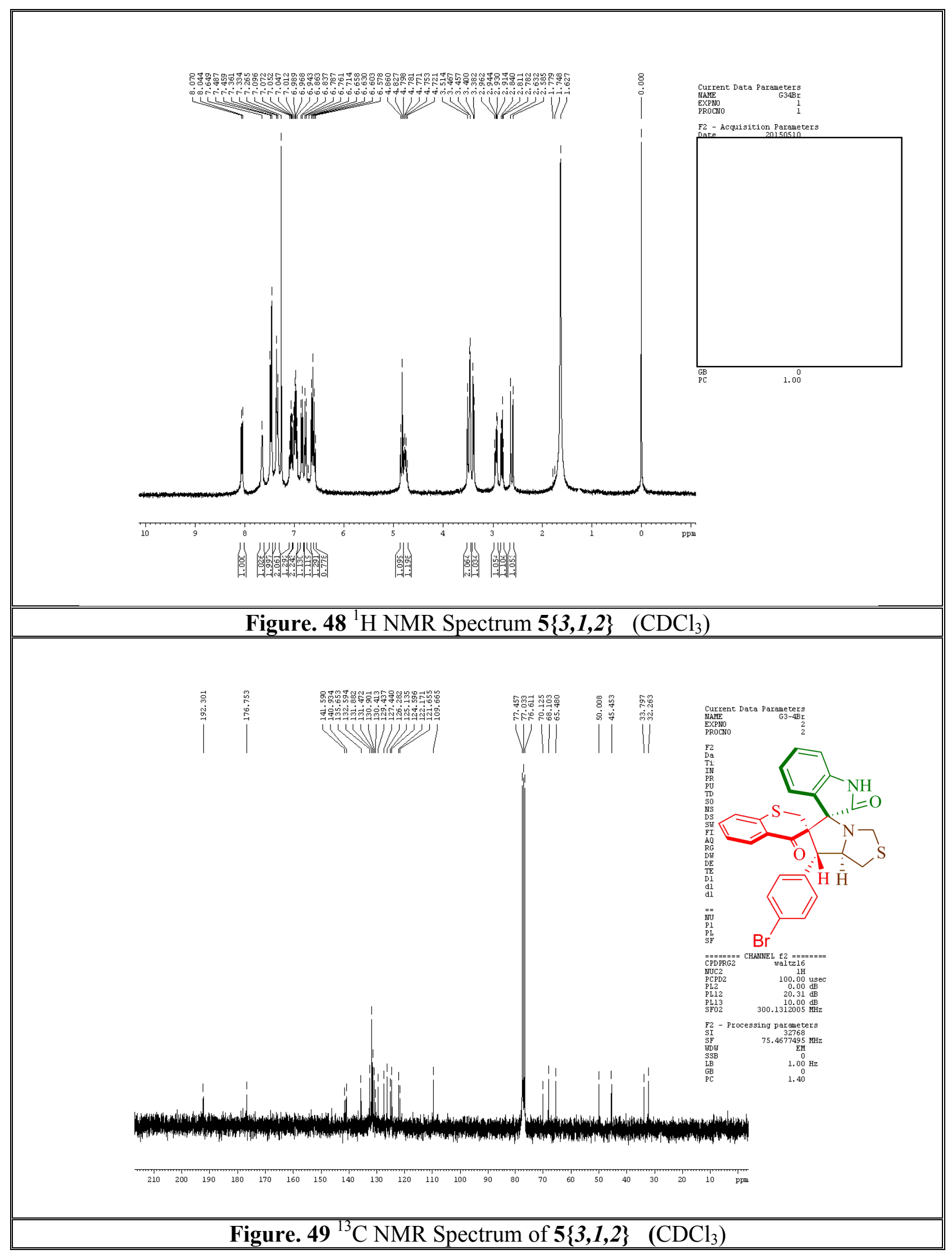


G34BR \#36 RT: 0.59 AV: 1 NL: $1.56 E 3$

T: ITMS - c ESI Full ms [100.00-1500.00]

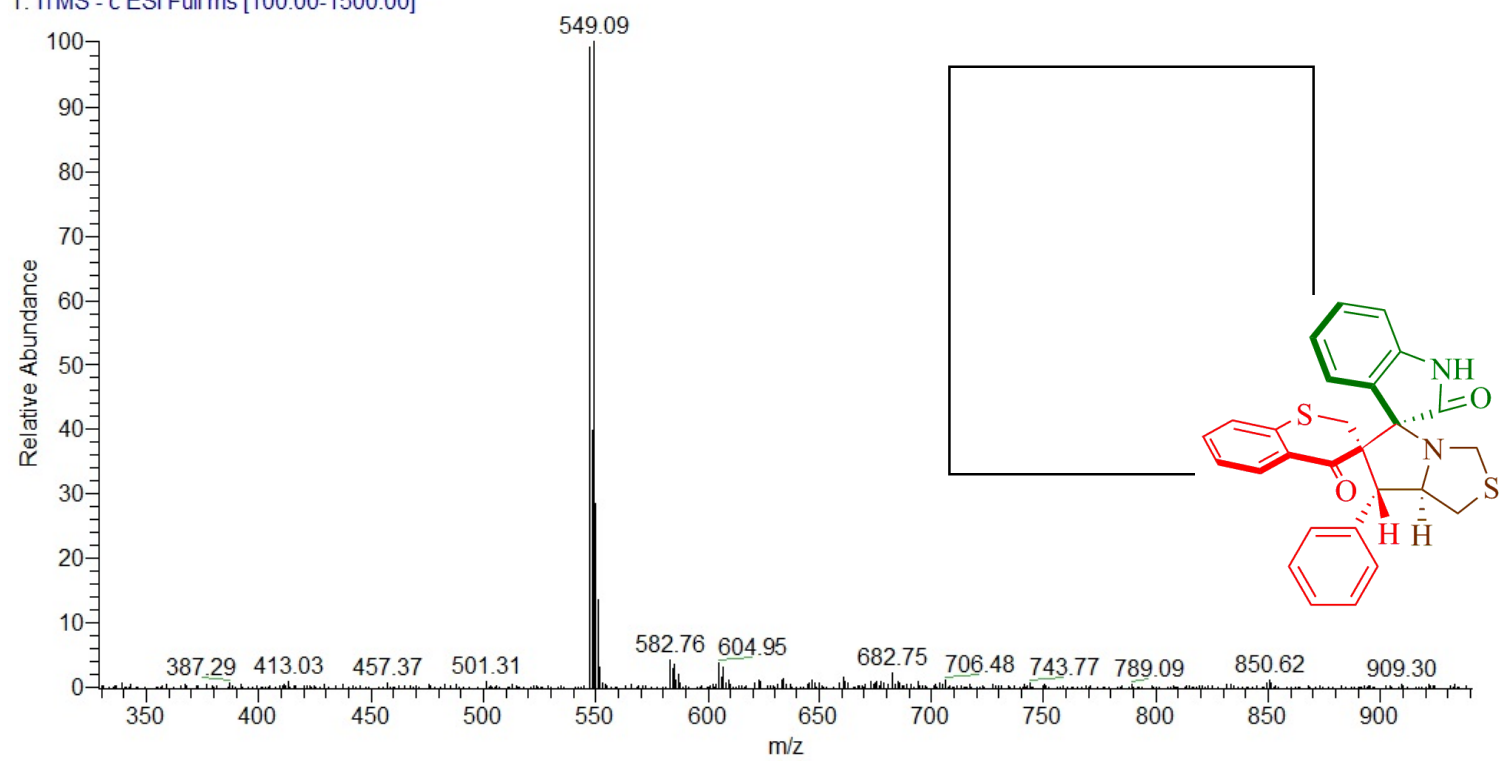

Figure. 50 ESI mass spectrum of Spectrum of $\mathbf{5}\{3,1,2\}$

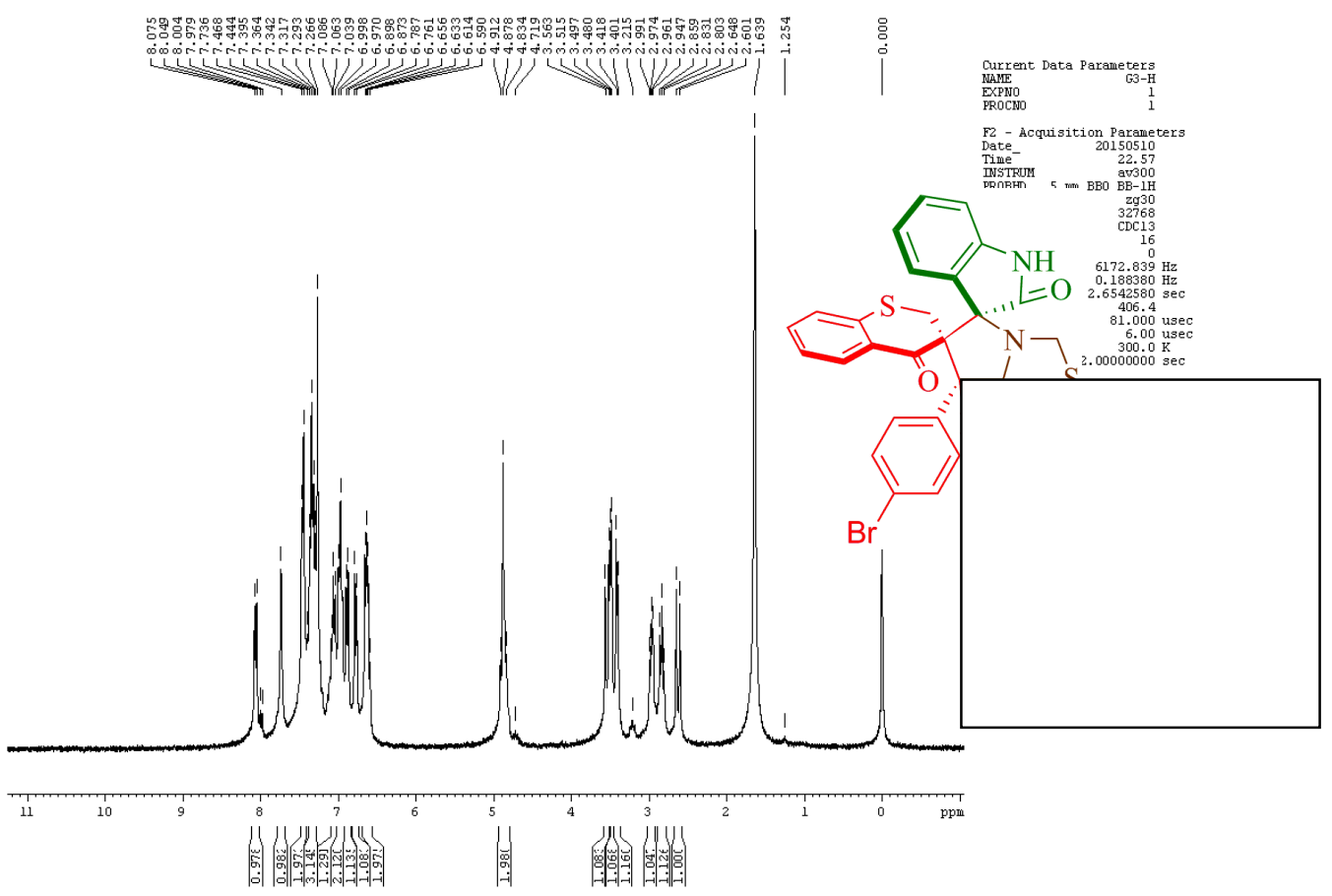

Figure. $51{ }^{1} \mathrm{H}$ NMR Spectrum 5\{4,1,2\} $\left(\mathrm{CDCl}_{3}\right)$ 


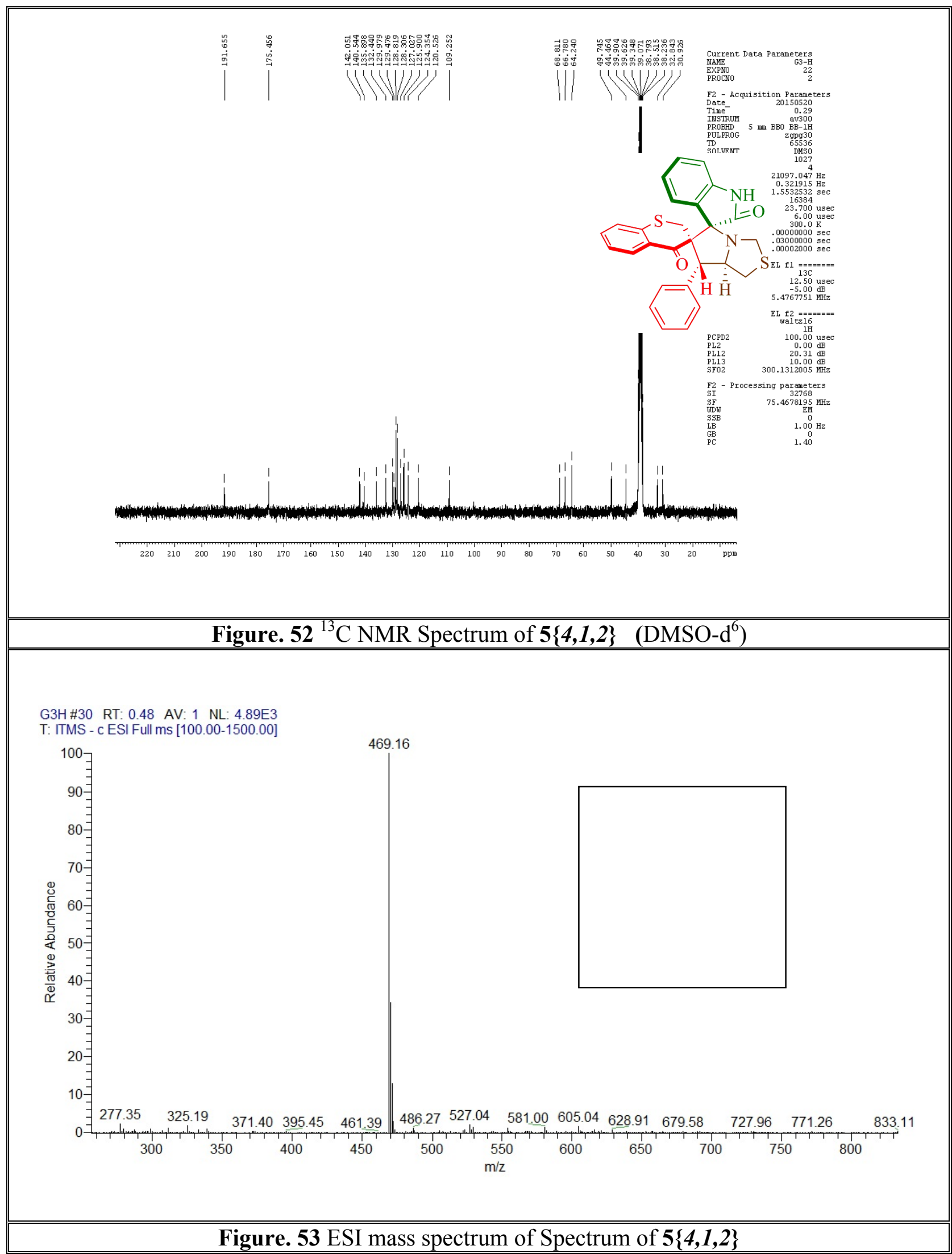




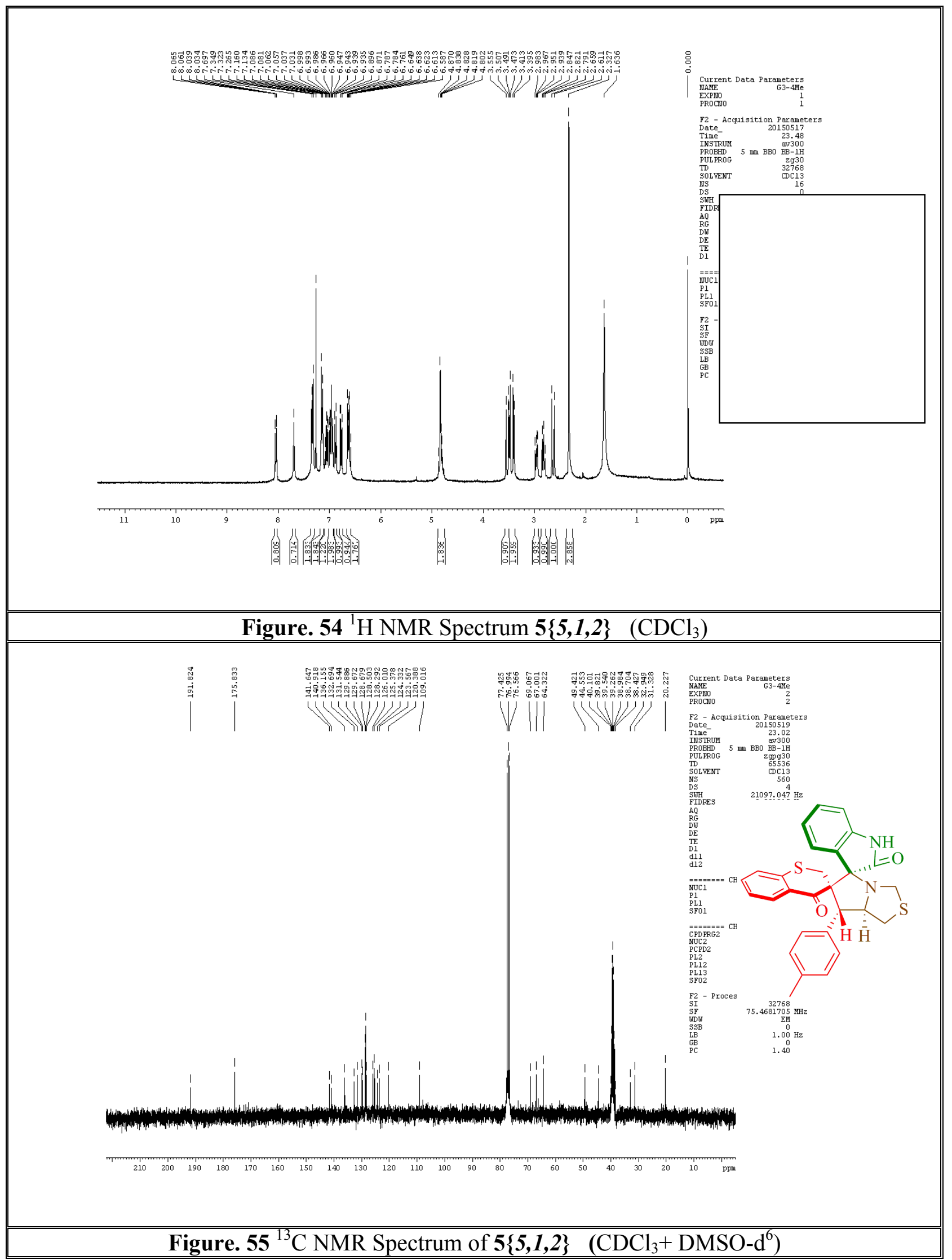


G34ME \#34 RT: 0.54 AV: 1 NL: 6.74E3

T: ITMS - c ESI Full ms [100.00-1500.00]

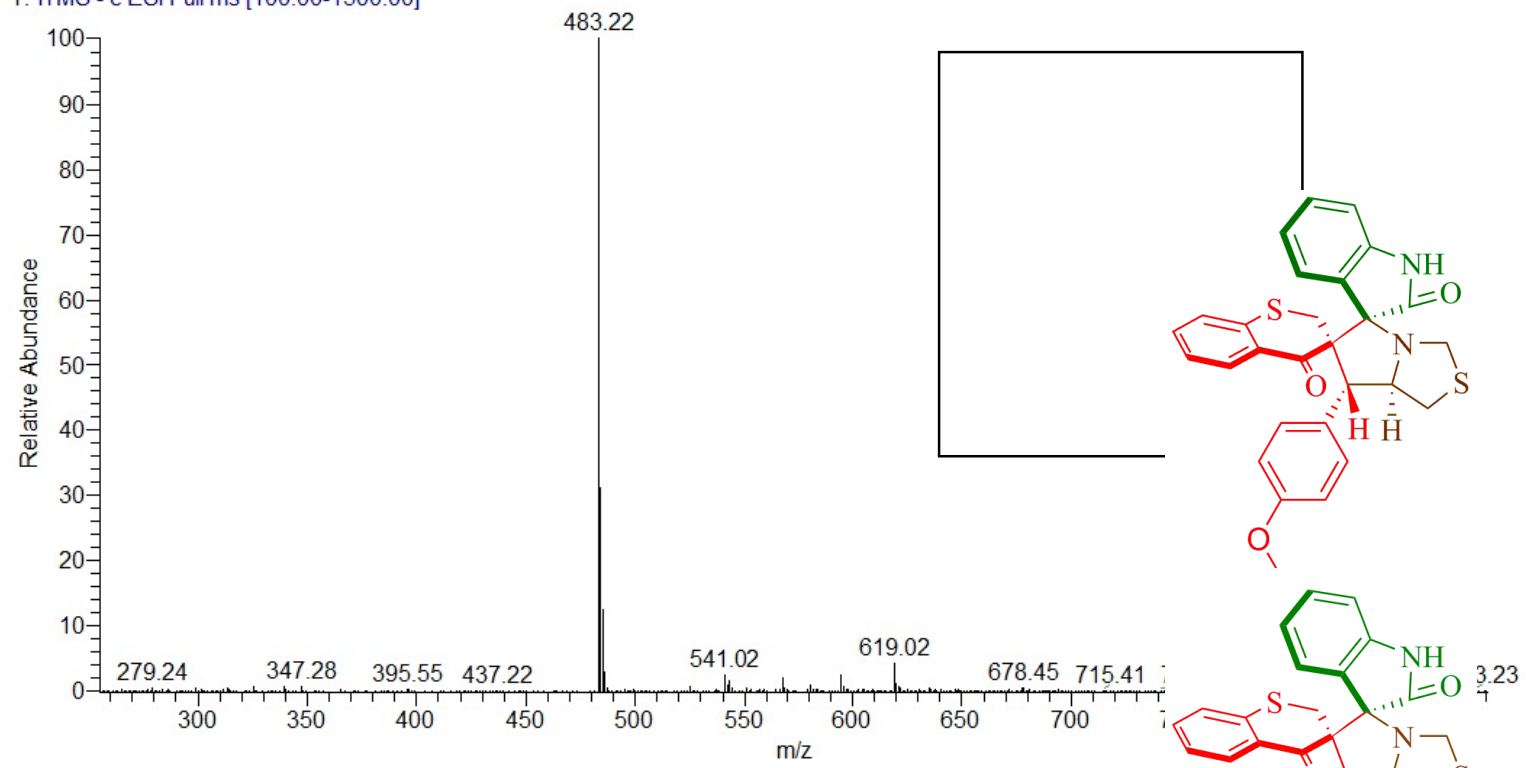

Figure. 56 ESI mass spectrum of Spectrum of $5\{5,1,2\}$

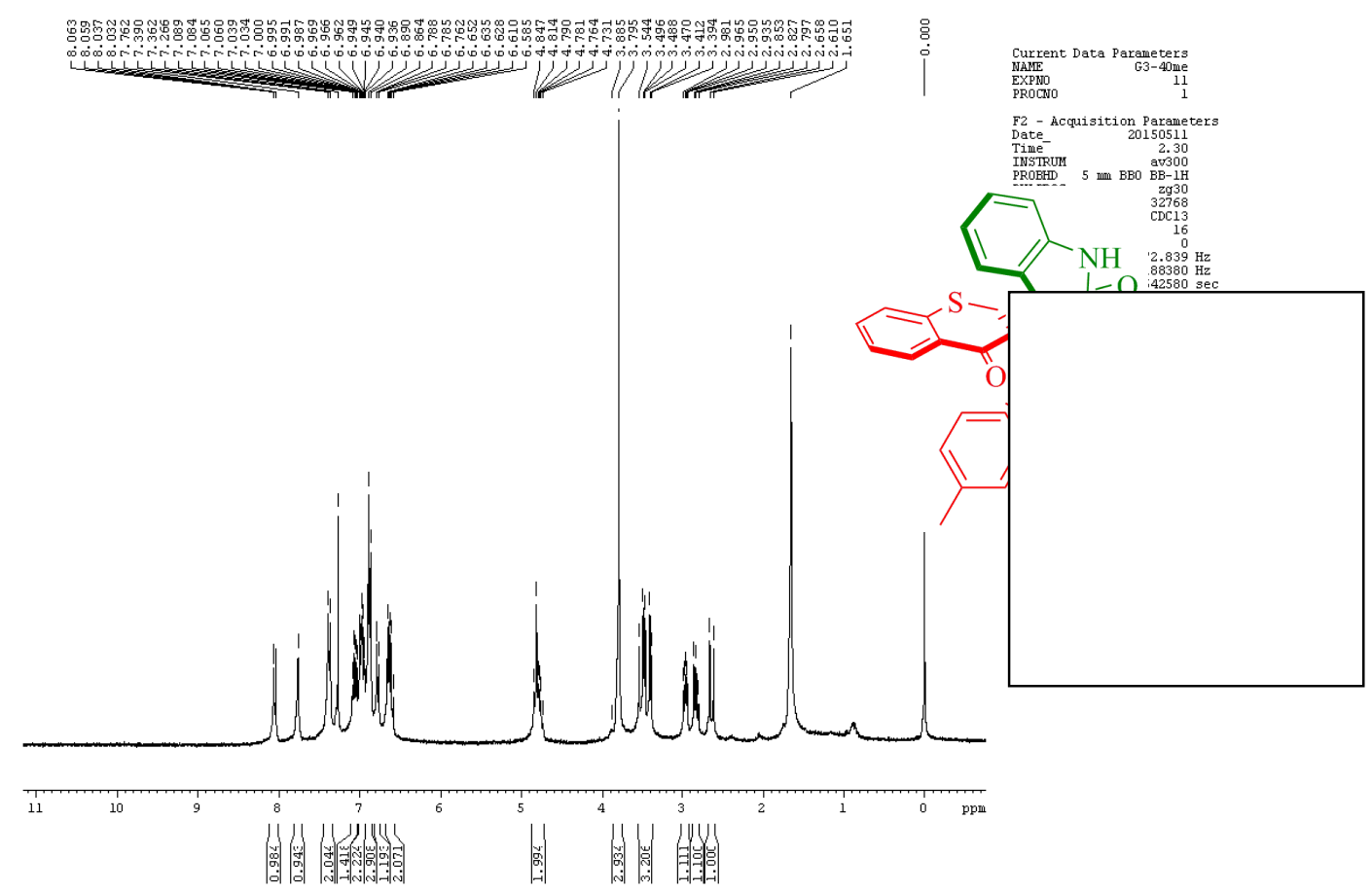

Figure. $57^{1} \mathrm{H}$ NMR Spectrum 5\{6,1,2\} $\left(\mathrm{CDCl}_{3}\right)$ 


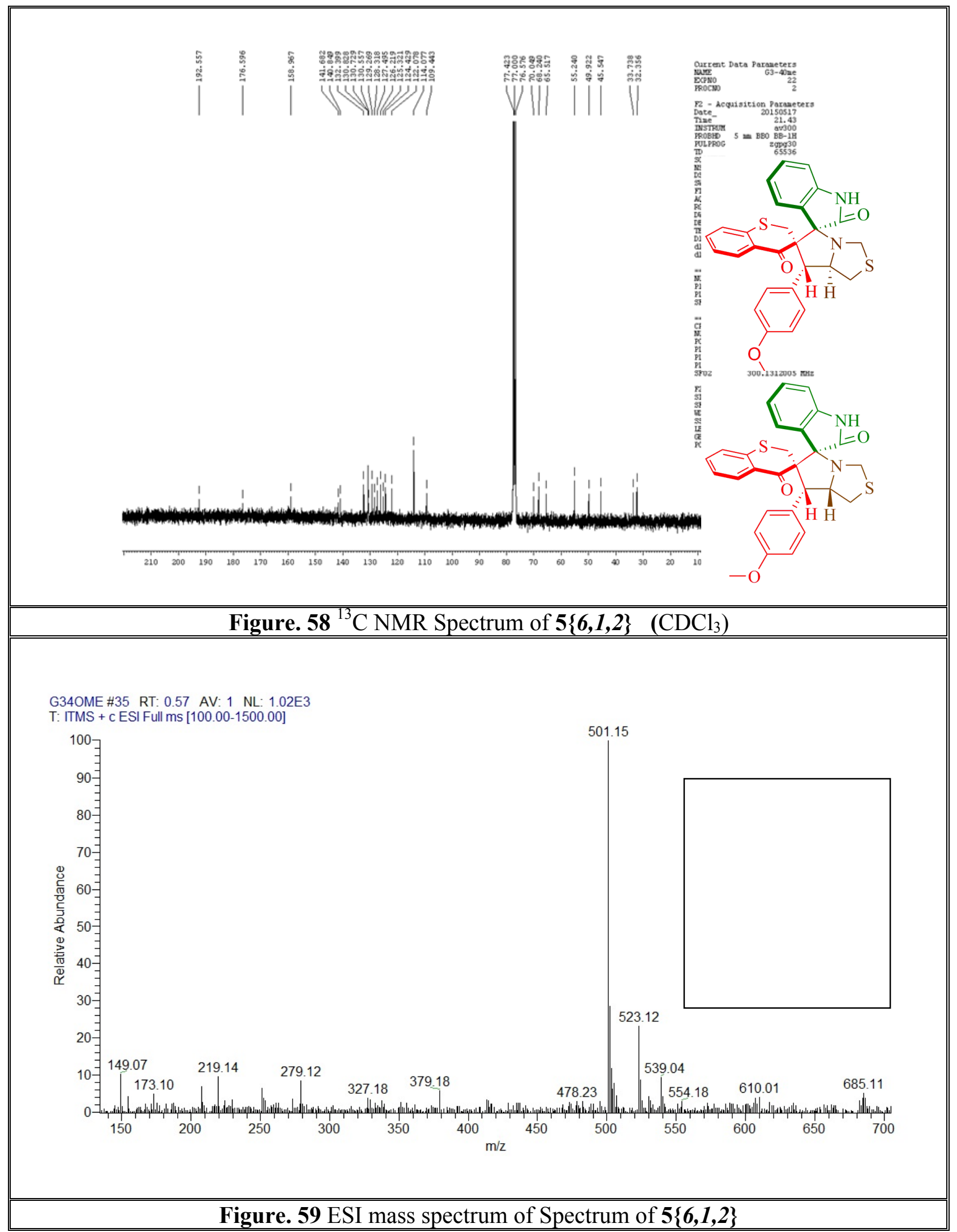




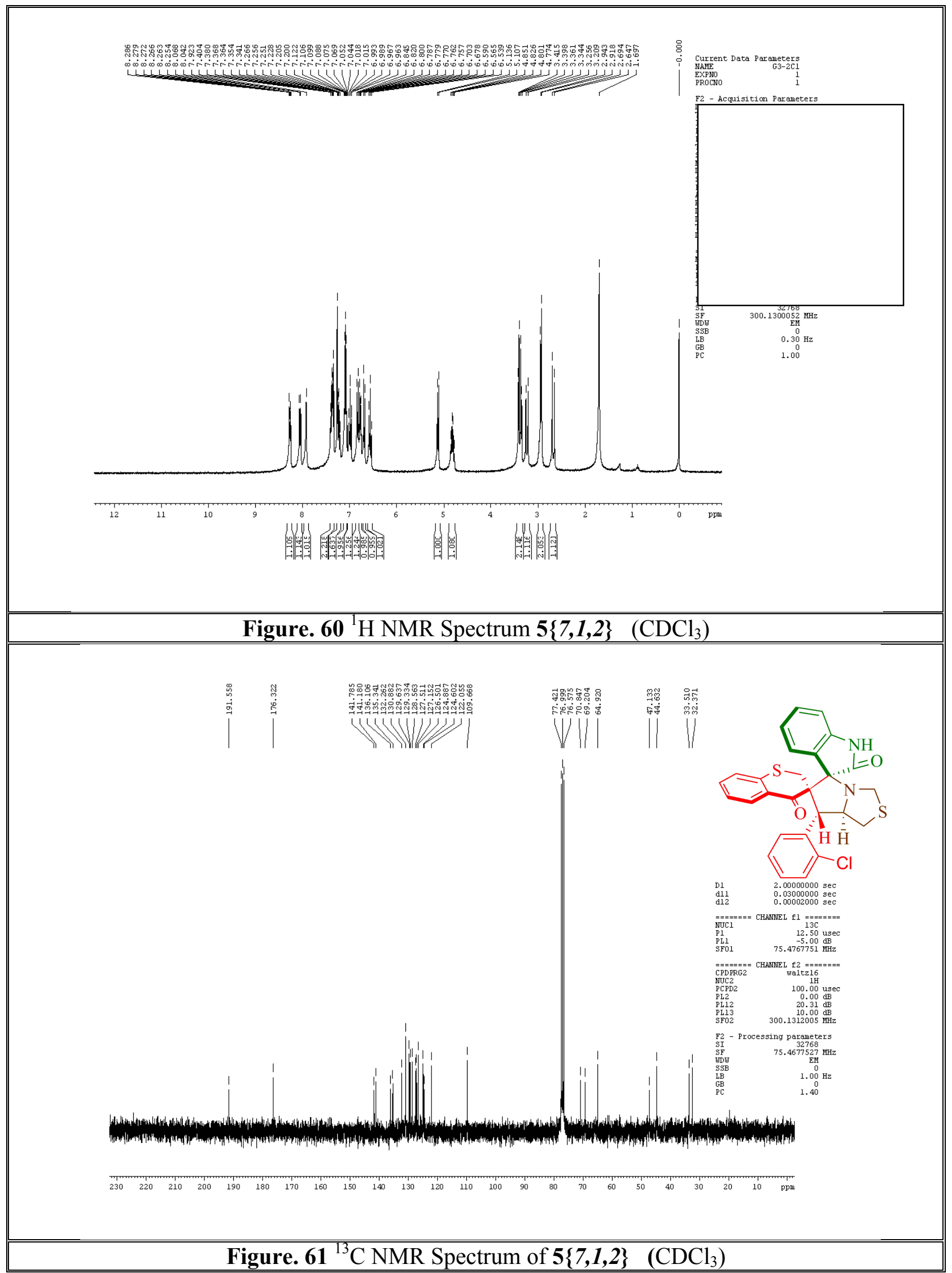


$332 \mathrm{CL} \# 32$ RT: 0.51 AV: 1 NL: $2.87 \mathrm{E} 3$

Г: ITMS - c ESI Full ms [100.00-1500.00]
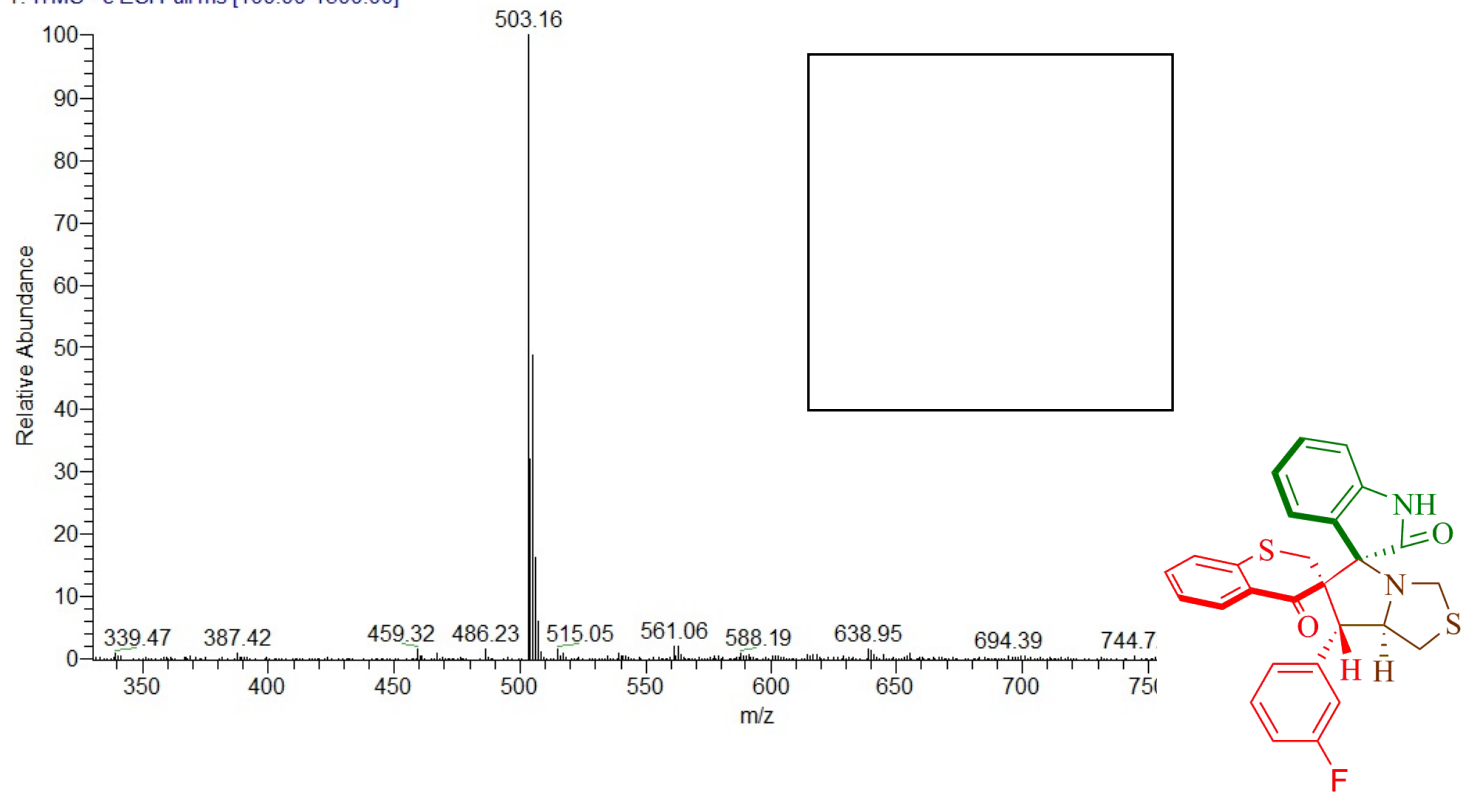

Figure. 62 ESI mass spectrum of Spectrum of $\mathbf{5}\{\mathbf{7 , 1 , 2}\}$

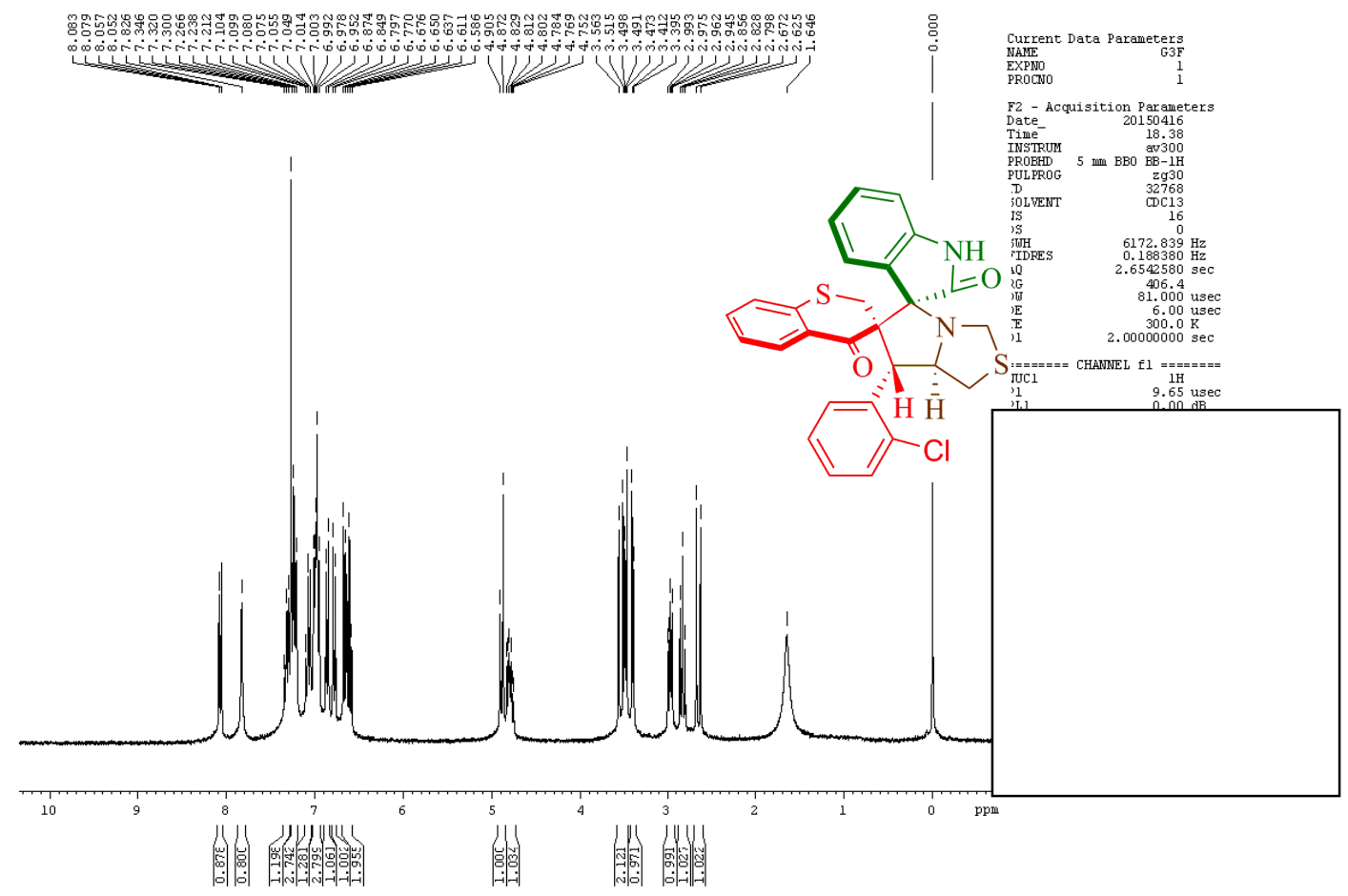

Figure. 63 ${ }^{1} \mathrm{H}$ NMR Spectrum $5\{8,1,2\} \quad\left(\mathrm{CDCl}_{3}\right)$ 


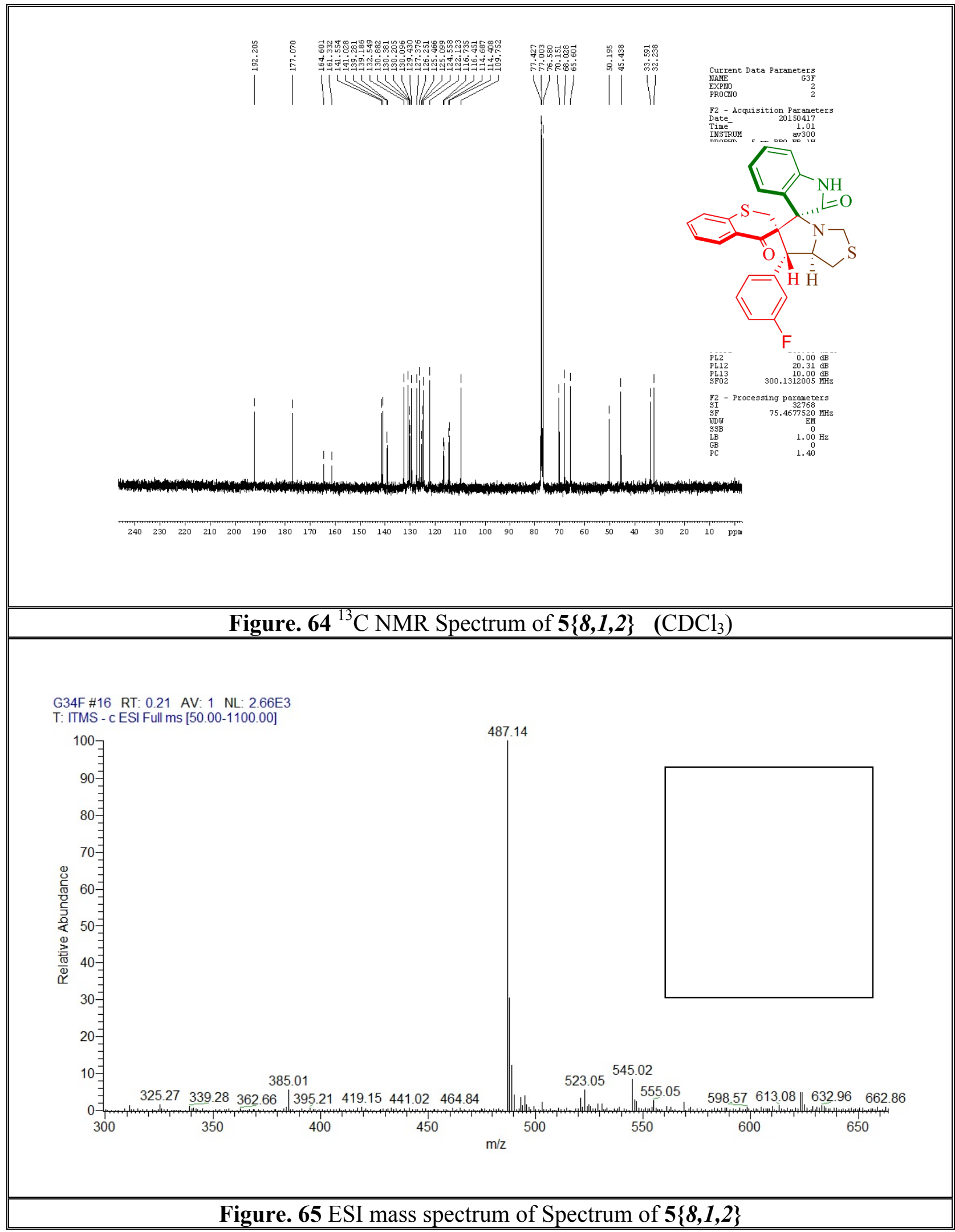




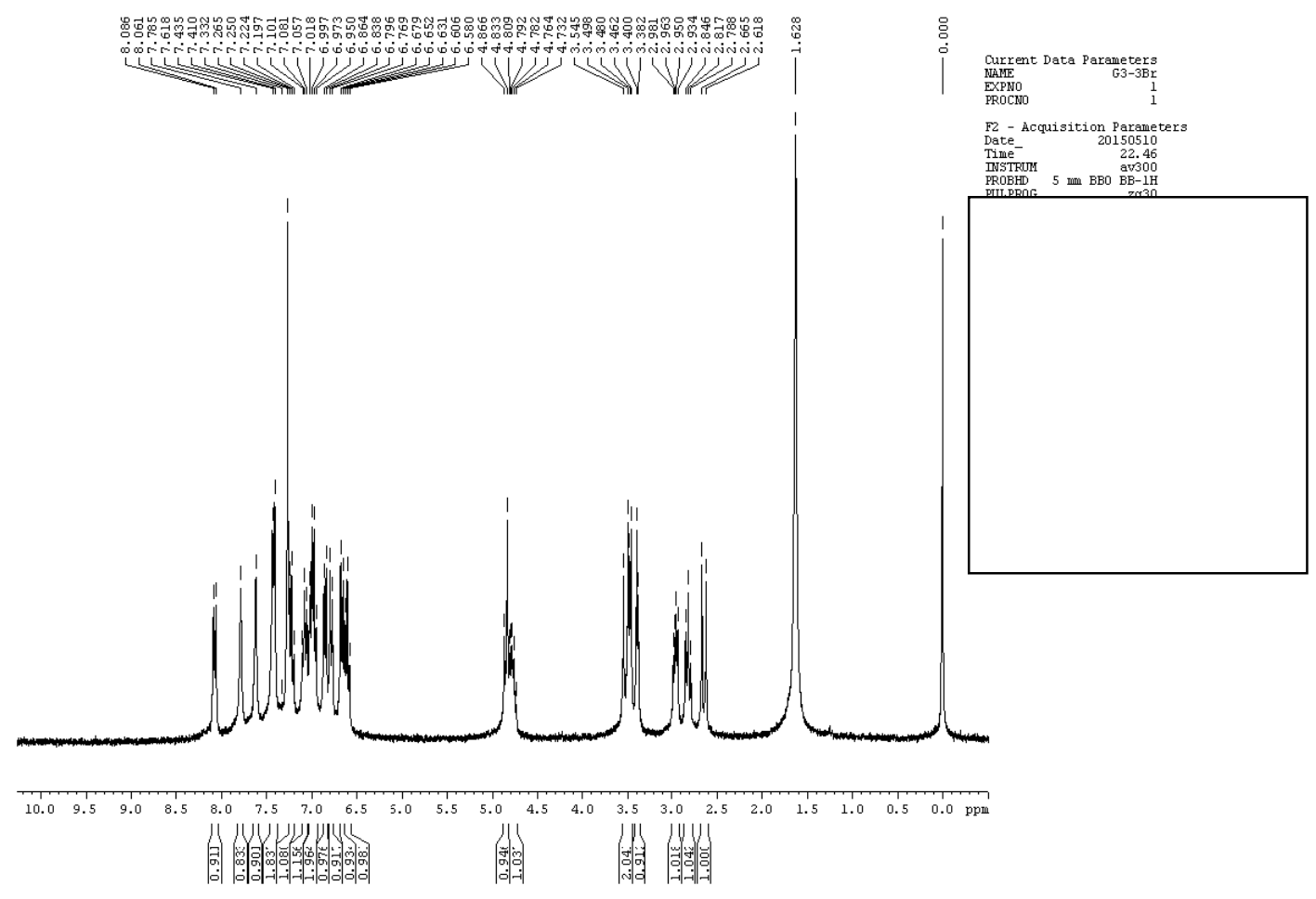

Figure. $66{ }^{1} \mathrm{H}$ NMR Spectrum 5\{9,1,2\} $\left(\mathrm{CDCl}_{3}\right)$

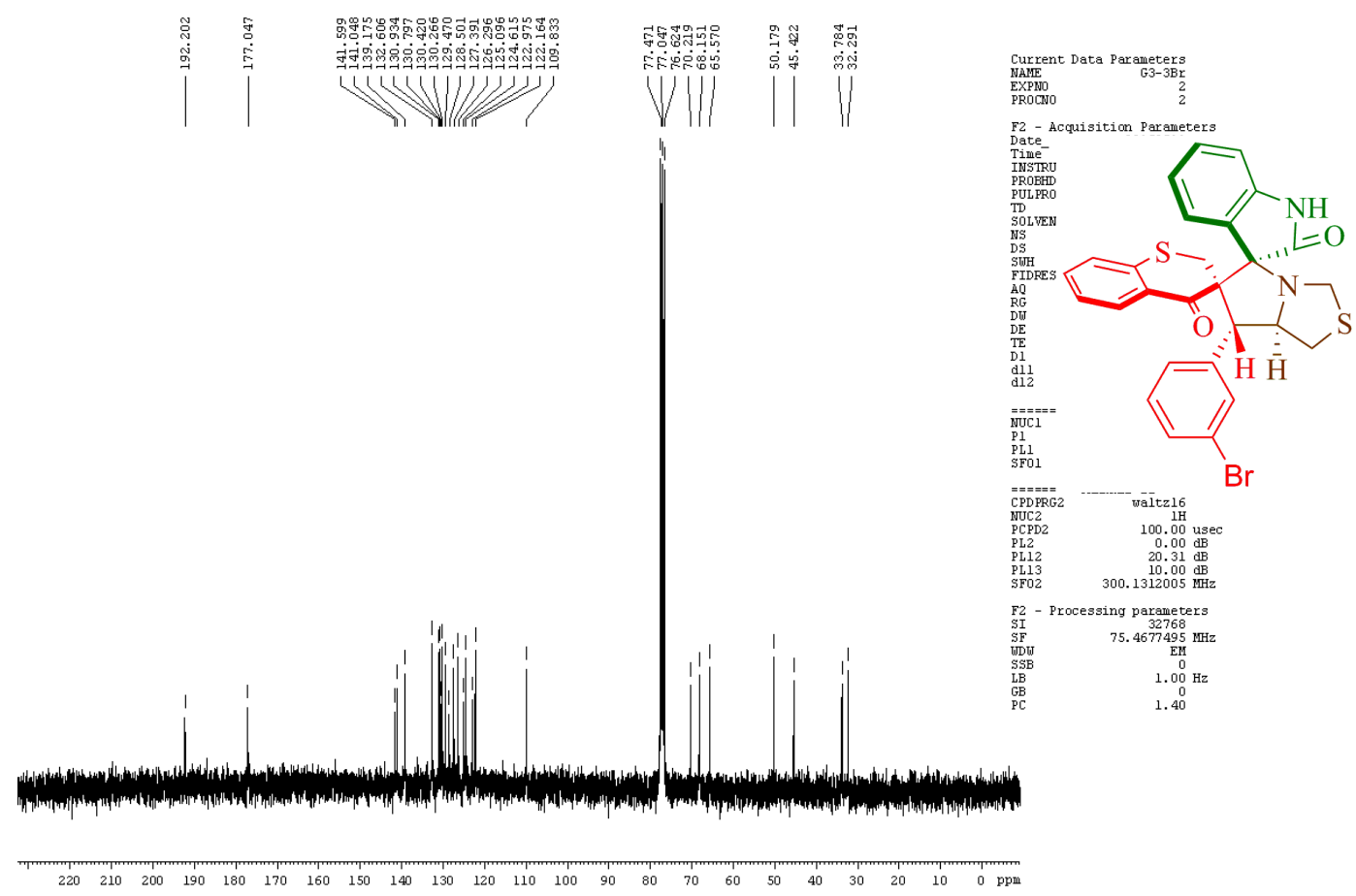

Figure. $67{ }^{13} \mathrm{C}$ NMR Spectrum of $\mathbf{5}\left\{\mathbf{9 , 1 , 2 \}}\left(\mathrm{CDCl}_{3}\right)\right.$ 


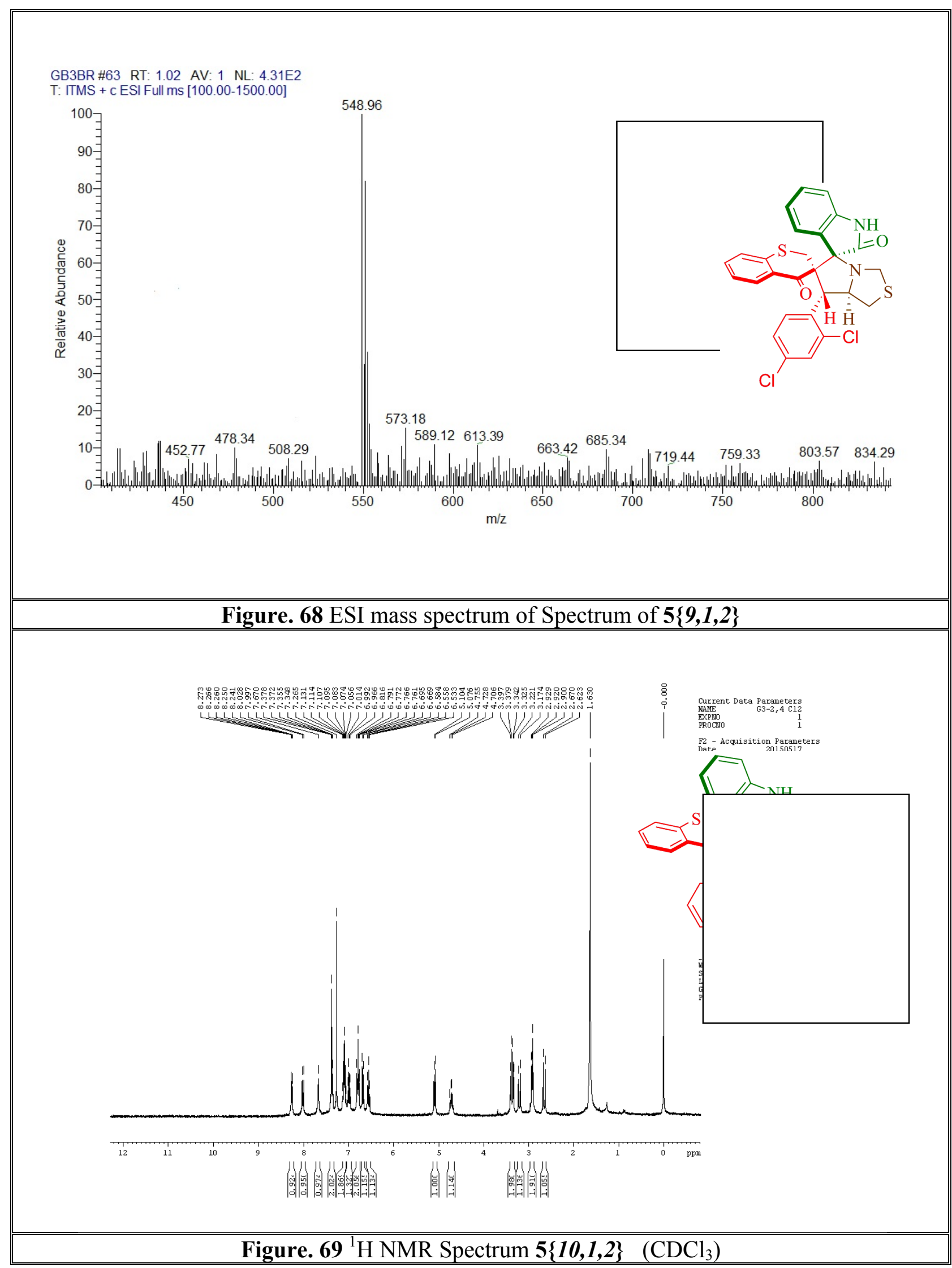




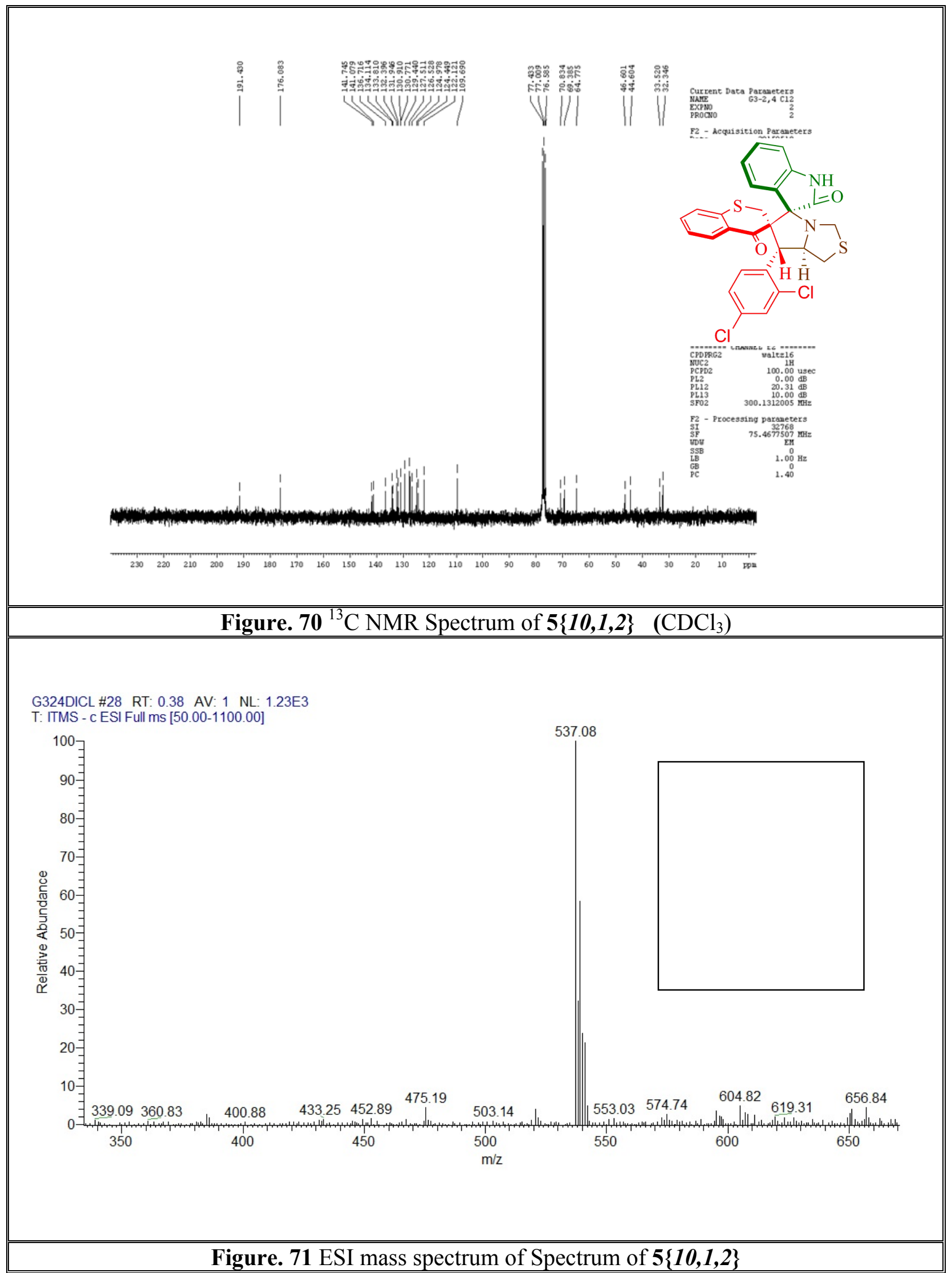




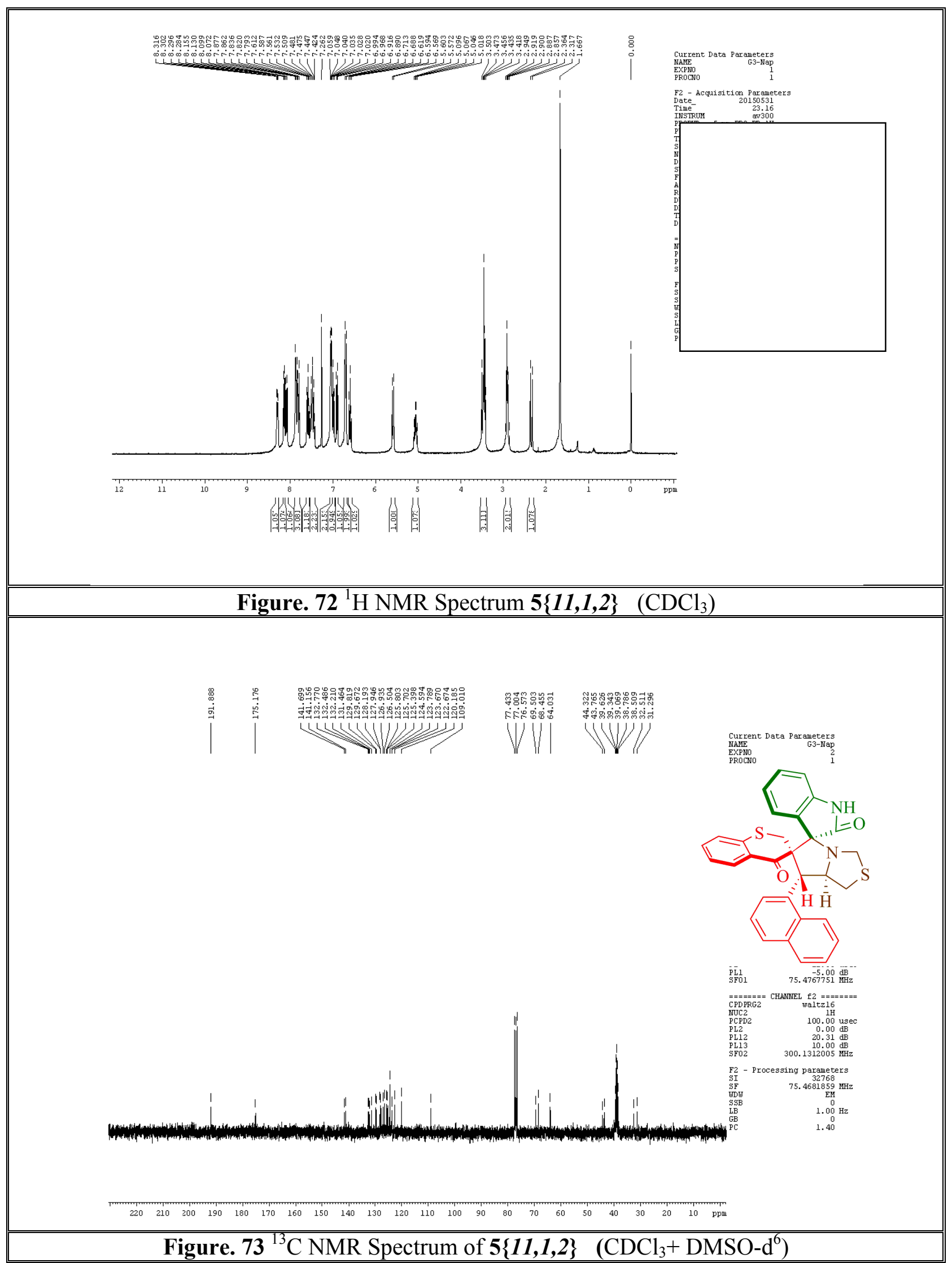




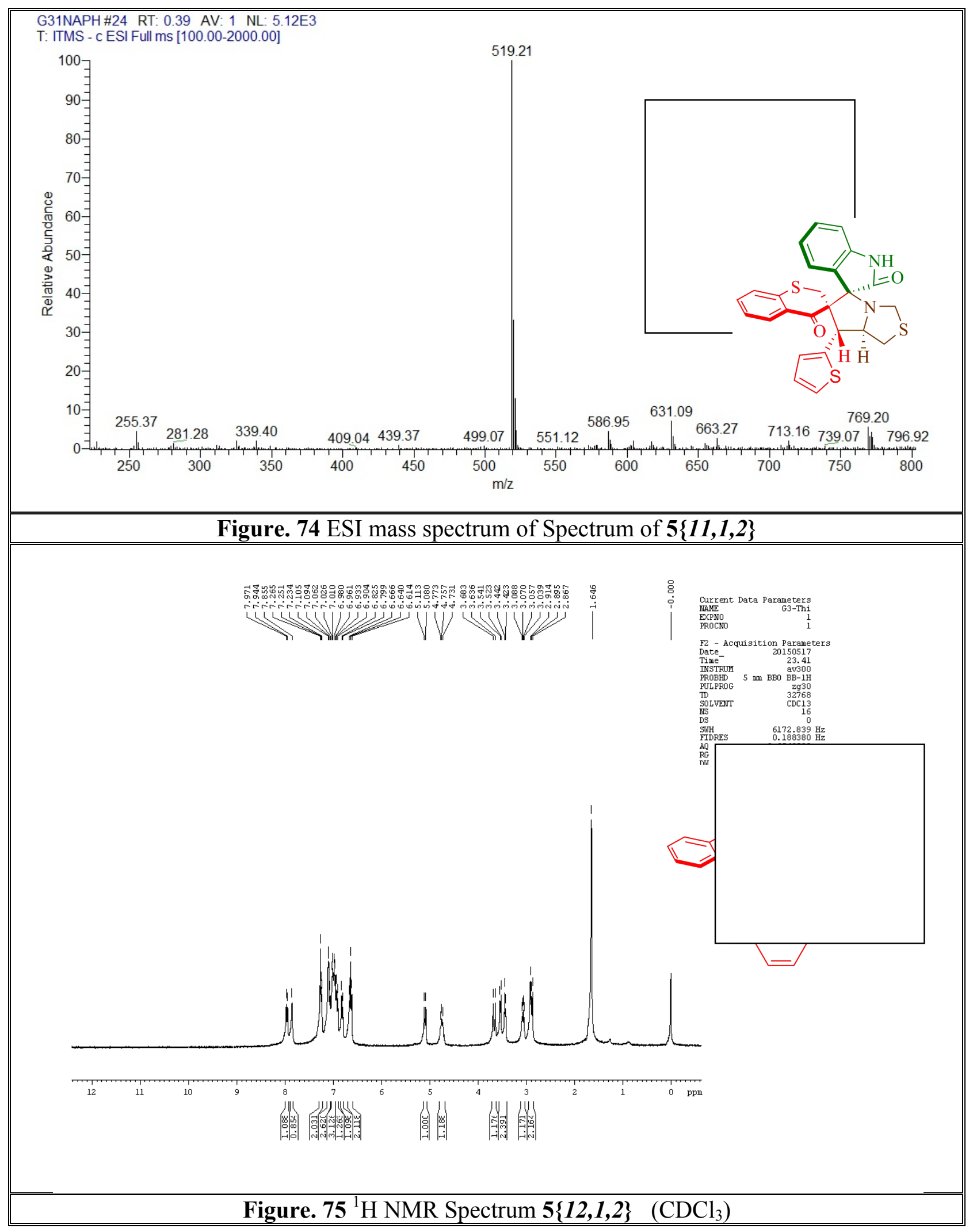




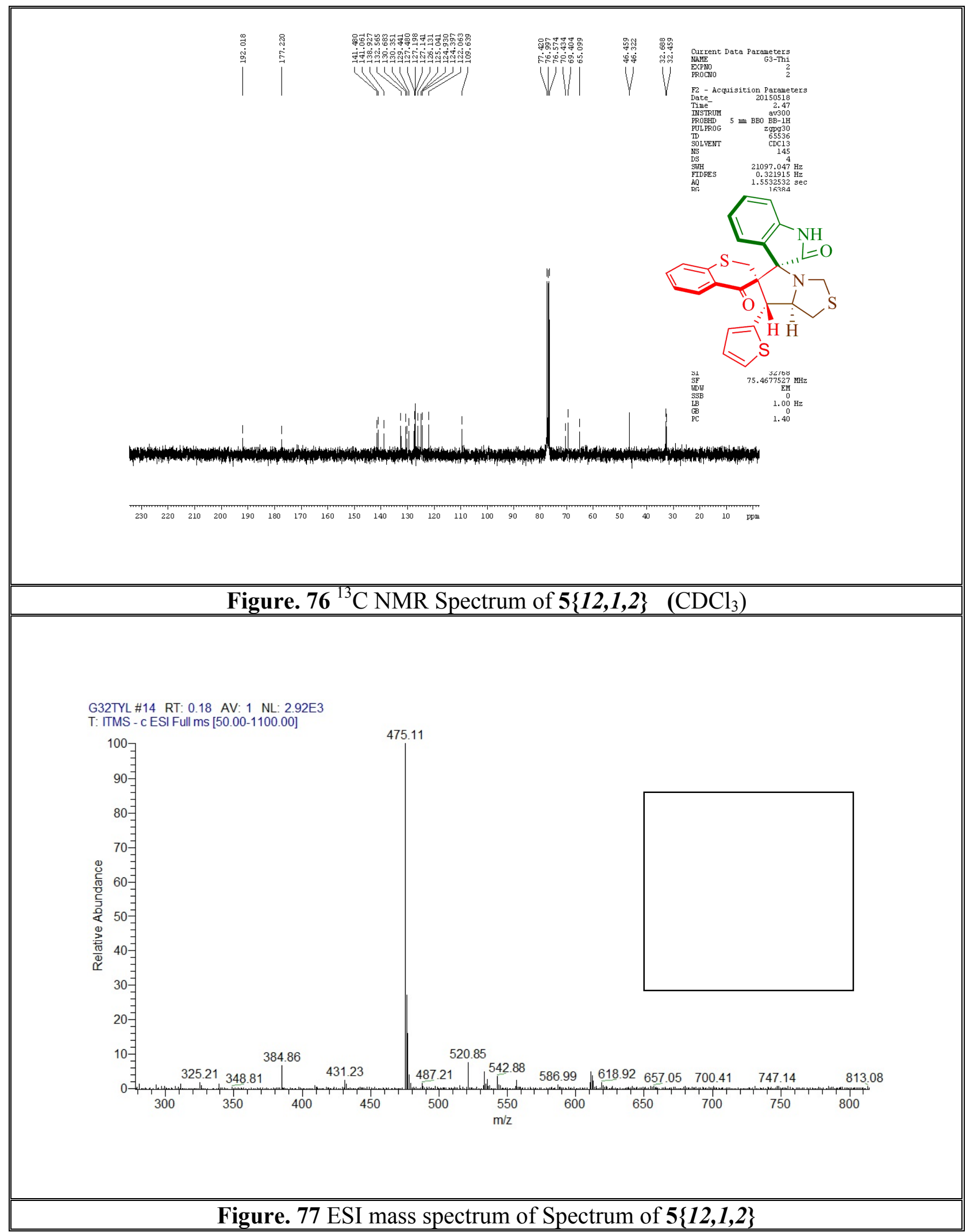




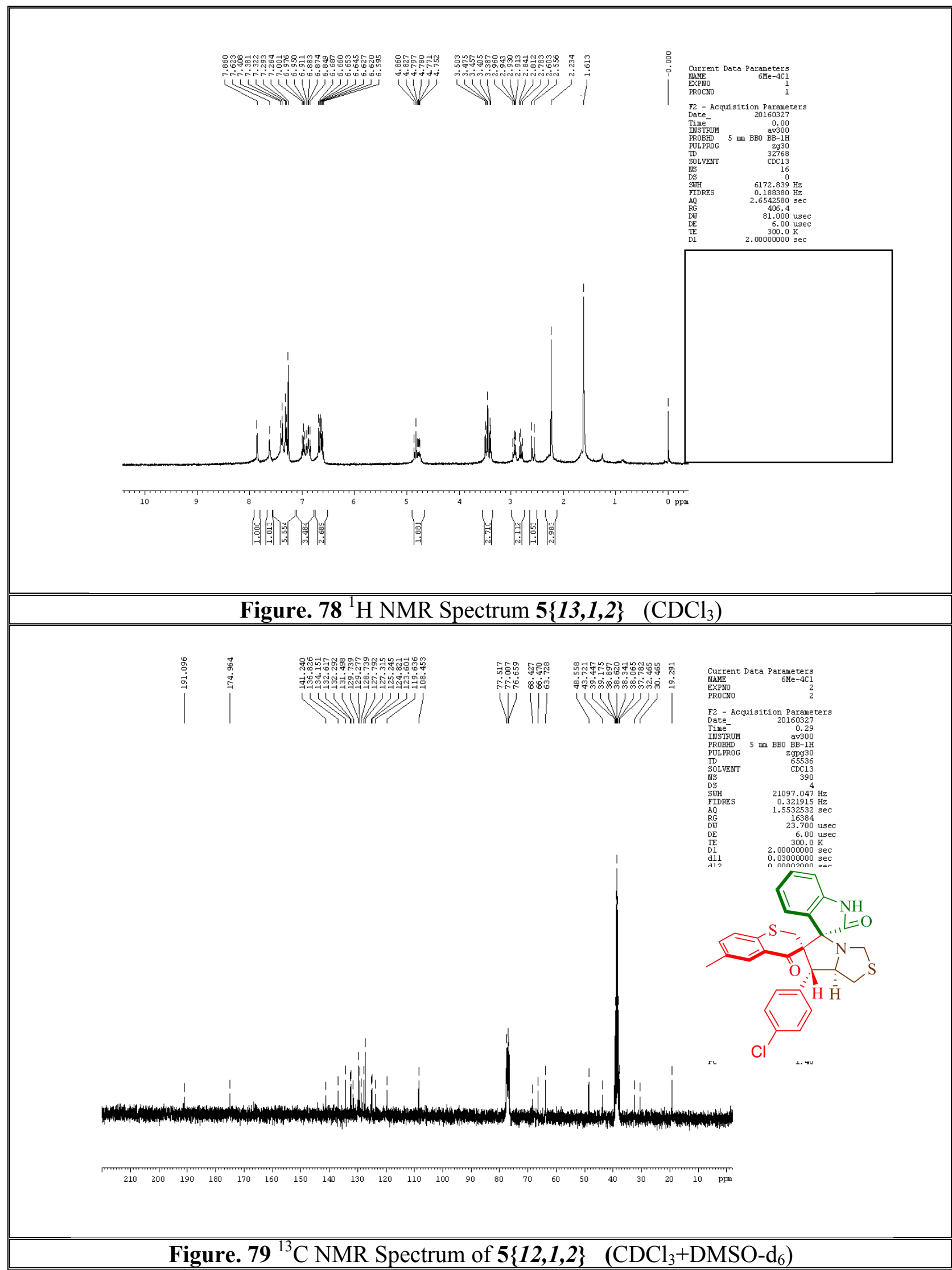




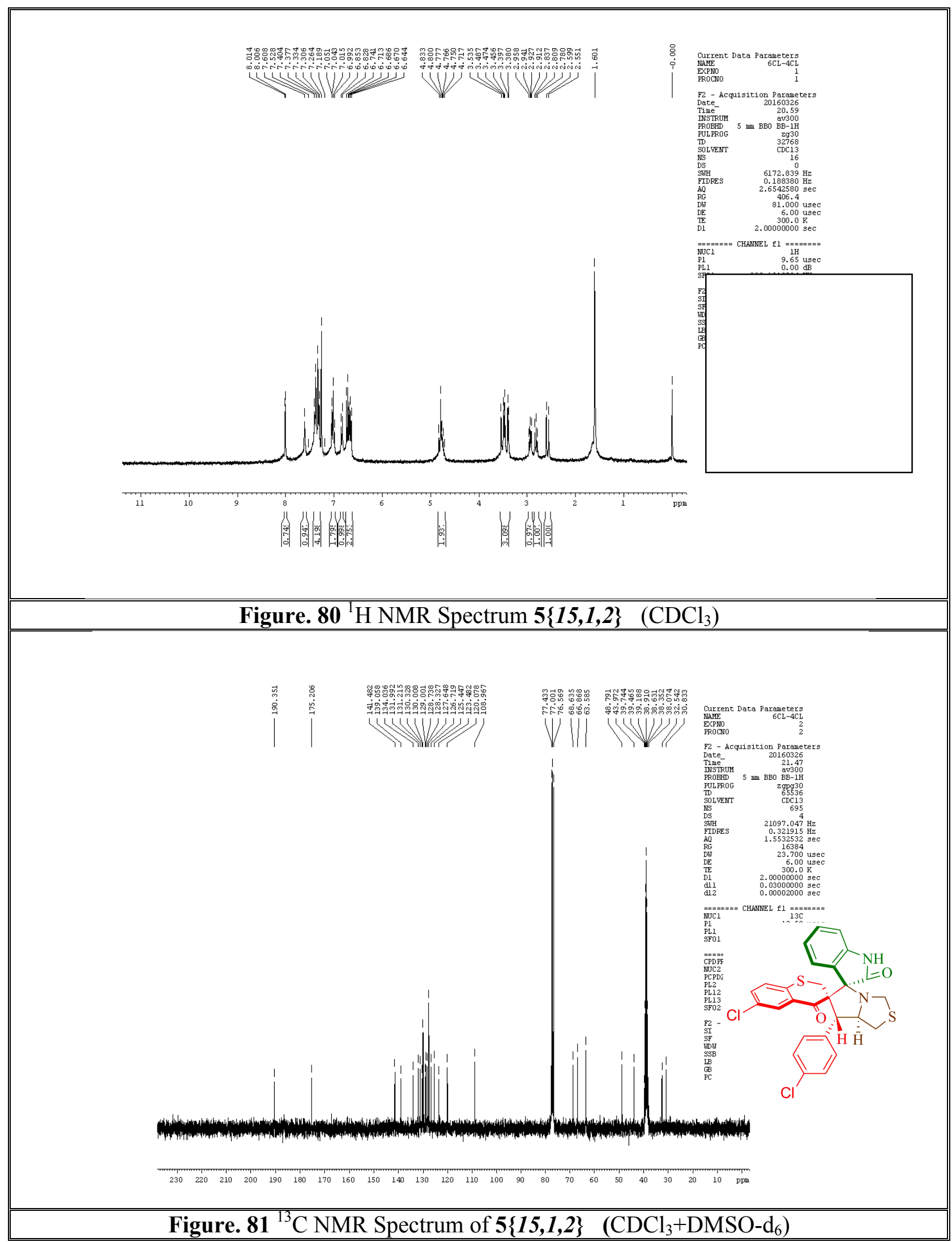




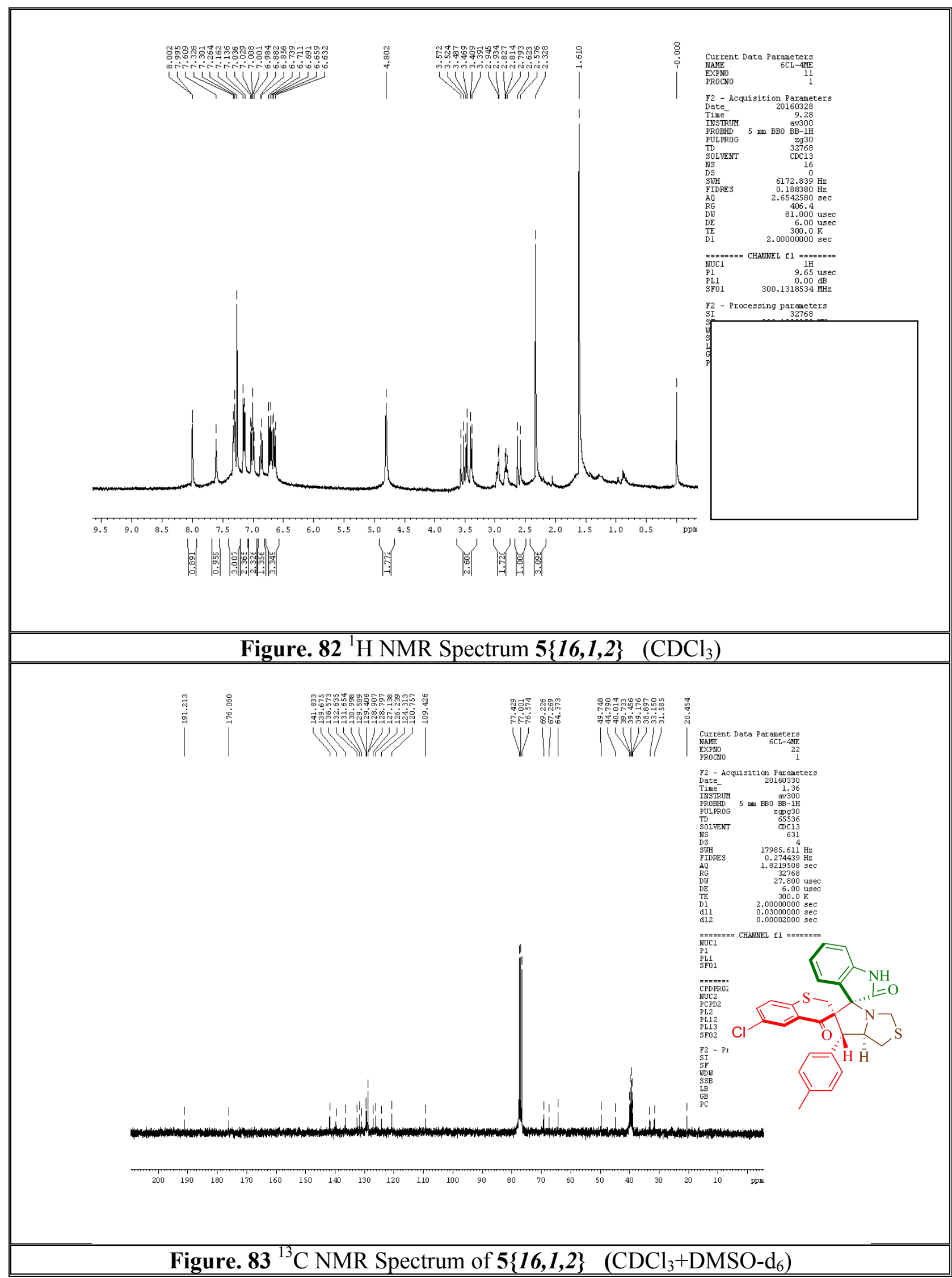




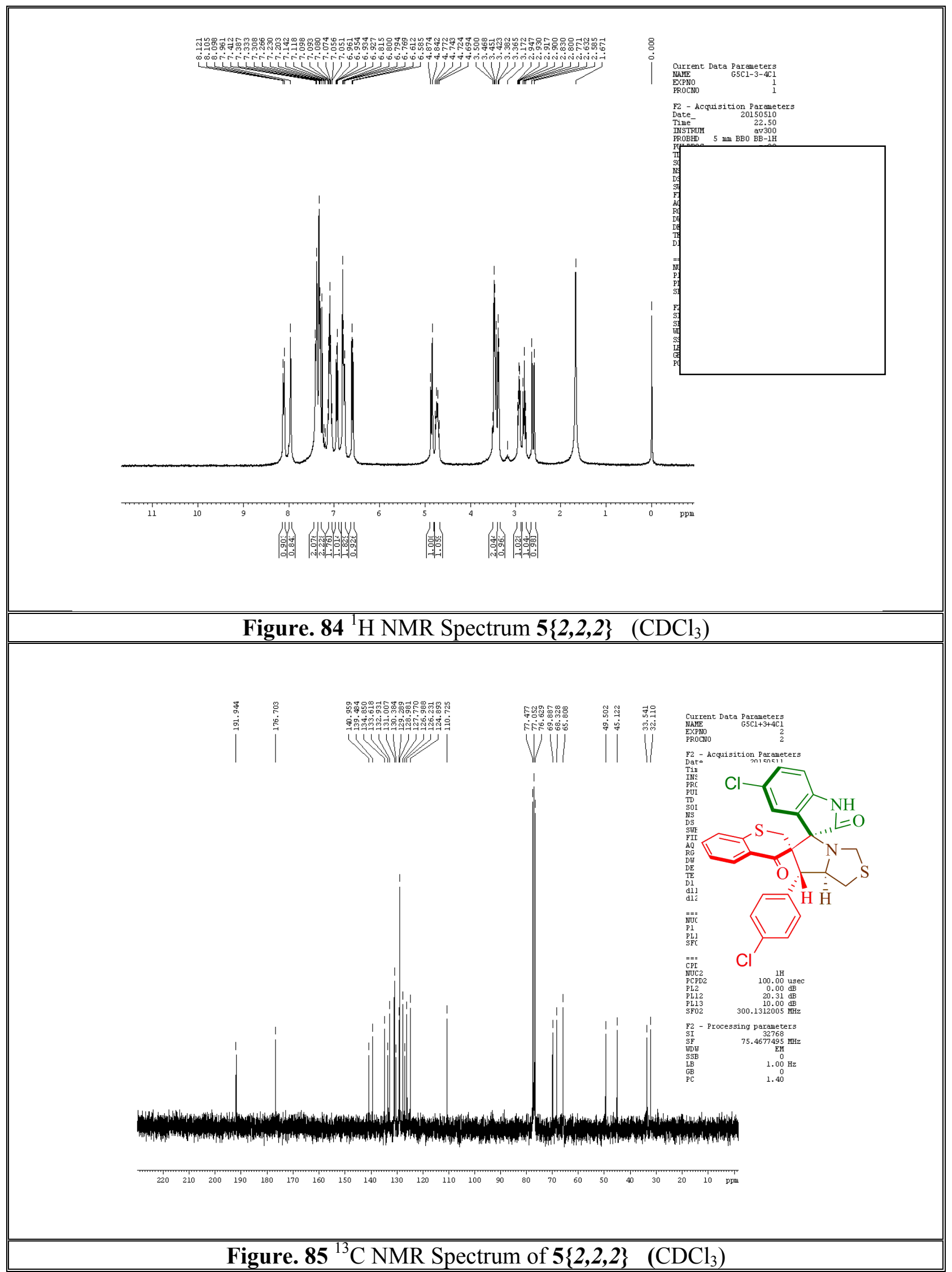




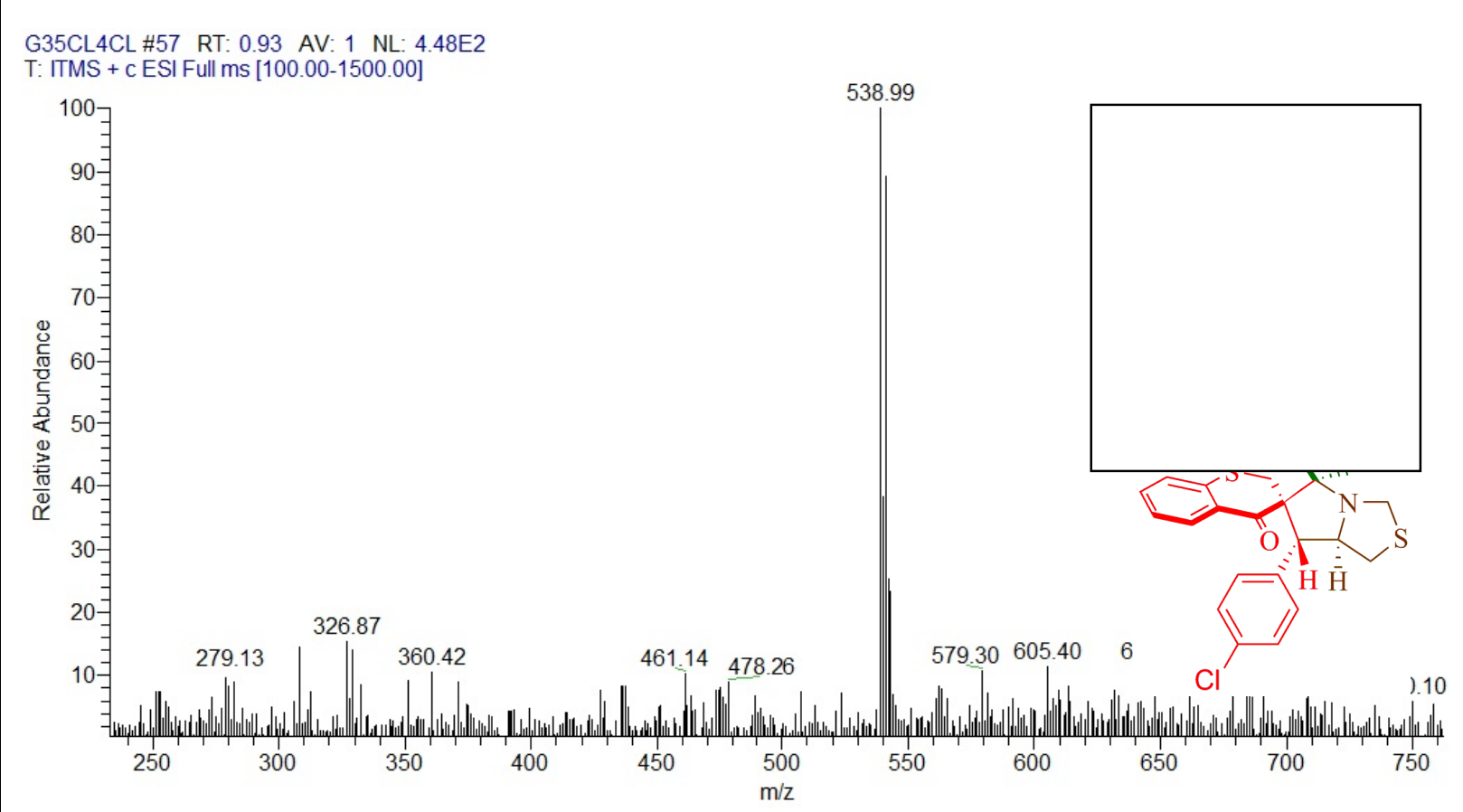

Figure. 86 ESI mass spectrum of Spectrum of 5\{2,2,2\}

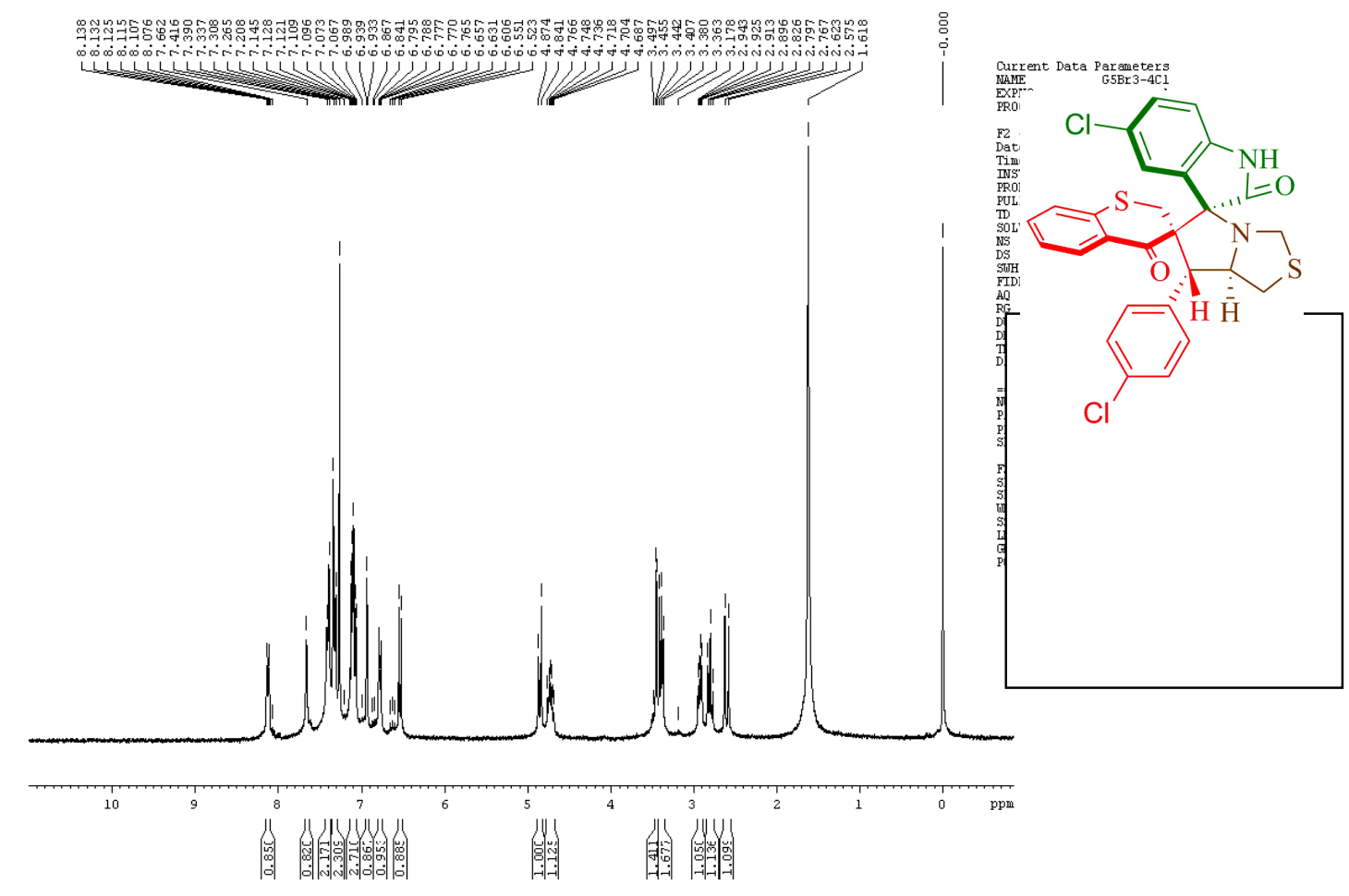

Figure. $87{ }^{1} \mathrm{H}$ NMR Spectrum 5\{2,3,2\} $\quad\left(\mathrm{CDCl}_{3}\right)$ 


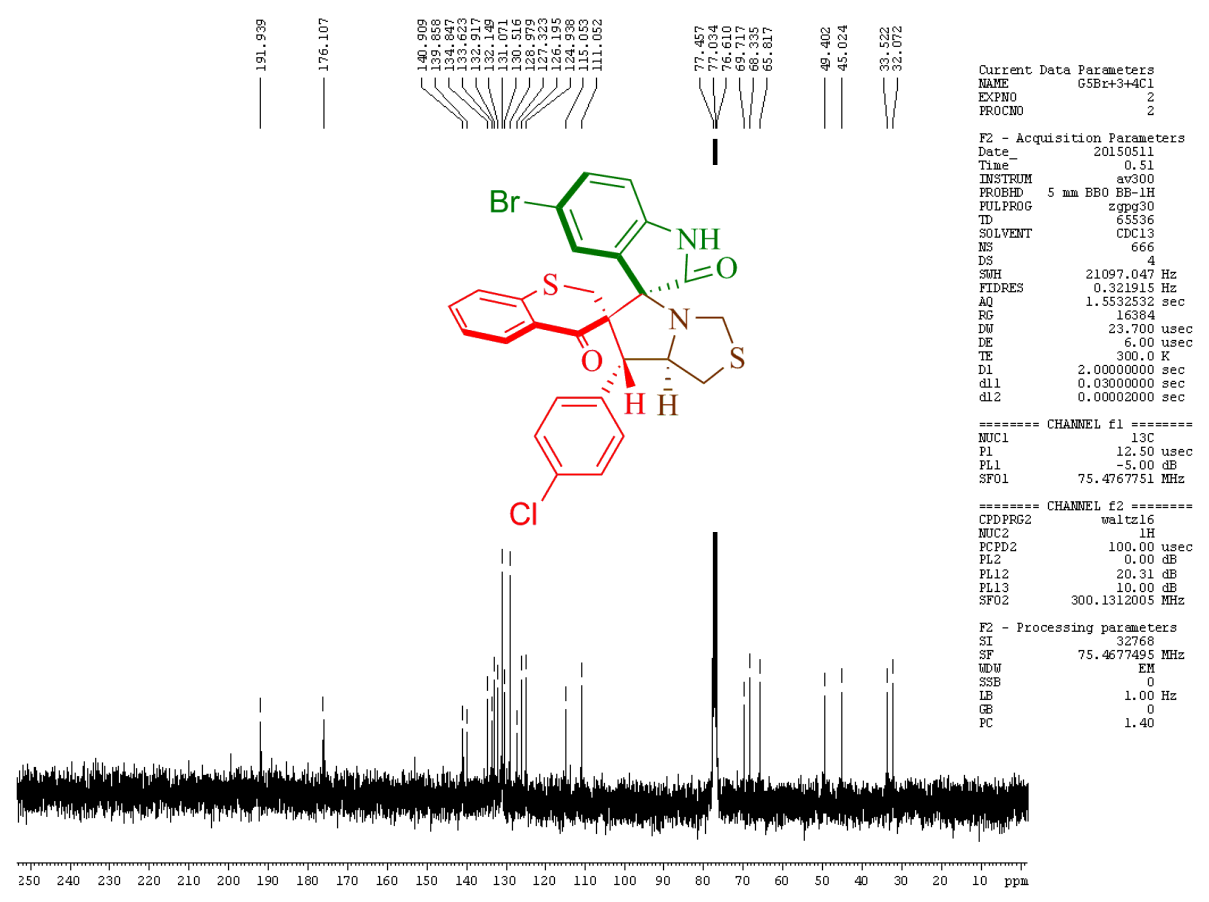

Figure. 88 ${ }^{13} \mathrm{C}$ NMR Spectrum of $5\{2,3,2\} \quad\left(\mathrm{CDCl}_{3}\right)$

G35BR4CL \#24 RT: 0.32 AV: 1 NL: 1.48E3

T: ITMS - c ESI Full ms [50.00-1100.00]

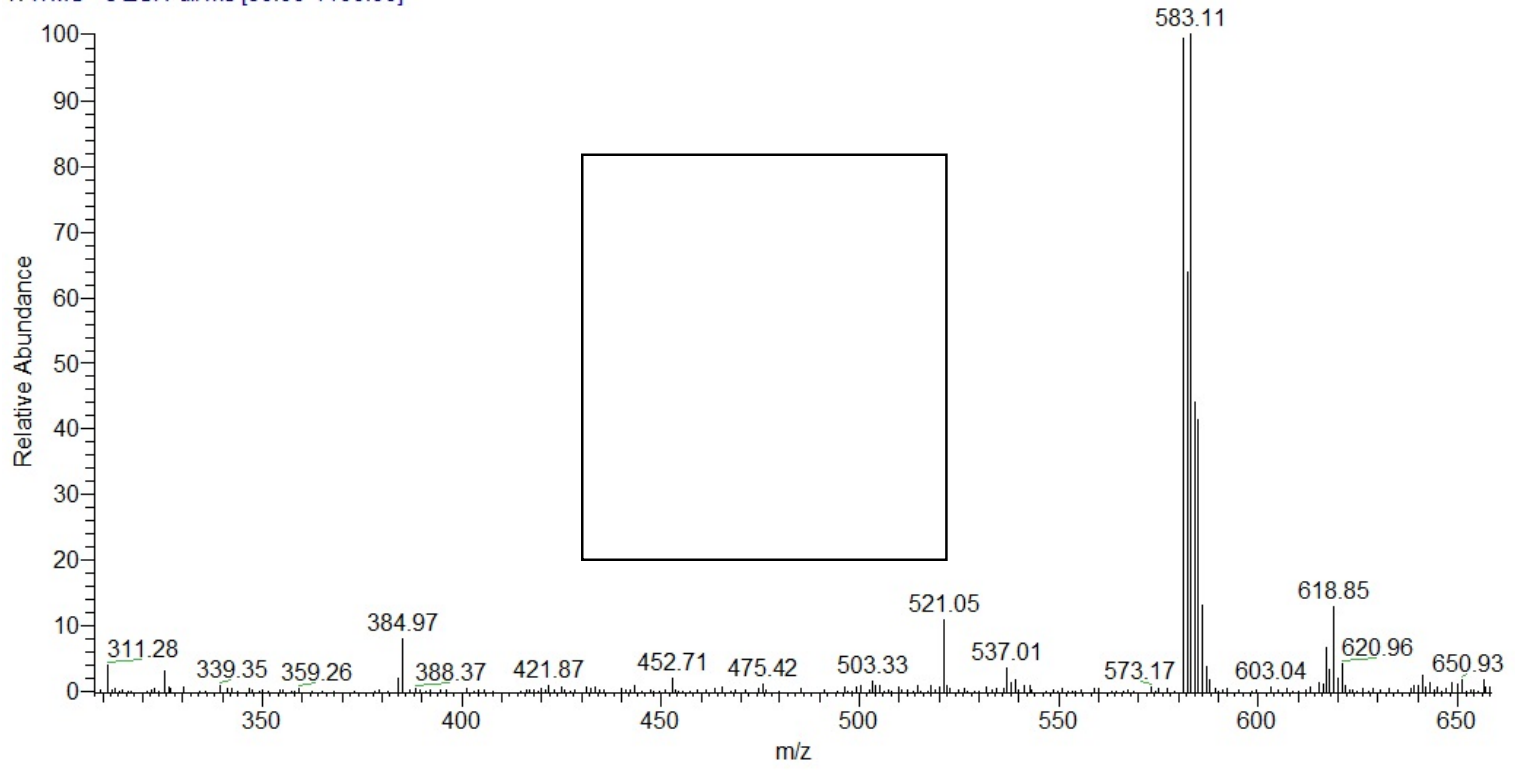

Figure. 89 ESI mass spectrum of Spectrum of $\mathbf{5}\{2,3,2\}$ 